\title{
Nonassociative differential geometry and gravity with non-geometric fluxes
}

\author{
Paolo Aschieri, ${ }^{a, b, c}$ Marija Dimitrijević Ćirić ${ }^{d}$ and Richard J. Szabo ${ }^{e, f, g}$ \\ ${ }^{a}$ Dipartimento di Scienze e Innovazione Tecnologica, Università del Piemonte Orientale, \\ Viale T. Michel 11, 15121 Alessandria, Italy \\ ${ }^{b}$ INFN, Sezione di Torino, \\ via P. Giuria 1, 10125 Torino, Italy \\ ${ }^{c}$ Arnold-Regge Center, \\ via P. Giuria 1, 10125, Torino, Italy \\ ${ }^{d}$ Faculty of Physics, University of Belgrade, \\ Studentski trg 12, 11000 Beograd, Serbia \\ ${ }^{e}$ Department of Mathematics, Heriot-Watt University, \\ Colin Maclaurin Building, Riccarton, Edinburgh EH14 4AS, U.K. \\ ${ }^{f}$ Maxwell Institute for Mathematical Sciences, \\ Edinburgh, U.K. \\ ${ }^{g}$ The Higgs Centre for Theoretical Physics, \\ Edinburgh, U.K. \\ E-mail: aschieri@to.infn.it, dmarija@ipb.ac.rs, R.J.Szabo@hw.ac.uk
}

ABSTRACT: We systematically develop the metric aspects of nonassociative differential geometry tailored to the parabolic phase space model of constant locally non-geometric closed string vacua, and use it to construct preliminary steps towards a nonassociative theory of gravity on spacetime. We obtain explicit expressions for the torsion, curvature, Ricci tensor and Levi-Civita connection in nonassociative Riemannian geometry on phase space, and write down Einstein field equations. We apply this formalism to construct $R$-flux corrections to the Ricci tensor on spacetime, and comment on the potential implications of these structures in non-geometric string theory and double field theory.

KEYwords: Non-Commutative Geometry, Flux compactifications, Models of Quantum Gravity

ARXIV EPRINT: 1710.11467 


\section{Contents}

1 Introduction $\quad 2$

2 Algebraic structure of non-geometric flux deformations 4

$2.1 R$-flux induced cochain twist and quasi-Hopf algebra 4

2.2 Associator identities 7

2.3 Double field theory formulation 8

3 Nonassociative deformation of tensor calculus $\quad 9$

3.1 Principles of twist deformation 9

$\begin{array}{llr}3.2 & \text { Functions } & 10\end{array}$

$\begin{array}{lll}3.3 & \text { Forms } & 11\end{array}$

$\begin{array}{lll}3.4 & \text { Tensors } & 12\end{array}$

3.5 Duality 13

3.6 Module homomorphisms 15

3.7 Quantum Lie algebra of diffeomorphisms 18

4 Nonassociative differential geometry 20

$\begin{array}{lll}4.1 \text { Connections } & 20\end{array}$

4.2 Dual connections 21

4.3 Connections on tensor products 23

4.4 Torsion 24

$\begin{array}{ll}4.5 & \text { Curvature }\end{array}$

4.6 Ricci tensor 32

5 Nonassociative Riemannian geometry and gravity 34

5.1 Metric and torsion-free connection conditions 34

5.2 Inversion in $A_{\star} \quad 36$

5.3 Levi-Civita connection 40

5.4 Einstein equations 44

5.5 Spacetime field equations 44

$\begin{array}{lll}5.6 & \text { First order corrections } & 46\end{array}$

6 Conclusions $\quad 50$ 


\section{Introduction}

Deformations of spacetime geometry through compactifications of string theory may help elucidate the precise mechanism by which closed strings provide a framework for a quantum theory of gravity. This has been the hope in some recent investigations surrounding non-geometric string theory, in which noncommutative and nonassociative deformations of target space geometry have been purported to be probed by closed strings in non-geometric flux compactifications $[1,9,13,15-17,19,25]$. In particular, in locally non-geometric backgrounds one aims to find a low-energy limit of closed string theory which is described by an effective nonassociative theory of gravity on spacetime.

In this paper we focus on the parabolic phase space model for strings propagating in locally non-geometric constant $R$-flux backgrounds in $d$ dimensions [25]. In this framework the canoncial commutation relations of phase space are deformed by a trivector $R$-flux on target space to the quasi-Poisson coordinate algebra

$$
\left[x^{\mu}, x^{\nu}\right]=\frac{\mathrm{i} \ell_{s}^{3}}{3 \hbar} R^{\mu \nu \rho} p_{\rho}, \quad\left[x^{\mu}, p_{\nu}\right]=\mathrm{i} \hbar \delta^{\mu}{ }_{\nu} \quad \text { and } \quad\left[p_{\mu}, p_{\nu}\right]=0,
$$

with the nonassociativity of spacetime captured by the non-vanishing Jacobiators

$$
\left[x^{\mu}, x^{\nu}, x^{\rho}\right]:=\left[x^{\mu},\left[x^{\nu}, x^{\rho}\right]\right]+\left[x^{\nu},\left[x^{\rho}, x^{\mu}\right]\right]+\left[x^{\rho},\left[x^{\mu}, x^{\nu}\right]\right]=\ell_{s}^{3} R^{\mu \nu \rho} .
$$

On fields these deformations are described by nonassociative phase space starproducts $[8,24,26]$, and using them the formal mathematical development of nonassociative differential geometry of phase space has been pursued in $[5,10,11,27]$; a more pedestrian approach to these developments is given in $[12,14]$. The purpose of the present paper is two-fold.

Firstly, we present a self-contained construction of nonassociative differential geometry based on the constant parabolic $R$-flux background which is rooted in two guiding principles: equivariance under the twist deformed quasi-Hopf algebra of infinitesimal diffeomorphisms on the one hand (including invariance of the operations of multiplication, inner derivation and exterior derivation of fields, and covariance of tensor fields) and, on the other hand, the equivalent descriptions of tensor fields as sections of vector bundles and as maps between vector bundles. These constructions are compatible with the category theory formalism of $[10,11]$; indeed, sections $2-4$ of the present paper can also be regarded as unravelling that general construction for the specific cochain twist deformation provided by the constant parabolic $R$-flux model in phase space. This unravelling complements that undertaken in [12]. However, as pursued by [14], the main viewpoint here is to avoid the use of category theory altogether and yet, in contrast to the more pedestrian approach of [14], to provide a self-consistent and mathematically rigorous construction of nonassociative differential geometry. This leads to a notion of torsion that coincides with that introduced in [14] (see (4.35)). On the other hand, it leads to key new results, including a simple definition of curvature tensor as the square of the covariant derivative, its equivalent description as an operator on vector fields (the second Cartan structure equation, see (4.55)), and a well-defined Ricci tensor (see (4.75) and (4.83)). 
Secondly, we use this framework to systematically study the metric aspects of nonassociative differential geometry. This is a nonassociative generalization of the noncommutative Riemannian geometry developed in $[6,7]$. One of our main achievements is the construction of the analog of the Levi-Civita connection (see (5.49)), wherein we describe how to circumvent the problems encountered in [14]. We thus obtain a metric formulation of nonassociative gravity on phase space. A complementary vielbein or first order formalism for nonassociative gravity has been considered in $[11,12]$. Here we have chosen to develop the metric aspects of nonassociative gravity, because it also represents the most direct way to explore the potential relevance of nonassociative gravity to string theory, in that in closed string theory the fundamental field is the metric tensor rather than the vielbein.

Although it is interesting in its own right to be able to implement general covariance under the quasi-Hopf algebra of deformed diffeomorphisms and to formulate Einstein equations in nonassociative space, in order to arrive at a theory that can be potentially considered as providing a low-energy effective action for closed strings in the presence of non-geometric fluxes, it is necessary to project nonassociative gravity from phase space to spacetime. To this aim we further develop the approach of [5], which demonstrated how to extract results of closed string scattering amplitudes in non-geometric backgrounds [16] from the nonassociative deformation of phase space. We conclude in particular that, in the constant parabolic $R$-flux model, the curvature of spacetime is deformed in a non-trivial way by locally non-geometric fluxes. Our main result for the Ricci tensor of nonassociative gravity is presented in (5.90) and reproduced here:

$$
\begin{aligned}
\operatorname{Ric}_{\mu \nu}^{\circ}= & \operatorname{Ric}_{\mu \nu}^{\mathrm{LC}}+\frac{\ell_{s}^{3}}{12} R^{\alpha \beta \gamma}\left(\partial_{\rho}\left(\partial_{\alpha} \mathrm{g}^{\rho \sigma}\left(\partial_{\beta} \mathrm{g}_{\sigma \tau}\right) \partial_{\gamma} \Gamma_{\mu \nu}^{\mathrm{LC} \tau}\right)-\partial_{\nu}\left(\partial_{\alpha} \mathrm{g}^{\rho \sigma}\left(\partial_{\beta} \mathrm{g}_{\sigma \tau}\right) \partial_{\gamma} \Gamma_{\mu \rho}^{\mathrm{LC} \tau}\right)\right. \\
& +\partial_{\gamma} \mathrm{g}_{\tau \omega}\left(\partial_{\alpha}\left(\mathrm{g}^{\sigma \tau} \Gamma_{\sigma \nu}^{\mathrm{LC} \rho}\right) \partial_{\beta} \Gamma_{\mu \rho}^{\mathrm{LC} \omega}-\partial_{\alpha}\left(\mathrm{g}^{\sigma \tau} \Gamma_{\sigma \rho}^{\mathrm{LC} \rho}\right) \partial_{\beta} \Gamma_{\mu \nu}^{\mathrm{LC} \omega}\right. \\
& +\left(\Gamma_{\mu \rho}^{\mathrm{LC} \sigma} \partial_{\alpha} \mathrm{g}^{\rho \tau}-\partial_{\alpha} \Gamma_{\mu \rho}^{\mathrm{LC} \sigma} \mathrm{g}^{\rho \tau}\right) \partial_{\beta} \Gamma_{\sigma \nu}^{\mathrm{LC} \omega} \\
& \left.\left.-\left(\Gamma_{\mu \nu}^{\mathrm{LC} \sigma} \partial_{\alpha} \mathrm{g}^{\rho \tau}-\partial_{\alpha} \Gamma_{\mu \nu}^{\mathrm{LC} \sigma} \mathrm{g}^{\rho \tau}\right) \partial_{\beta} \Gamma_{\sigma \rho}^{\mathrm{LC} \omega}\right)\right)
\end{aligned}
$$

where $\mathrm{Ric}_{\mu \nu}^{\mathrm{LC}}$ is the usual Ricci tensor of the classical Levi-Civita connection $\Gamma_{\mu \nu}^{\mathrm{LC} \rho}$ of a metric tensor $\mathrm{g}_{\mu \nu}$ on spacetime. This expression is valid to linear order in the $R$-flux, which is the order at which the corresponding conformal field theory calculations are reliable [16]. It represents the first non-trivial starting point for understanding how to define a nonassociative theory of gravity describing the low-energy effective dynamics of closed strings in non-geometric backgrounds, although in this paper we do not address in detail the implications of this structure on string theory or double field theory [17]; see [14] for some discussion of points which should be addressed in this latter context.

A somewhat perplexing aspect about the development of the geometry of the phase space model for the $R$-flux background concerns the precise meaning of Riemannian geometry of phase space. Superficially, our approach is reminescent of recent discussions of Born geometry in string theory [21], wherein it is argued that the fundamental string symmetries should contain diffeomorphisms of phase space, in accord with Born's original proposal to unify quantum theory with general relativity by treating spacetime and momentum space on equal footing. It is precisely the phase space formulation that is responsible for nonas- 
sociativity in the parabolic $R$-flux model [5, 26, 27], and it would be interesting to find explicit connections between our constructions and the proposal of [21].

The outline of the remainder of this paper is as follows. In section 2 we describe some preliminary Hopf algebraic ingredients and define the quasi-Hopf algebra of infinitesimal diffeomorphisms. This is the symmetry algebra that leads us into the nonassociative deformations of differential geometry, and in this preliminary section we follow [5, 10, 27], where further details can be found; we also comment on how our constructions fit into frameworks suitable for a double field theory formulation of all our developments. In section 3 we use these ingredients to fully develop nonassociative tensor calculus on phase space, and apply it in section 4 to construct a nonassociative theory of connections, obtaining new definitions of curvature and Ricci tensors, together with the main results of the Cartan structure equations for curvature and torsion. This section builds and expands on the nonassociative differential geometry machinery developed by [11], and on the noncommutative geometry techniques and results of $[2,6]$. In section 5 we introduce metric tensors and develop the Riemannian aspects of nonassociative differential geometry, including the extension to the nonassociative setting of the noncommutative metric compatibility condition $[2,7]$, the explicit construction of the Levi-Civita connection, the corresponding Ricci tensor and vacuum Einstein equations on nonassociative phase space, and the induced corrections to the spacetime Ricci tensor given in (1.3). Finally, in section 6 we conclude by summarising our main findings and highlighting key open issues for further investigation.

\section{Algebraic structure of non-geometric flux deformations}

\section{$2.1 \quad R$-flux induced cochain twist and quasi-Hopf algebra}

The presence of a constant non-geometric $R$-flux on $M=\mathbb{R}^{d}$ has been proposed to be captured by a certain nonassociative deformation of the geometry of phase space $\mathcal{M}=$ $T^{*} M=\mathbb{R}^{d} \times\left(\mathbb{R}^{d}\right)^{*}$. Coordinates on $\mathcal{M}$ will be denoted $x^{A}=\left(x^{\mu}, \tilde{x}_{\mu}\right)$ with $A=1, \ldots, 2 d$, where $x^{\mu}$ are spacetime coordinates on $M$ while $\tilde{x}_{\mu}=p_{\mu}$ are momentum coordinates for $\mu=1, \ldots, d$. Derivatives are denoted in a similar way: $\partial_{A}=\left(\frac{\partial}{\partial x^{\mu}}=\partial_{\mu}, \frac{\partial}{\partial p_{\mu}}=\tilde{\partial}^{\mu}\right)$. In string theory applications, the metric on $M$ is usually taken to have Euclidean signature, but in what follows our results do not depend on the signature of the spacetime metric tensor which can be either Euclidean or Lorentzian.

The geometry of phase space $\mathcal{M}$ is deformed by using a particular cochain twist element $\mathcal{F}$ in the universal enveloping Hopf algebra $U \operatorname{Vec}(\mathcal{M})$ of the Lie algebra of vector fields $\operatorname{Vec}(\mathcal{M})$ on $\mathcal{M}$. It is defined by

$$
\mathcal{F}=\exp \left(-\frac{\mathrm{i} \hbar}{2}\left(\partial_{\mu} \otimes \tilde{\partial}^{\mu}-\tilde{\partial}^{\mu} \otimes \partial_{\mu}\right)-\frac{\mathrm{i} \ell_{s}^{3}}{12 \hbar} R^{\mu \nu \rho}\left(p_{\nu} \partial_{\rho} \otimes \partial_{\mu}-\partial_{\mu} \otimes p_{\nu} \partial_{\rho}\right)\right),
$$

where $R^{\mu \nu \rho}$ are the totally antisymmetric constant $R$-flux components, and implicit summation over repeated upper and lower indices is always understood. We will write

$$
\mathcal{F}=: \mathrm{f}^{\alpha} \otimes \mathrm{f}_{\alpha}=1 \otimes 1+O\left(\hbar, \ell_{s}^{3}\right)
$$


where $\mathrm{f}^{\alpha}, \mathrm{f}_{\alpha}$ are elements in $U \operatorname{Vec}(\mathcal{M})$ and summation on $\alpha$ is understood; for the inverse of the twist we write $\mathcal{F}^{-1}=: \overline{\mathrm{f}}^{\alpha} \otimes \overline{\mathrm{f}}_{\alpha}$. Following [5, 27], it will sometimes be convenient to regard the twist element (2.1) as the result of applying successively two commuting abelian cocycle twists

$$
\mathcal{F}=F F_{R}=F_{R} F
$$

where the Hopf 2-cocycle

$$
F=\exp \left(-\frac{\mathrm{i} \hbar}{2}\left(\partial_{\mu} \otimes \tilde{\partial}^{\mu}-\tilde{\partial}^{\mu} \otimes \partial_{\mu}\right)\right)=: \mathbf{f}^{\alpha} \otimes \mathbf{f}_{\alpha}=1 \otimes 1+O(\hbar)
$$

implements the standard Moyal-Weyl deformation of canonical phase space, while the 2-cocycle

$$
F_{R}=\exp \left(-\frac{\mathrm{i} \kappa}{2} R^{\mu \nu \rho}\left(p_{\nu} \partial_{\rho} \otimes \partial_{\mu}-\partial_{\mu} \otimes p_{\nu} \partial_{\rho}\right)\right)=: \mathrm{f}_{R}^{\alpha} \otimes \mathrm{f}_{R \alpha}=1 \otimes 1+O(\kappa),
$$

with

$$
\kappa:=\frac{\ell_{s}^{3}}{6 \hbar},
$$

implements the deformation by the $R$-flux; in the following we shall sometimes treat $\hbar$ and $\kappa$ as independent (small) deformation parameters.

The Hopf algebra $U \operatorname{Vec}(\mathcal{M})$ has coproduct $\Delta$ defined as $\Delta(1)=1 \otimes 1, \Delta\left(\partial_{A}\right)=$ $1 \otimes \partial_{A}+\partial_{A} \otimes 1$, counit $\epsilon$ defined as $\epsilon(1)=1, \epsilon\left(\partial_{A}\right)=0$, and antipode $S$ defined as $S(1)=1$, $S\left(\partial_{A}\right)=-\partial_{A}$, with $\Delta$ and $\epsilon$ extended to all of $U \operatorname{Vec}(\mathcal{M})$ as algebra homomorphisms, and $S$ extended as an algebra antihomomorphism (linear and anti-multiplicative). With the twist $\mathcal{F}$, following [27] we deform the Hopf algebra $U \operatorname{Vec}(\mathcal{M})$ (considered to be extended with power series in $\hbar$ and $\kappa$ ) to the quasi-Hopf algebra $U \operatorname{Vec}^{\mathcal{F}}(\mathcal{M})$. It has the same algebra structure as $U \operatorname{Vec}(\mathcal{M})$ and coproduct $\Delta_{\mathcal{F}}=\mathcal{F} \Delta \mathcal{F}^{-1}$; explicitly, on the basis vector fields we have

$$
\begin{aligned}
& \Delta_{\mathcal{F}}\left(\partial_{\mu}\right)=1 \otimes \partial_{\mu}+\partial_{\mu} \otimes 1, \\
& \Delta_{\mathcal{F}}\left(\tilde{\partial}^{\mu}\right)=1 \otimes \tilde{\partial}^{\mu}+\tilde{\partial}^{\mu} \otimes 1+\mathrm{i} \kappa R^{\mu \nu \rho} \partial_{\nu} \otimes \partial_{\rho} .
\end{aligned}
$$

The quasi-antipode is $S_{\mathcal{F}}=S$, where the quasi-antipode elements $\alpha_{\mathcal{F}}$ and $\beta_{\mathcal{F}}$ are the identity in the case of the twist $\mathcal{F}$, because $\alpha=\beta=1$ in $U \operatorname{Vec}(\mathcal{M})$ and

$$
\mathrm{f}^{\alpha} S\left(\mathbf{f}_{\alpha}\right)=\mathbf{f}_{R}^{\alpha} S\left(\mathbf{f}_{R \alpha}\right)=\overline{\mathbf{f}}^{\alpha} S\left(\overline{\mathbf{f}}_{\alpha}\right)=\overline{\mathbf{f}}_{R}^{\alpha} S\left(\overline{\mathbf{f}}_{R \alpha}\right)=1
$$

(see [27], section 2.2). The counit is $\epsilon_{\mathcal{F}}=\epsilon$. The twist $\mathcal{F}$ does not fulfill the 2-cocycle condition, and instead one obtains

$$
\Phi(\mathcal{F} \otimes 1)(\Delta \otimes \mathrm{id}) \mathcal{F}=(1 \otimes \mathcal{F})(\mathrm{id} \otimes \Delta) \mathcal{F}
$$

where the element $\Phi$, called the associator, is the Hopf 3-cocycle

$$
\Phi=\exp \left(\frac{\ell_{s}^{3}}{6} R^{\mu \nu \rho} \partial_{\mu} \otimes \partial_{\nu} \otimes \partial_{\rho}\right)=: \phi_{1} \otimes \phi_{2} \otimes \phi_{3}=1 \otimes 1 \otimes 1+O\left(\ell_{s}^{3}\right)
$$


with summation understood in the second expression (as in e.g. $\mathcal{F}=\mathrm{f}^{\alpha} \otimes \mathrm{f}_{\alpha}$ ). The inverse associator is denoted $\Phi^{-1}=: \bar{\phi}_{1} \otimes \bar{\phi}_{2} \otimes \bar{\phi}_{3}$. The failure of the 2-cocycle condition implies that the twisted coproduct $\Delta_{\mathcal{F}}$ is no longer coassociative, as one sees from the quasi-coassociativity relation

$$
\Phi\left(\Delta_{\mathcal{F}} \otimes \mathrm{id}\right) \Delta_{\mathcal{F}}(\xi)=\left(\mathrm{id} \otimes \Delta_{\mathcal{F}}\right) \Delta_{\mathcal{F}}(\xi) \Phi
$$

for all $\xi \in U \operatorname{Vec}(\mathcal{M})$.

The sextuple $\left(U \operatorname{Vec}(\mathcal{M}), \cdot, \Delta_{\mathcal{F}}, \Phi, S, \epsilon\right)$ defines on the vector space $U \operatorname{Vec}(\mathcal{M})$ the structure of a quasi-Hopf algebra $U \operatorname{Vec}^{\mathcal{F}}(\mathcal{M})[20]$. In $U \operatorname{Vec}^{\mathcal{F}}(\mathcal{M})$ the only relaxation of the Hopf algebra structure is the presence of a non-trivial associator $\Phi$ for the coproduct $\Delta_{\mathcal{F}}$. The quasi-Hopf algebra $U \operatorname{Vec}^{\mathcal{F}}(\mathcal{M})$ will play the role of the symmetry algebra of infinitesimal diffeomorphisms of the nonassociative deformation of phase space $\mathcal{M}$.

For later use, we rewrite the relation (2.11), which expresses the failure of coassociativity of $\Delta_{\mathcal{F}}$, in the form

$$
\left(\xi_{(1)_{(1)}} \otimes \xi_{(1)_{(2)}} \otimes \xi_{(2)}\right) \Phi^{-1}=\Phi^{-1}\left(\xi_{(1)} \otimes \xi_{(2)_{(1)}} \otimes \xi_{(2)_{(2)}}\right) .
$$

Here we introduced the Sweedler notation

$$
\Delta_{\mathcal{F}}(\xi)=: \xi_{(1)} \otimes \xi_{(2)}
$$

for the coproduct (with implicit summation) and its iterations, for example

$$
\left(\Delta_{\mathcal{F}} \otimes \mathrm{id}\right) \Delta_{\mathcal{F}}(\xi)=: \xi_{(1)_{(1)}} \otimes \xi_{(1)_{(2)}} \otimes \xi_{(2)} .
$$

Recalling that the quasi-antipode is just the undeformed antipode $S$, we also observe that its compatibility with the coproduct $\Delta_{\mathcal{F}}$,

$$
\xi_{(1)} S\left(\xi_{(2)}\right)=\epsilon(\xi)=S\left(\xi_{(1)}\right) \xi_{(2)},
$$

for all $\xi \in U \operatorname{Vec}^{\mathcal{F}}(\mathcal{M})$, follows from the equalities (2.8).

A further relevant property of the quasi-Hopf algebra $U \operatorname{Vec}^{\mathcal{F}}(\mathcal{M})$ is its triangularity. We denote by $\Delta_{\mathcal{F}}^{\text {op }}$ the opposite coproduct, obtained by flipping the two legs of $\Delta_{\mathcal{F}}$, i.e., if $\Delta_{\mathcal{F}}(\xi)=\xi_{(1)} \otimes \xi_{(2)}$ then $\Delta_{\mathcal{F}}^{\text {op }}(\xi):=\xi_{(2)} \otimes \xi_{(1)}$, for all $\xi \in U \operatorname{Vec}^{\mathcal{F}}(\mathcal{M})$. Since the coproduct of $U \operatorname{Vec}(\mathcal{M})$ satisfies $\Delta=\Delta^{\mathrm{op}}$, it follows immediately that the coproduct $\Delta_{\mathcal{F}}$ of $U \operatorname{Vec}^{\mathcal{F}}(\mathcal{M})$ satisfies the property $\Delta_{\mathcal{F}}^{\mathrm{op}}(\xi)=\mathcal{R} \Delta_{\mathcal{F}}(\xi) \mathcal{R}^{-1}$, for all $\xi \in U \operatorname{Vec}^{\mathcal{F}}(\mathcal{M})$, with the $\mathcal{R}$-matrix given by

$$
\mathcal{R}=\mathcal{F}^{-2}=: \mathrm{R}^{\alpha} \otimes \mathrm{R}_{\alpha} .
$$

Its inverse $\mathcal{R}^{-1}=\mathcal{F}^{2}=: \overline{\mathrm{R}}^{\alpha} \otimes \overline{\mathrm{R}}_{\alpha}$ satisfies

$$
\overline{\mathrm{R}}^{\alpha} \otimes \overline{\mathrm{R}}_{\alpha}=\mathrm{R}_{\alpha} \otimes \mathrm{R}^{\alpha}
$$

so that the $\mathcal{R}$-matrix is triangular. The quasi-Hopf algebra $U \operatorname{Vec}^{\mathcal{F}}(\mathcal{M})$ with this $\mathcal{R}$-matrix is a triangular quasi-Hopf algebra [10,22]. The coproduct of the inverse of the $\mathcal{R}$-matrix can be explicitly computed and reads

$$
\begin{aligned}
& \left(\Delta_{\mathcal{F}} \otimes \mathrm{id}\right) \mathcal{R}^{-1}=\Phi^{-3} \overline{\mathrm{R}}^{\beta} \otimes \overline{\mathrm{R}}^{\alpha} \otimes \overline{\mathrm{R}}_{\alpha} \overline{\mathrm{R}}_{\beta}, \\
& \left(\mathrm{id} \otimes \Delta_{\mathcal{F}}\right) \mathcal{R}^{-1}=\Phi^{3} \overline{\mathrm{R}}^{\alpha} \overline{\mathrm{R}}^{\beta} \otimes \overline{\mathrm{R}}_{\alpha} \otimes \overline{\mathrm{R}}_{\beta} .
\end{aligned}
$$


These equalities are a simplified version of the compatibility conditions between the coproduct $\Delta_{\mathcal{F}}$ and the $\mathcal{R}$-matrix that is due to the antisymmetry of the trivector $R^{\mu \nu \rho}$ (see (2.20) below).

\section{$2.2 \quad$ Associator identities}

There are various noteworthy identities for the associator that arise for the particular cochain twist induced by the constant $R$-flux background, which we summarise here as they will be used extensively in our calculations throughout this paper.

A main simplification is that the legs of the associator commute among themselves, $\phi_{a} \phi_{b}=\phi_{b} \phi_{a}$, and also with the legs of the twist, $\phi_{a} \mathrm{f}^{\alpha}=\mathrm{f}^{\alpha} \phi_{a}$ and $\phi_{a} \mathrm{f}_{\alpha}=\mathrm{f}_{\alpha} \phi_{a}$. Moreover, by antisymmetry of the trivector $R$, we have

$$
\phi_{a} \otimes \phi_{b} \otimes \phi_{c}=\bar{\phi}_{a} \otimes \bar{\phi}_{c} \otimes \bar{\phi}_{b}
$$

and $\phi_{a} \otimes \phi_{b} \phi_{c}=1 \otimes 1$, where here and in the following $(a, b, c)$ denotes a permutation of $(1,2,3)$. Furthermore, since the antipode is an antihomomorphism we have

$$
(\mathrm{id} \otimes \mathrm{id} \otimes S) \Phi=\exp \left(\frac{\ell_{s}^{3}}{6} R^{\mu \nu \rho} \partial_{\mu} \otimes \partial_{\nu} \otimes S\left(\partial_{\rho}\right)\right)=\exp \left(-\frac{\ell_{s}^{3}}{6} R^{\mu \nu \rho} \partial_{\mu} \otimes \partial_{\nu} \otimes \partial_{\rho}\right)
$$

which leads to

$$
\phi_{a} \otimes \phi_{b} \otimes S\left(\phi_{c}\right)=\bar{\phi}_{a} \otimes \bar{\phi}_{b} \otimes \bar{\phi}_{c}
$$

Since the coproduct $\Delta: U \operatorname{Vec}(\mathcal{M}) \rightarrow U \operatorname{Vec}(\mathcal{M}) \otimes U \operatorname{Vec}(\mathcal{M})$ is an algebra homomorphism, we have

$$
\begin{aligned}
(\mathrm{id} \otimes \mathrm{id} \otimes \Delta) \Phi & =\exp \left(\frac{\ell_{s}^{3}}{6} R^{\mu \nu \rho} \partial_{\mu} \otimes \partial_{\nu} \otimes \Delta\left(\partial_{\rho}\right)\right) \\
& =\exp \left(\frac{\ell_{s}^{3}}{6} R^{\mu \nu \rho}\left(\partial_{\mu} \otimes \partial_{\nu} \otimes \partial_{\rho} \otimes 1+\partial_{\mu} \otimes \partial_{\nu} \otimes 1 \otimes \partial_{\rho}\right)\right) \\
& =\exp \left(\frac{\ell_{s}^{3}}{6} R^{\mu \nu \rho} \partial_{\mu} \otimes \partial_{\nu} \otimes \partial_{\rho} \otimes 1\right) \exp \left(\frac{\ell_{s}^{3}}{6} R^{\mu \nu \rho} \partial_{\mu} \otimes \partial_{\nu} \otimes 1 \otimes \partial_{\rho}\right) \\
& =\phi_{1} \varphi_{1} \otimes \phi_{2} \varphi_{2} \otimes \phi_{3} \otimes \varphi_{3}
\end{aligned}
$$

where here and in the following we use different symbols for multiple associator insertions in order to avoid confusion. We further have (id $\otimes$ id $\otimes \Delta) \Phi=\left(\right.$ id $\otimes$ id $\left.\otimes \Delta_{\mathcal{F}}\right) \Phi$ because $\Delta_{\mathcal{F}}(\xi)=\mathcal{F} \Delta(\xi) \mathcal{F}^{-1}$ and $\mathcal{F}$ commutes with the legs of the associator. Hence we have

$$
\phi_{a} \otimes \phi_{b} \otimes \phi_{c_{(1)}} \otimes \phi_{c_{(2)}}=\phi_{a} \varphi_{a} \otimes \phi_{b} \varphi_{b} \otimes \phi_{c} \otimes \varphi_{c} .
$$

Finally, we also rewrite the identity $\Phi \Phi^{-1}=$ id as

$$
\phi_{a} \bar{\varphi}_{a} \otimes \phi_{b} \bar{\varphi}_{b} \otimes \phi_{c} \bar{\varphi}_{c}=1 \otimes 1 \otimes 1 .
$$




\subsection{Double field theory formulation}

Before deforming the geometry of $\mathcal{M}$ into a nonassociative differential geometry with the cochain twist $\mathcal{F}$ and the associated quasi-Hopf algebra $U \operatorname{Vec}^{\mathcal{F}}(\mathcal{M})$, let us describe the general extent to which our results will be applicable, particularly from the perspective of double field theory, as they will mostly be suppressed in the following in order to streamline our presentation and formulas.

Firstly, if globally non-geometric $Q$-flux is also present $[1,17,19,25]$, then it has the effect of modifying the twist element (2.3) to

$$
\mathcal{F}=F F_{R} F_{Q}
$$

where

$$
F_{Q}=\exp \left(-\frac{\mathrm{i} \kappa}{2} Q_{\rho \nu}^{\mu \nu}\left(w^{\rho} \partial_{\mu} \otimes \partial_{\nu}-\partial_{\nu} \otimes w^{\rho} \partial_{\mu}\right)\right)
$$

with $w^{\mu}$ closed string winding coordinates which may be regarded as momenta $\hat{p}^{\mu}$ conjugate to coordinates $\hat{x}_{\mu}$ that are T-dual to the spacetime variables $x^{\mu}$. The twist $F_{Q}$ is an abelian 2-cocycle, and the vector fields $w^{\rho} \partial_{\mu}$ commute with the other vector fields $\partial_{A}$ and $p_{\mu} \partial_{\nu}$ generating the twists $F$ and $F_{R}$, so the Hopf coboundary of (2.26) is still the associator (2.10); indeed, unlike the $R$-flux, the $Q$-flux only sources noncommutativity. In this setting the $Q$-flux and $R$-flux are independent deformation parameters, while coexistent constant $Q$-flux and $R$-flux in string theory are constrained in the absence of geometric fluxes by the Bianchi identities

$$
R^{\mu[\nu \rho} Q_{\mu}^{\lambda \tau]}=0 \quad \text { and } \quad Q_{\mu}^{[\nu \rho} Q_{\tau}^{\lambda] \mu}=0
$$

which are easily imposed as additional constraints on the twist element (2.26).

In fact, analogously to [8] one can extend the twist element (2.1) to the full phase space $\mathcal{M} \times \hat{\mathcal{M}}$ of double field theory in the $R$-flux frame as

$$
\hat{\mathcal{F}}=\mathcal{F} \hat{F},
$$

where

$$
\hat{F}=\exp \left(-\frac{\mathrm{i} \hbar}{2}\left(\hat{\partial}^{\mu} \otimes \tilde{\hat{\partial}}_{\mu}-\tilde{\hat{\partial}}_{\mu} \otimes \hat{\partial}^{\mu}\right)\right)
$$

implements the deformation of the canonical T-dual phase space $\hat{\mathcal{M}}$ with coordinates $\hat{x}_{\mu}, \hat{p}^{\mu}=w^{\mu}$. The cochain twist $(2.29)$ is $\mathrm{O}(2 d, 2 d)$-invariant so that it can be rotated to any other T-duality frame by using an $\mathrm{O}(2 d, 2 d)$ transformation on $\mathcal{M} \times \hat{\mathcal{M}}$, and in this way one can write down a nonassociative theory which is manifestly invariant under $\mathrm{O}(2 d, 2 d)$ rotations. In particular, by restricting to rotations which also preserve the canonical symplectic 2 -form of double phase space, we obtain a formulation that is invariant under a subgroup

$$
\mathrm{O}(d, d) \subseteq \mathrm{O}(2 d, 2 d) \cap \mathrm{Sp}(2 d)
$$


As the inclusion of $\hat{F}$ affects neither the commutation nor the association relations on the original phase space $\mathcal{M}$, while yielding the standard Moyal-Weyl deformation of the T-dual phase space $\hat{\mathcal{M}}$, we will regard the phase space $\hat{\mathcal{M}}$ as implicitly hidden in the background in all of our subsequent treatments, with the understanding that all of our formalism can be rotated to any T-duality frame by including a dependence on the T-dual coordinates of $\hat{\mathcal{M}}$ and suitably inserting $\hat{F}$ in formulas. In this way we obtain a manifestly $\mathrm{O}(d, d)$-invariant formulation of the gravity theory which follows.

From this perspective, there are also natural modifications of our formalism, analogous to those of Moyal-Weyl spaces [3], which fit nicely into the flux formulation of double field theory appropriate to curved backgrounds [17]. The vector fields

$$
X_{\mu}=\partial_{\mu}, \quad \tilde{X}^{\mu}=\tilde{\partial}^{\mu} \quad \text { and } \quad X_{\mu \nu}=p_{\mu} \partial_{\nu}-p_{\nu} \partial_{\mu}
$$

on $\mathcal{M}$, defining the twist deformation of flat space $M=\mathbb{R}^{d}$, represent a nilpotent subalgebra $\mathfrak{k}$ of the Lie algebra $\mathfrak{i s o}(2 d)$ with the nonvanishing Lie brackets

$$
\left[\tilde{X}^{\mu}, X_{\nu \rho}\right]=\delta^{\mu}{ }_{\nu} X_{\rho}-\delta_{\rho}^{\mu} X_{\nu} .
$$

For any collection of vector fields $\left\{X_{\mu}, \tilde{X}^{\mu}, X_{\mu \nu}, \mu, \nu=1, \ldots, d\right\}$ satisfying these Lie bracket relations on an arbitrary manifold $\mathcal{M}$, the cochain twist element

$$
\mathcal{F}_{\mathrm{c}}=\exp \left(-\frac{\mathrm{i} \hbar}{2}\left(X_{\mu} \otimes \tilde{X}^{\mu}-\tilde{X}^{\mu} \otimes X_{\mu}\right)-\frac{\mathrm{i} \kappa}{4} R^{\mu \nu \rho}\left(X_{\nu \rho} \otimes X_{\mu}-X_{\mu} \otimes X_{\nu \rho}\right)\right)
$$

of the Hopf algebra $U \mathfrak{i s o}(2 d) \subset U \operatorname{Vec}(\mathcal{M})$ provides a nonassociative deformation of $\mathcal{M}$, all of whose features fit into the framework we develop in the following.

For example, in the cases considered in the present paper we will see that there is a preferred basis $\partial_{A}, \mathrm{~d} x^{A}$ of vector fields and 1-forms on $\mathcal{M}$ that is invariant under the action of the associator and which greatly simplifies calculations. In particular, the Cartan structure equations expressing torsion and curvature as operators on vector fields will be established by checking that these operators define tensor fields, and by showing that in the preferred basis they coincide with the torsion and curvature coefficients. These simplifications can be carried out as well for the more general twist $\mathcal{F}_{\mathrm{c}}$, by considering as basis the commuting vector fields $X_{\mu}, \tilde{X}^{\mu}$ and their dual 1-forms; more generally, if the vector fields $X_{\mu}, \tilde{X}^{\mu}$ do not form a basis (e.g. they become degenerate), this can be achieved by completing them to a basis that is still invariant under the action of the associator with the methods described in ([3], section 4).

\section{Nonassociative deformation of tensor calculus}

\subsection{Principles of twist deformation}

The tensor algebra on $\mathcal{M}$ is covariant under the action of the universal enveloping algebra of infinitesimal diffeomorphisms $U \operatorname{Vec}(\mathcal{M})$. We have seen how the $R$-flux induces a twist deformation of the Hopf algebra $U \operatorname{Vec}(\mathcal{M})$ into the quasi-Hopf algebra $U \operatorname{Vec}^{\mathcal{F}}(\mathcal{M})$. We construct a nonassociative differential geometry on $\mathcal{M}$ by requiring it to be covariant with respect to the quasi-Hopf algebra $U \operatorname{Vec}^{\mathcal{F}}(\mathcal{M})$. 
Every time we have an algebra $\mathcal{A}$ that carries a representation of the Hopf algebra $U \operatorname{Vec}(\mathcal{M})$, and where vector fields $u \in \operatorname{Vec}(\mathcal{M})$ act on $\mathcal{A}$ as derivations: $u(a b)=$ $u(a) b+a u(b)$, i.e., every time we have a $U \operatorname{Vec}(\mathcal{M})$-module algebra $\mathcal{A}$, then deforming the multiplication in $\mathcal{A}$ into the star-multiplication

$$
a \star b=\overline{\mathrm{f}}^{\alpha}(a) \overline{\mathrm{f}}_{\alpha}(b)
$$

yields a noncommutative and nonassociative algebra $\mathcal{A}_{\star}$ that carries a representation of the quasi-Hopf algebra $U \mathrm{Vec}^{\mathcal{F}}(\mathcal{M})$, where

$$
\xi(a \star b)=\xi_{(1)}(a) \star \xi_{(2)}(b),
$$

for all $\xi \in U \operatorname{Vec}^{\mathcal{F}}(\mathcal{M})$ and $a, b \in \mathcal{A}$; in particular, the vector fields $\partial_{\mu}$ and $\tilde{\partial}^{\mu}$ act as deformed derivations according to the Leibniz rule implied by the coproduct (2.7). We say that $\mathcal{A}_{\star}$ is a $U \operatorname{Vec}^{\mathcal{F}}(\mathcal{M})$-module algebra because of the compatibility (3.2) of the action of $U \mathrm{Vec}^{\mathcal{F}}(\mathcal{M})$ with the product in $\mathcal{A}_{\star}$. For later use we recall the proof of the key property $(3.2)$ :

$$
\begin{aligned}
\xi(a \star b) & =\xi\left(\overline{\mathrm{f}}^{\alpha}(a) \overline{\mathrm{f}}_{\alpha}(b)\right) \\
& =\xi_{\left(1_{0}\right)}\left(\overline{\mathrm{f}}^{\alpha}(a)\right) \xi_{\left(2_{0}\right)}\left(\overline{\mathrm{f}}_{\alpha}(b)\right) \\
& =\overline{\mathrm{f}}^{\alpha}\left(\xi_{(1)}(a)\right) \overline{\mathrm{f}}_{\alpha}\left(\xi_{(2)}(b)\right) \\
& =\xi_{(1)}(a) \star \xi_{(2)}(b),
\end{aligned}
$$

where we used the notation $\Delta(\xi)=\xi_{\left(1_{0}\right)} \otimes \xi_{\left(2_{0}\right)}$ for the undeformed coproduct together with $\Delta(\xi) \mathcal{F}^{-1}=\mathcal{F}^{-1} \Delta_{\mathcal{F}}(\xi)$.

If the algebra $\mathcal{A}$ is commutative then the noncommutativity of $\mathcal{A}_{\star}$ is controlled by the $\mathcal{R}$-matrix as

$$
a \star b=\overline{\mathrm{R}}^{\alpha}(b) \star \overline{\mathrm{R}}_{\alpha}(a)=:{ }^{\alpha} b \star{ }_{\alpha} a,
$$

where in the last equality we used the notation ${ }^{\alpha} a:=\overline{\mathrm{R}}^{\alpha}(a)$ and ${ }_{\alpha} a:=\overline{\mathrm{R}}_{\alpha}(a)$; the expression (3.4) is easily proven by recalling that $\mathcal{R}=\mathcal{F}^{-2}$ and that $a \star b=\overline{\mathrm{f}}^{\alpha}(a) \overline{\mathrm{f}}_{\alpha}(b)=$ $\overline{\mathrm{f}}_{\alpha}(b) \overline{\mathrm{f}}^{\alpha}(a)$. If the algebra $\mathcal{A}$ is associative then the nonassociativity of $\mathcal{A}_{\star}$ is controlled by the associator $\Phi$ as

$$
(a \star b) \star c={ }^{\phi_{1}} a \star\left({ }^{\phi_{2}} b \star{ }^{\phi_{3}} c\right),
$$

where we denote ${ }^{\phi_{1}} a:=\phi_{1}(a)$; an explicit proof can be found in ([5], section 4.2).

In the following we deform the algebra of functions, the exterior algebra of differential forms and the algebra of tensor fields on $\mathcal{M}$ according to this prescription.

\section{$3.2 \quad$ Functions}

The action of a vector field $u \in \operatorname{Vec}(\mathcal{M})$ on a function $f \in C^{\infty}(\mathcal{M})$ is via the Lie derivative $\mathcal{L}_{u}(f)=u(f)$, which is indeed a derivation. The action of the Lie algebra $\operatorname{Vec}(\mathcal{M})$ on functions is extended to an action of the universal enveloping algebra $U \operatorname{Vec}(\mathcal{M})$ by defining the Lie derivative on products of vector fields as $\mathcal{L}_{u_{1} u_{2} \cdots u_{n}}:=\mathcal{L}_{u_{1}} \circ \mathcal{L}_{u_{2}} \circ \cdots \circ \mathcal{L}_{u_{n}}$ and 
by linearity. The $U \operatorname{Vec}(\mathcal{M})$-module algebra $C^{\infty}(\mathcal{M})$ (extended with power series in $\hbar$ and $\kappa)$ is then deformed to the $U \operatorname{Vec}^{\mathcal{F}}(\mathcal{M})$-module algebra $A_{\star}:=C^{\infty}(\mathcal{M})_{\star}$, which as a vector space is the same as $C^{\infty}(\mathcal{M})$ but with multiplication given by the star-product

$$
\begin{aligned}
f \star g & =\overline{\mathrm{f}}^{\alpha}(f) \cdot \overline{\mathrm{f}}_{\alpha}(g) \\
& =f \cdot g+\frac{\mathrm{i} \hbar}{2}\left(\partial_{\mu} f \cdot \tilde{\partial}^{\mu} g-\tilde{\partial}^{\mu} f \cdot \partial_{\mu} g\right)+\mathrm{i} \kappa R^{\mu \nu \rho} p_{\nu} \partial_{\rho} f \cdot \partial_{\mu} g+\cdots,
\end{aligned}
$$

where the ellipses denote terms of higher order in $\hbar$ and $\kappa$. Noncommutativity is controlled by the $\mathcal{R}$-matrix as $f \star g=\overline{\mathrm{R}}^{\alpha}(g) \star \overline{\mathrm{R}}_{\alpha}(f)={ }^{\alpha} g \star_{\alpha} f$, and nonassociativity by the associator $\Phi$ as $(f \star g) \star h={ }^{\phi_{1}} f \star\left({ }^{\phi_{2}} g \star{ }^{\phi_{3}} h\right)$. The constant function 1 on $\mathcal{M}$ is also the unit of $A_{\star}$ because $f \star 1=f=1 \star f$.

Denoting the star-commutator of functions by $[f, g]_{\star}:=f \star g-g \star f$, we reproduce in this way the defining phase space quasi-Poisson coordinate algebra

$$
\left[x^{\mu}, x^{\nu}\right]_{\star}=2 \mathrm{i} \kappa R^{\mu \nu \rho} p_{\rho}, \quad\left[x^{\mu}, p_{\nu}\right]_{\star}=\mathrm{i} \hbar \delta^{\mu}{ }_{\nu} \quad \text { and } \quad\left[p_{\mu}, p_{\nu}\right]_{\star}=0
$$

of the parabolic $R$-flux background, with the non-vanishing Jacobiators

$$
\left[x^{\mu}, x^{\nu}, x^{\rho}\right]_{\star}=\ell_{s}^{3} R^{\mu \nu \rho} .
$$

\subsection{Forms}

Similarly to $(3.6)$, we can deform the exterior algebra of differential forms $\Omega^{\sharp}(\mathcal{M})$ by introducing the star-exterior product

$$
\omega \wedge_{\star} \eta=\overline{\mathrm{f}}^{\alpha}(\omega) \wedge \overline{\mathrm{f}}_{\alpha}(\eta) .
$$

The algebra of differential forms with the nonassociative product $\Lambda_{\star}$ is denoted $\Omega_{\star}^{\sharp}$, with $\Omega_{\star}^{0}=A_{\star}$. Here too the vector fields in the twist act on differential forms via the Lie derivative; in particular, for the basis 1-forms we find

$$
\mathcal{L}_{\partial_{A}}\left(\mathrm{~d} x^{B}\right)=0
$$

along with (recalling that $\tilde{x}_{\mu}:=p_{\mu}$ )

$$
\mathcal{L}_{R^{\mu \nu \rho} X_{\nu \rho}}\left(\mathrm{d} x^{\sigma}\right)=2 R^{\mu \nu \sigma} \mathrm{d} \tilde{x}_{\nu} \quad \text { and } \quad \mathcal{L}_{R^{\mu \nu \rho} X_{\nu \rho}}\left(\mathrm{d} \tilde{x}_{\sigma}\right)=0,
$$

where the vector fields $X_{\mu \nu}$ are defined in (2.32) and we used the fact that the Lie derivative commutes with the exterior derivative. Iterating the commutativity of the exterior derivative $\mathrm{d}: \Omega_{\star}^{\sharp} \rightarrow \Omega_{\star}^{\sharp+1}$ with the Lie derivative along vector fields implies $\mathrm{d} \overline{\mathrm{f}}^{\alpha}(\omega)=\overline{\mathrm{f}}^{\alpha}(\mathrm{d} \omega)$ and $\mathrm{d} \overline{\mathrm{f}}_{\alpha}(\omega)=\overline{\mathrm{f}}_{\alpha}(\mathrm{d} \omega)$, giving the undeformed Leibniz rule

$$
\mathrm{d}\left(\omega \wedge_{\star} \eta\right)=\mathrm{d} \omega \wedge_{\star} \eta+(-1)^{|\omega|} \omega \wedge_{\star} \mathrm{d} \eta
$$

where $\omega$ is a homogeneous form of degree $|\omega|$.

The star-exterior product of 1 -forms $\mathrm{d} x^{A}$ reduces to the usual antisymmetric associative exterior product: using (3.10) we have

$$
\mathrm{d} x^{A} \wedge_{\star} \mathrm{d} x^{B}=\mathrm{d} x^{A} \wedge \mathrm{d} x^{B}=-\mathrm{d} x^{B} \wedge \mathrm{d} x^{A}=-\mathrm{d} x^{B} \wedge_{\star} \mathrm{d} x^{A} .
$$


In particular, the volume element is undeformed. For this, we note that the action of the associator (2.10) trivializes on the exterior products of basis 1-forms: in the case of three basis 1 -forms we obtain

$$
\begin{aligned}
\left(\mathrm{d} x^{A} \wedge_{\star} \mathrm{d} x^{B}\right) \wedge_{\star} \mathrm{d} x^{C} & ={ }^{\phi_{1}}\left(\mathrm{~d} x^{A}\right) \wedge_{\star}\left({ }^{\phi_{2}}\left(\mathrm{~d} x^{B}\right) \wedge_{\star}{ }^{\phi_{3}}\left(\mathrm{~d} x^{C}\right)\right) \\
& =\mathrm{d} x^{A} \wedge_{\star}\left(\mathrm{d} x^{B} \wedge_{\star} \mathrm{d} x^{C}\right) \\
& =\mathrm{d} x^{A} \wedge \mathrm{d} x^{B} \wedge \mathrm{d} x^{C}
\end{aligned}
$$

where $\phi_{a}$ act via Lie derivatives on forms and we used (3.10).

The exterior product between 0 -forms (functions) and 1-forms gives the space of 1forms the structure of a $C^{\infty}(\mathcal{M})$-bimodule. Similarly, restricting the star-exterior product of differential forms to functions and 1-forms defines the $A_{\star}$-bimodule structure of the space of 1 -forms $\Omega_{\star}^{1}$. In particular, the star-exterior product of functions and basis 1-forms is given by

$$
\begin{aligned}
& f \star \mathrm{d} \tilde{x}_{\mu}=f \cdot \mathrm{d} \tilde{x}_{\mu}=\mathrm{d} \tilde{x}_{\mu} \star f \\
& f \star \mathrm{d} x^{\mu}=f \cdot \mathrm{d} x^{\mu}-\frac{\mathrm{i} \kappa}{2} R^{\mu \nu \rho} \partial_{\nu} f \cdot \mathrm{d} \tilde{x}_{\rho}=\mathrm{d} x^{\mu} \star f-\mathrm{d} \tilde{x}_{\rho} \star \mathrm{i} \kappa R^{\mu \nu \rho} \partial_{\nu} f .
\end{aligned}
$$

Similarly to [14], it is convenient to package the relations in (3.15) into a single relation by defining an antisymmetric tensor $\mathscr{R}^{A B}{ }_{C}$ on $\mathcal{M}$ whose only non-vanishing components are $\mathscr{R}^{x^{\mu}, x^{\nu}}{ }_{\tilde{x}_{\rho}}=R^{\mu \nu \rho}$, so that

$$
f \star \mathrm{d} x^{A}=\mathrm{d} x^{C} \star\left(\delta^{A}{ }_{C} f-\mathrm{i} \kappa \mathscr{R}^{A B}{ }_{C} \partial_{B} f\right) .
$$

As a useful special case, this implies

$$
\mathrm{d} f=\partial_{A} f \mathrm{~d} x^{A}=\partial_{A} f \star \mathrm{d} x^{A}=\mathrm{d} x^{A} \star \partial_{A} f
$$

by antisymmetry of $R^{\mu \nu \rho}$ (and hence of $\mathscr{R}^{A B} C$ ).

\subsection{Tensors}

The usual tensor product $\otimes_{C^{\infty}(\mathcal{M})}$ over $C^{\infty}(\mathcal{M})$ is deformed to the star-tensor product $\otimes_{\star}$ over $A_{\star}$ defined by

$$
T \otimes_{\star} U=\overline{\mathrm{f}}^{\alpha}(T) \otimes_{C^{\infty}(\mathcal{M})} \overline{\mathrm{f}}_{\alpha}(U),
$$

where the action of the twist on the tensor fields $T$ and $U$ is via the Lie derivative. Due to nonassociativity, for $f \in A_{\star}$ one has

$$
(T \star f) \otimes_{\star} U={ }^{\phi_{1}} T \otimes_{\star}\left({ }^{\phi_{2}} f \star{ }^{\phi_{3}} U\right) .
$$

Here the star-tensor product $\otimes_{\star}$ between functions and tensor fields is denoted $\star$. In particular, it gives the space of vector fields $\operatorname{Vec}(\mathcal{M})$ an $A_{\star}$-bimodule structure. We denote $\operatorname{Vec}(\mathcal{M})$ with this $A_{\star}$-bimodule structure by $\operatorname{Vec}_{\star}$. In order to explicitly write the starproduct between functions and the basis vector fields $\partial_{\mu}, \tilde{\partial}^{\mu}$, we first compute the Lie 
derivative action of the vector fields in the twist on the basis vector fields (i.e., the Lie brackets):

$$
\mathcal{L}_{\partial_{A}}\left(\partial_{B}\right)=0
$$

together with

$$
\mathcal{L}_{R^{\mu \nu \rho} X_{\nu \rho}}\left(\partial_{\sigma}\right)=0 \quad \text { and } \quad \mathcal{L}_{R^{\mu \nu \rho} X_{\nu \rho}}\left(\tilde{\partial}^{\sigma}\right)=-2 R^{\mu \nu \sigma} \partial_{\nu}
$$

Then we have

$$
\begin{aligned}
& f \star \partial_{\mu}=f \cdot \partial_{\mu}=\partial_{\mu} \star f, \\
& f \star \tilde{\partial}^{\mu}=f \cdot \tilde{\partial}^{\mu}-\frac{\mathrm{i} \kappa}{2} R^{\mu \nu \rho} \partial_{\nu} f \cdot \partial_{\rho}=\tilde{\partial}^{\mu} \star f+\partial_{\nu} \star \mathrm{i} \kappa R^{\mu \nu \rho} \partial_{\rho} f,
\end{aligned}
$$

where here $\partial_{\mu} \star f$ denotes the right $A_{\star}$-action on $V_{e c}$ (and not the action of $\partial_{\mu}$ on the function $f$ ). Again we can write these relations collectively in the form

$$
f \star \partial_{A}=\partial_{C} \star\left(\delta^{C}{ }_{A} f+\mathrm{i} \kappa \mathscr{R}^{C B}{ }_{A} \partial_{B} f\right) .
$$

Using the star-tensor product, we can extend the $A_{\star}$-bimodule Vec ${ }_{\star}$ of vector fields to the $\Omega_{\star}^{\sharp}$-bimodule $V e c_{\star}^{\sharp}=\operatorname{Vec}_{\star} \otimes_{\star} \Omega_{\star}^{\sharp}$ : the left and right actions of the exterior algebra $\Omega_{\star}^{\sharp}$ on $\mathrm{Vec}_{\star}^{\sharp}$ are given by

$$
\begin{aligned}
& \left(u \otimes_{\star} \omega\right) \wedge_{\star} \eta={ }^{\phi_{1}} u \otimes_{\star}\left({ }^{\phi_{2}} \omega \wedge_{\star}{ }^{\phi_{3}} \eta\right), \\
& \eta \wedge_{\star}\left(u \otimes_{\star} \omega\right)={ }^{\alpha}\left(\bar{\phi}_{1}\left({ }_{\beta} u\right)\right) \otimes_{\star}\left(\alpha_{(1)}\left(\bar{\phi}_{2} \eta\right) \wedge_{\star}{ }^{\beta}\left(\alpha_{(2)}\left(\bar{\phi}_{3} \omega\right)\right)\right),
\end{aligned}
$$

where ${ }^{\alpha_{(a)}}(T):=\overline{\mathrm{R}}_{(a)}^{\alpha_{(a)}}(T)$ and $\alpha_{(a)} T:=\overline{\mathrm{R}}_{\alpha_{(a)}}(T)$ for $T$ a tensor or a form; the left action in (3.24) follows from

$$
\eta \wedge_{\star}\left(u \otimes_{\star} \omega\right)=\eta \wedge_{\star}\left({ }^{\beta} \omega \otimes_{\star} \beta\right)=\left(\bar{\phi}_{1} \eta \wedge_{\star} \bar{\phi}_{2} \beta \omega\right) \otimes_{\star} \bar{\phi}_{3}{ }_{\beta} u={ }^{\alpha}\left(\bar{\phi}_{3}{ }_{\beta} u\right) \otimes_{\star}\left({ }^{\bar{\phi}_{1}} \eta \wedge_{\star} \bar{\phi}_{2} \beta \omega\right)
$$

where in the first equality we used the fact that the tensor product between contravariant and covariant tensors is commutative, in particular $u \otimes_{C^{\infty}(\mathcal{M})} \omega=\omega \otimes_{C^{\infty}(\mathcal{M})} u$, and similarly in the last equality.

Analogously, we can extend the $A_{\star}$-bimodule of 1-forms $\Omega_{\star}^{1}$ to an $\Omega_{\star}^{\sharp}$-bimodule $\Omega_{\star}^{\sharp} \otimes_{\star} \Omega_{\star}^{1}$.

\subsection{Duality}

The three star-multiplications $\star, \Lambda_{\star}$ and $\otimes_{\star}$ thus far constructed are compatible with the $U \operatorname{Vec}^{\mathcal{F}}(\mathcal{M})$-action according to (3.2). This compatibility can be regarded as equivariance of these products under the $U \mathrm{Vec}^{\mathcal{F}}(\mathcal{M})$-action: there is no action of $\xi$ on the star-multiplication in (3.2), only on (the functions, forms or tensors) $a$ and $b$. This notion of equivariance under the universal enveloping algebra of diffeomorphisms $U \operatorname{Vec}^{\mathcal{F}}(\mathcal{M})$ (invariance and covariance in physics parlance) is the guiding principle in constructing a noncommutative and nonassociative differential geometry on $\mathcal{M}$. The recipe thus far considered, which consists in deforming a multiplication $m$ to the star-multiplication $\star$ defined 
by composing the classical product with the inverse twist, $\star:=m \circ \mathcal{F}^{-1}$, extends more generally to any bilinear map that is equivariant under infinitesimal diffeomorphisms, i.e., under $U \operatorname{Vec}(\mathcal{M})$.

For example, the pairing between 1-forms and vectors $\langle, \quad\rangle: \Omega^{1}(\mathcal{M}) \times \operatorname{Vec}(\mathcal{M}) \rightarrow$ $C^{\infty}(\mathcal{M})$ is deformed to the star-pairing

$$
\langle,\rangle_{\star}:=\langle,\rangle \circ \mathcal{F}^{-1}: \Omega_{\star}^{1} \times \operatorname{Vec}_{\star} \longrightarrow C^{\infty}(\mathcal{M}),
$$

which is explicitly given by

$$
\langle\omega, u\rangle_{\star}=\left\langle\overline{\mathrm{f}}^{\alpha}(\omega), \overline{\mathrm{f}}_{\alpha}(u)\right\rangle .
$$

Equivariance of the star-pairing under the quasi-Hopf algebra $U \operatorname{Vec}^{\mathcal{F}}(\mathcal{M})$,

$$
\xi\langle\omega, u\rangle_{\star}=\left\langle\xi_{(1)} \omega, \xi_{(2)} u\right\rangle_{\star},
$$

follows from equivariance of the undeformed pairing under the $\operatorname{Hopf}$ algebra $U \operatorname{Vec}(\mathcal{M})$, with the proof being analogous to (3.3).

Since the usual pairing is $C^{\infty}(\mathcal{M})$-linear: $\langle\omega \cdot f, u\rangle=\langle\omega, f \cdot u\rangle,\langle f \cdot \omega, u\rangle=$ $f \cdot\langle\omega, u\rangle$, and $\langle\omega, u \cdot f\rangle=\langle\omega, u\rangle \cdot f$, it follows that the star-pairing is $A_{\star}$-linear:

$$
\begin{aligned}
& \langle\omega \star f, u\rangle_{\star}=\left\langle{ }^{\phi_{1}} \omega,{ }^{\phi_{2}} f \star{ }^{\phi_{3}} u\right\rangle_{\star}, \\
& \langle f \star \omega, u\rangle_{\star}={ }^{\phi_{1}} f \star\left\langle{ }^{\phi_{2}} \omega,{ }^{\phi_{3}} u\right\rangle_{\star}, \\
& \langle\omega, u \star f\rangle_{\star}=\left\langle{ }^{\bar{\phi}_{1}} \omega, \bar{\phi}^{\bar{\phi}_{2}} u\right\rangle_{\star}{ }^{\bar{\phi}_{3}} f,
\end{aligned}
$$

with the proof being analogous to that of quasi-associativity (3.19) of the star-tensor product. The first equality in (3.29) shows that the star-pairing is a well-defined map

$$
\langle\rangle_{\star}: \Omega_{\star}^{1} \otimes_{\star} \operatorname{Vec}_{\star} \longrightarrow A_{\star} .
$$

At zeroth order in the deformation parameters $\hbar$ and $\kappa$, this is just the canonical undeformed pairing \langle\rangle of 1 -forms with vector fields which is nondegenerate, and hence the star-pairing \langle\rangle$_{\star}$ is nondegenerate as well. Because of (3.10) and (3.20), the star-pairing between basis vector fields $\partial_{A}$ and basis 1-forms $\mathrm{d} x^{A}$ is undeformed: $\left\langle\mathrm{d} x^{A}, \partial_{B}\right\rangle_{\star}=\delta^{A}{ }_{B}$.

This pairing can be extended to star-tensor products

$$
\langle\rangle_{\star}:\left(\Omega_{\star}^{1} \otimes_{\star} \Omega_{\star}^{1}\right) \otimes_{\star}\left(\operatorname{Vec}_{\star} \otimes_{\star} \operatorname{Vec}_{\star}\right) \longrightarrow A_{\star}
$$

in the following way. Firstly, for $\omega, \eta \in \Omega_{\star}^{1}$ and $u \in \mathrm{Vec}_{\star}$ we define the 1-form

$$
\left\langle\left(\omega \otimes_{\star} \eta\right), u\right\rangle_{\star}:={ }^{\phi_{1}} \omega \star\left\langle{ }^{\phi_{2}} \eta,{ }^{\phi_{3}} u\right\rangle_{\star} .
$$

This definition is compatible with equivariance under the quasi-Hopf algebra action, since for $\xi \in U \operatorname{Vec}^{\mathcal{F}}(\mathcal{M})$ we have

$$
\begin{aligned}
\left.\xi_{\langle}\left(\omega \otimes_{\star} \eta\right), u\right\rangle_{\star} & \left.=\xi^{\phi_{1}} \omega \star\left\langle{ }^{\phi_{2}} \eta,{ }^{\phi_{3}} u\right\rangle_{\star}\right) \\
& =\xi_{(1)} \phi_{1} \omega \star \xi_{(2)}\left\langle{ }^{\phi_{2}} \eta,{ }^{\phi_{3}} u\right\rangle_{\star} \\
& =\xi_{(1)} \phi_{1} \omega \star\left\langle{ }_{(2)(1)}{ }^{\phi_{2}} \eta,{ }^{\xi_{(2)}(2)}{ }^{\phi_{3}} u\right\rangle_{\star} \\
& ={ }^{\phi_{1} \xi_{(1)}(1) \omega \star\left\langle{ }^{\phi_{2}} \xi_{(1)}(2)\right.} \eta,{ }^{\left.\phi_{3} \xi_{(2)} u\right\rangle_{\star}} \\
& =\left\langle\xi_{(1)}\left(\omega \otimes_{\star} \eta\right),{ }^{\xi_{(2)}} u\right\rangle_{\star}
\end{aligned}
$$


where in the second line we used the equivariance of the star-product, in the third line the equivariance of the star-pairing, and in the fourth line the quasi-coassociativity property (2.12). We then define the pairing

$$
\begin{aligned}
& \left\langle\left(\omega \otimes_{\star} \eta\right),\left(u \otimes_{\star} v\right)\right\rangle_{\star}:=\left\langle\left\langle\bar{\phi}_{1}\left(\omega \otimes_{\star} \eta\right),{ }^{\bar{\phi}_{2}} u\right\rangle_{\star},{ }^{\bar{\phi}_{3}} v\right\rangle_{\star} \\
& =\left\langle\left\langle\bar{\phi}_{1} \omega \otimes_{\star} \bar{\varphi}_{1} \eta, \bar{\phi}_{2} \bar{\varphi}_{2} u\right\rangle_{\star}, \bar{\phi}_{3} \bar{\varphi}_{3} v\right\rangle_{\star} \\
& =\left\langle\zeta_{1} \bar{\phi}_{1} \omega \star\left\langle\zeta_{2} \bar{\varphi}_{1} \eta, \zeta_{3} \bar{\phi}_{2} u\right\rangle_{\star}, \bar{\phi}_{3} \bar{\varphi}_{3} v\right\rangle_{\star}
\end{aligned}
$$

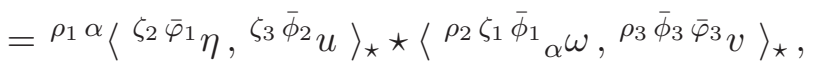

and one again checks that it is equivariant under $U \operatorname{Vec}^{\mathcal{F}}(\mathcal{M})$ by using quasicoassociativity (2.12). This definition can be straightforwardly iterated to arbitrary startensor products.

\subsection{Module homomorphisms}

Tensors can be regarded either as sections of vector bundles or as maps between sections of vector bundles. In section 3.4 we have taken the first point of view and deformed the product of sections to the star-tensor product. Thanks to the pairing $\langle,\rangle_{\star}$, we can also consider the second perspective; for example, for any 1-form $\omega$ the object $\langle\omega,\rangle_{\star}$ is a right $A_{\star}$-linear map from the $A_{\star}$-bimodule $V_{e c}$ to $A_{\star}$. More generally, given $A_{\star}$-bimodules $V_{\star}$ and $W_{\star}$, we can consider the space of module homomorphisms (linear maps) hom $\left(V_{\star}, W_{\star}\right)$. This space carries the adjoint action of the Hopf algebra, which is given by

$$
{ }^{\xi} L(v):=\left({ }^{\xi} L\right)(v)=\xi_{(1)}\left(L\left(S\left(\xi_{(2)}\right)(v)\right)\right),
$$

for $\xi \in U \operatorname{Vec}^{\mathcal{F}}(\mathcal{M}), L \in \operatorname{hom}\left(V_{\star}, W_{\star}\right)$ and $v \in V_{\star}$. It is straightforward to check equivariance of the evaluation of $L$ on $v$ :

$$
\xi(L(v))=\xi_{(1)} L\left(\xi_{(2)} v\right) .
$$

Indeed the right-hand side can be written as

$$
\begin{aligned}
& \xi_{(1)} L\left(\xi_{(2)} v\right)={ }^{\xi_{(1)}(1)}\left(L\left(^{S\left(\xi_{(1)}\right)} \xi_{(2)} v\right)\right) \\
& =\bar{\phi}_{1} \xi_{(1)} \varphi_{1}\left(L\left({ }^{\left.S\left(\bar{\phi}_{2} \xi_{(2)}\right)_{(1)} \varphi_{2}\right) \bar{\phi}_{3} \xi_{(2)}{ }_{(2)} \varphi_{3}} v\right)\right) \\
& =\xi_{1} \varphi_{1}\left(L\left(^{\left.\left.\varphi_{2} S\left(\xi_{(2)}\right)_{(1)}\right) \xi_{(2)}\right)_{(2)} \varphi_{3}} v\right)\right) \\
& =\xi(L(v)),
\end{aligned}
$$

where we used (2.12), antimultiplicativity of the antipode $S$, the compatibility (2.15) and $\phi_{a} \otimes \phi_{b} \phi_{c}=1 \otimes 1$. Since the vector fields comprising the associator commute with those of the twisting cochain $\mathcal{F}$, using $(2.22)$ and $\phi_{a} \otimes \phi_{b} \phi_{c}=1 \otimes 1$ we obtain the following identities that will be frequently used:

$$
\begin{aligned}
& \phi_{a} \otimes \phi^{\phi_{b}}\left(v \star^{\phi_{c}} f\right)=\phi_{a} \otimes\left({ }^{\phi_{b}} v \star^{\phi_{c}} f\right), \\
& { }^{\phi_{a}} L\left({ }^{\phi_{b}} v\right) \otimes \phi_{c}={ }^{\phi_{a}}\left(L\left({ }^{\phi_{b}} v\right)\right) \otimes \phi_{c},
\end{aligned}
$$

for $v \in V_{\star}, f \in A_{\star}$ and $L \in \operatorname{hom}\left(V_{\star}, W_{\star}\right)$. 
We define the composition of homomorphisms by

$$
\left(L_{1} \bullet L_{2}\right)(v):={ }^{\phi_{1}} L_{1}\left({ }^{\phi_{2}} L_{2}\left({ }^{\phi_{3}} v\right)\right)
$$

for $L_{1} \in \operatorname{hom}\left(W_{\star}, X_{\star}\right), L_{2} \in \operatorname{hom}\left(V_{\star}, W_{\star}\right)$ and $v \in V_{\star}$. One can readily check equivariance of this composition, i.e., compatibility with the $U \operatorname{Vec}^{\mathcal{F}}(\mathcal{M})$-action:

$$
\xi\left(L_{1} \bullet L_{2}\right)=\xi_{(1)} L_{1} \bullet \xi_{(2)} L_{2},
$$

with the proof being similar to (3.37), see also [10]. In particular, with this composition the $U \operatorname{Vec}^{\mathcal{F}}(\mathcal{M})$-module end $\left(V_{\star}\right)$ of linear maps on $V_{\star}$ is a quasi-associative algebra:

$$
\left(L_{1} \bullet L_{2}\right) \bullet L_{3}={ }^{\phi_{1}} L_{1} \bullet\left({ }^{\phi_{2}} L_{2} \bullet{ }^{\phi_{3}} L_{3}\right),
$$

for all $L_{1}, L_{2}, L_{3} \in \operatorname{end}\left(V_{\star}\right)$. We define the twisted commutator of endomorphisms $L_{1}, L_{2} \in$ $\operatorname{end}\left(V_{\star}\right)$ through

$$
\left[L_{1}, L_{2}\right] \bullet=L_{1} \bullet L_{2}-{ }^{\alpha} L_{2} \bullet{ }_{\alpha} L_{1},
$$

where the braiding with the $\mathcal{R}$-matrix ensures equivariance of $[,] \bullet$ under the $U \operatorname{Vec}^{\mathcal{F}}(\mathcal{M})$ action: ${ }^{\xi}\left[L_{1}, L_{2}\right] \bullet=\left[\xi_{(1)} L_{1},{ }_{(2)} L_{2}\right] \bullet$

A map $L \in \operatorname{hom}\left(V_{\star}, W_{\star}\right)$ is right $A_{\star}$-linear if

$$
L(v \star f)={ }^{\bar{\phi}_{1}} L\left(\bar{\phi}_{2} v\right) \star{ }^{\bar{\phi}_{3}} f=\bar{\phi}_{1}\left(L\left(\bar{\phi}_{2} v\right)\right) \star{ }^{\bar{\phi}_{3}} f .
$$

We denote the space of all such maps by $\operatorname{hom}_{\star}\left(V_{\star}, W_{\star}\right)$; it closes under the $U \operatorname{Vec}^{\mathcal{F}}(\mathcal{M})$ action [10]. To see this explicitly, we need to show that if $L$ is right $A_{\star}$-linear, then so is ${ }^{\xi} L$ for all $\xi \in U \operatorname{Vec}^{\mathcal{F}}(\mathcal{M})$. This follows from the calculation

$$
\begin{aligned}
& { }^{\xi} L(v \star f)=\xi_{(1)}\left(L\left({ }^{S\left(\xi_{(2)}(2)\right.} v \star^{S\left(\xi_{(2)}(1)\right.} f\right)\right) \\
& \left.=\xi_{(1)} \bar{\phi}_{1}\left[\left(L\left(^{\bar{\phi}_{2} S\left(\xi_{(2)(2)}\right)} v\right)\right) \star \bar{\phi}_{3} S\left(\xi_{(2)}\right)_{(1)}\right) f\right] \\
& ={ }^{\phi_{1} \xi_{(1)}(1)}\left[\left(L\left(S\left(\phi_{3} \xi_{(2)}\right) v\right)\right) \star S\left(\phi_{2} \xi_{(1)}{ }_{(2)}\right) f\right]
\end{aligned}
$$

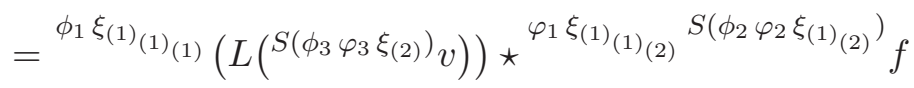

$$
\begin{aligned}
& ={ }^{\left.\phi_{1} \bar{\eta}_{1} \xi_{(1)}\right)_{(1)} \rho_{1}}\left(L\left(S\left(\phi_{3} \varphi_{3} \xi_{(2)}\right) v\right)\right) \star{ }^{\varphi_{1} \bar{\eta}_{2} \xi_{(1)}(2)(1)} \rho_{2} S\left(\phi_{2} \varphi_{2} \bar{\eta}_{3} \xi_{(1)(2)(2)}{ }^{\left.\rho_{3}\right)} f\right. \\
& ={ }^{\phi_{1} \bar{\eta}_{1} \xi_{(1)}(1)}\left(L\left(S\left(\phi_{3} \varphi_{3} \xi_{(2)}\right) v\right)\right) \star^{\left.\varphi_{1} \bar{\eta}_{2} \xi_{(1)(2)(1)} S\left(\xi_{(1)}\right)_{(2)}\right) S\left(\bar{\eta}_{3} \varphi_{2} \phi_{2}\right)} f \\
& =\phi_{1} \xi_{(1)}\left(L\left(S\left(\xi_{(2)}\right) S\left(\phi_{3}\right) u\right)\right) \star^{S\left(\phi_{2}\right)} f \\
& =\bar{\phi}_{1}(\xi)\left({ }^{\xi}{ }^{2} v\right) \star{ }^{\bar{\phi}_{3}} f \text {, }
\end{aligned}
$$

where the third equality follows from (2.12), antimultiplicativity of the antipode $S$, and (2.20).

For later use, let us explicitly demonstrate that the composition of $L_{1} \in \operatorname{hom}_{\star}\left(W_{\star}, X_{\star}\right)$ and $L_{2} \in \operatorname{hom}_{\star}\left(V_{\star}, W_{\star}\right)$ is a right $A_{\star}$-linear map $L_{1} \bullet L_{2} \in \operatorname{hom}_{\star}\left(V_{\star}, X_{\star}\right)$; see [10] for a general proof in the setting of arbitrary quasi-Hopf algebras. For this, we compute

$$
\left(L_{1} \bullet L_{2}\right)(v \star f)={ }^{\phi_{1}}\left(L_{1}\left({ }^{\phi_{2}}\left(L_{2}\left({ }^{\phi_{3}}(v \star f)\right)\right)\right)\right)
$$


using ${ }^{\phi_{3}}(v \star f)={ }^{\phi_{3(1)}} v \star^{\phi_{3}(2)} f$ and the identity (2.24) to get

$$
\begin{aligned}
\left(L_{1} \bullet L_{2}\right)(v \star f) & ={ }^{\phi_{1} \varphi_{1}}\left(L_{1}\left(\phi_{2} \varphi_{2}\left(L_{2}\left({ }^{\phi_{3}} v \star{ }^{\varphi_{3}} f\right)\right)\right)\right) \\
& =\phi_{1} \varphi_{1}\left(L_{1}\left(\phi_{2} \varphi_{2}\left[\bar{\rho}_{1}\left(L_{2}\left(\bar{\rho}_{2} \phi_{3} v\right)\right) \star \bar{\rho}_{3} \varphi_{3} f\right]\right)\right) \\
& =\phi_{1} \varphi_{1}\left(L_{1}\left({ }^{\phi_{2}(1)}\left[{ }^{\varphi_{2}} \bar{\rho}_{1}\left(L_{2}\left(\bar{\rho}_{2} \phi_{3} v\right)\right)\right] \star{ }^{\phi_{2}(2)} \bar{\rho}_{3} \varphi_{3} f\right)\right) \\
& =\phi_{1} \check{\phi}_{1} \varphi_{1}\left(L_{1}\left(\phi_{2} \varphi_{2} \bar{\rho}_{1}\left(L_{2}\left(\bar{\rho}_{2} \phi_{3} \check{\phi}_{3} v\right)\right) \star \check{\phi}_{2} \bar{\rho}_{3} \varphi_{3} f\right)\right) \\
& =\phi_{1} \check{\phi}_{1} \varphi_{1} \bar{\zeta}_{1}\left(L_{1}\left(\bar{\zeta}_{2}\left[\phi_{2} \varphi_{2} \bar{\rho}_{1}\left(L_{2}\left(\bar{\rho}_{2} \phi_{3} \check{\phi}_{3} v\right)\right)\right]\right)\right) \star \bar{\zeta}_{3} \check{\phi}_{2} \bar{\rho}_{3} \varphi_{3} f \\
& =\phi_{1} \check{\phi}_{1} \varphi_{1} \bar{\zeta}_{1}\left(L_{1}\left(\bar{\zeta}_{2}\left[\phi_{2} \check{\varphi}_{2} \varphi_{2} \bar{\rho}_{1}\left(L_{2}\left(\bar{\rho}_{2} \phi_{3} \check{\phi}_{3} \check{\varphi}_{3} v\right)\right)\right]\right)\right) \star \check{\varphi}_{1} \bar{\zeta}_{3} \check{\phi}_{2} \bar{\rho}_{3} \varphi_{3} f \\
& ={ } \check{\phi}_{1}\left(L_{1}\left(\phi_{2}\left(L_{2}\left({ }^{\phi_{3} \check{\phi}_{3}} v\right)\right)\right)\right) \star \check{\phi}_{2} f \\
& =\phi_{1} \bar{\phi}_{1}\left(L_{1}\left(\phi_{2}\left(L_{2}\left({ }^{\phi_{3}} \bar{\phi}_{2} v\right)\right)\right)\right) \star \bar{\phi}_{3} f \\
& =\bar{\phi}_{1}\left(\left(L_{1} \bullet L_{2}\right)\left(\bar{\phi}_{2} v\right)\right) \star \bar{\phi}_{3} f,
\end{aligned}
$$

which establishes that $L_{1} \bullet L_{2}$ is right $A_{\star}$-linear.

For later use in our constructions of connections and curvature, we will also prove some properties of tensor products of right $A_{\star}$-linear maps. Let $U_{\star}, V_{\star}$ and $W_{\star}$ be $A_{\star}$-bimodules. Then the lifting of $L \in \operatorname{hom}_{\star}\left(U_{\star}, W_{\star}\right)$ to $L \otimes$ id $\in \operatorname{hom}_{\star}\left(U_{\star} \otimes_{\star} V_{\star}, W_{\star} \otimes_{\star} V_{\star}\right)$ is defined by

$$
(L \otimes \mathrm{id})\left(u \otimes_{\star} v\right):=\left(\bar{\phi}_{1} L\right)\left(\bar{\phi}_{2} u\right) \otimes_{\star} \bar{\phi}_{3} v=\bar{\phi}_{1}\left(L\left(\bar{\phi}_{2} u\right)\right) \otimes_{\star} \bar{\phi}_{3} v
$$

for $u \in U_{\star}$ and $v \in V_{\star}$. Let us first check equivariance:

$$
\xi(L \otimes \mathrm{id})={ }^{\xi} L \otimes \mathrm{id} .
$$

For this, we need to check that

$$
\xi\left((L \otimes \mathrm{id})\left(u \otimes_{\star} v\right)\right)=\xi_{(1)}(L \otimes \mathrm{id})\left(\xi_{(2)}\left(u \otimes_{\star} v\right)\right)=\left(\xi_{(1)} L \otimes \mathrm{id}\right)\left(\xi_{(2)}\left(u \otimes_{\star} v\right)\right)
$$

for arbitrary $u, v$ and for any $\xi \in U \operatorname{Vec}^{\mathcal{F}}(\mathcal{M})$. This follows from the calculation

$$
\begin{aligned}
& \xi\left((L \otimes \mathrm{id})\left(u \otimes_{\star} v\right)\right)=\xi_{(1)}\left(\bar{\phi}_{1} L\left(\bar{\phi}_{2} u\right)\right) \otimes_{\star} \xi_{(2)} \bar{\phi}_{3} v \\
& =\left({ }^{\xi_{(1)}{ }_{(1)} \bar{\phi}_{1}} L\right)\left({ }^{\xi_{(1)}(2)} \bar{\phi}_{2} u\right) \otimes_{\star} \xi_{(2)} \bar{\phi}_{3} v \\
& =\left({ }^{\bar{\varphi}_{1} \varphi_{1} \xi_{(1)}{ }_{(1)} \bar{\phi}_{1}} L\right)\left({ }^{\bar{\varphi}_{2} \varphi_{2} \xi_{(1)}(2)} \bar{\phi}_{2} u\right) \otimes_{\star} \bar{\varphi}_{3} \varphi_{3} \xi_{(2)} \bar{\phi}_{3} v \\
& =\left({ }^{\varphi_{1} \xi_{(1)(1)} \bar{\phi}_{1}} L \otimes \mathrm{id}\right)\left({ }^{\varphi_{2} \xi_{(1)} \bar{\phi}_{(2)}} u \otimes_{\star} \varphi_{3} \xi_{(2)} \bar{\phi}_{3} v\right) \\
& =\left(\varphi_{1} \bar{\phi}_{1} \xi_{(1)} L \otimes \mathrm{id}\right)\left(^{\varphi_{2} \bar{\phi}_{2} \xi_{(2)}(1)} u \otimes_{\star}{ }^{\varphi_{3} \bar{\phi}_{3} \xi_{(2)}(2)} v\right) \\
& =\left(\xi_{(1)} L \otimes \mathrm{id}\right)\left(\xi_{(2)}\left(u \otimes_{\star} v\right)\right) \text {. }
\end{aligned}
$$

With the definition (3.48) the map $L \otimes$ id is indeed well-defined on $U_{\star} \otimes_{\star} V_{\star}$ :

$$
(L \otimes \mathrm{id})\left((u \star f) \otimes_{\star} v\right)=(L \otimes \mathrm{id})\left({ }^{\phi_{1}} u \otimes_{\star}\left({ }^{\phi_{2}} f \star{ }^{\phi_{3}} v\right)\right) .
$$


For this, we use right $A_{\star}$-linearity of $L$ to write the left-hand side as

$$
(L \otimes \mathrm{id})\left((u \star f) \otimes_{\star} v\right)={ }^{\bar{\phi}_{1} \bar{\varphi}_{1}} L\left({ }^{\tau_{1}} \bar{\phi}_{2} \bar{\varphi}_{2} u\right) \otimes_{\star}\left(\bar{\varphi}_{3} \tau_{2} f \star{ }^{\tau_{3} \bar{\phi}_{3}} v\right)
$$

which is indeed equal to the right-hand side

$$
(L \otimes \mathrm{id})\left({ }^{\phi_{1}} u \otimes_{\star}\left({ }^{\phi_{2}} f \star{ }^{\phi_{3}} v\right)\right)={ }^{\bar{\varphi}_{1}} L\left(\bar{\varphi}_{2} \phi_{1} u\right) \otimes_{\star} \bar{\varphi}_{3}\left(\phi_{2} f \star{ }^{\phi_{3}} v\right) .
$$

Finally, we can show that $L \otimes$ id is right $A_{\star}$-linear:

$$
(L \otimes \mathrm{id})\left(\left(u \otimes_{\star} v\right) \star f\right)=\left(\bar{\zeta}_{1}(L \otimes \mathrm{id})^{\bar{\zeta}_{2}}(u \otimes v)\right) \star \bar{\zeta}_{3} f .
$$

For this, we note that the left-hand side can be expressed as

$$
(L \otimes \mathrm{id})\left(\left(u \otimes_{\star} v\right) \star f\right)={ }^{\bar{\phi}_{1} \bar{\varphi}_{1}} L\left(\bar{\phi}_{2} \bar{\varphi}_{2} \zeta_{1} u\right) \otimes_{\star}\left(\bar{\phi}_{3} \zeta_{2} v \star{ }^{\bar{\varphi}_{3} \zeta_{3}} f\right)
$$

which is indeed equal to the right-hand side

$$
\left(\bar{\zeta}_{1}(L \otimes \mathrm{id})^{\bar{\zeta}_{2}}\left(u \otimes_{\star} v\right)\right) \star{ }^{\bar{\zeta}_{3}} f=\varphi_{1} \bar{\phi}_{1} \bar{\zeta}_{1} \bar{\rho}_{1} L\left({ }^{\tau_{1}} \bar{\phi}_{2} \bar{\zeta}_{2} u\right) \otimes_{\star}\left(\varphi_{2} \tau_{2} \bar{\phi}_{3} \bar{\rho}_{2} v \star \varphi_{3} \tau_{3} \bar{\rho}_{3} \bar{\zeta}_{3} f\right) .
$$

We also define

$$
\text { id } \otimes_{\mathcal{R}} L:=\tau_{\mathcal{R}} \bullet(L \otimes \mathrm{id}) \bullet \tau_{\mathcal{R}},
$$

with $\tau_{\mathcal{R}}\left(v \otimes_{\star} u\right)={ }^{\alpha} u \otimes_{\star \alpha} v$ the braiding operator. This definition is well-posed because $\tau_{\mathcal{R}}$ is compatible with (3.19), and the twisted composition $\bullet$ is associative if one of the maps is equivariant (as $\left.\phi_{1} \otimes \phi_{2} \phi_{3}=1 \otimes 1\right)$. Moreover, $\tau_{\mathcal{R}}$ is an equivariant map: ${ }^{\xi}\left(\tau_{\mathcal{R}}\left(u \otimes_{\star} v\right)\right)=$ $\tau_{\mathcal{R}}\left({ }^{\xi}\left(u \otimes_{\star} v\right)\right)$, and thus the lifting of $L$ to id $\otimes_{\mathcal{R}} L$ is equivariant: ${ }^{\xi}\left(\operatorname{id} \otimes_{\mathcal{R}} L\right)=\tau_{\mathcal{R}} \bullet\left({ }^{\xi} L \otimes\right.$ id) $\bullet \tau_{\mathcal{R}}=$ id $\otimes_{\mathcal{R}} \xi L$. The lift id $\otimes_{\mathcal{R}} L$ is furthermore right $A_{\star}$-linear:

$$
\begin{aligned}
\left(\operatorname{id} \otimes_{\mathcal{R}} L\right)\left(\left(u \otimes_{\star} v\right) \star f\right) & =\tau_{\mathcal{R}}\left(\left(\bar{\phi}_{1}(L \otimes \mathrm{id}) \tau_{\mathcal{R}} \bar{\phi}_{2}\left(u \otimes_{\star} v\right)\right) \star{ }^{\bar{\phi}_{3}} f\right) \\
& =\left(\tau_{\mathcal{R}}\left(\bar{\phi}_{1} L \otimes \mathrm{id}\right) \tau_{\mathcal{R}} \bar{\phi}_{2}\left(u \otimes_{\star} v\right)\right) \star{ }^{\bar{\phi}_{3}} f \\
& =\left({ }^{\bar{\phi}_{1}}\left(\mathrm{id} \otimes_{\mathcal{R}} L\right)^{\bar{\phi}_{2}}\left(u \otimes_{\star} v\right)\right) \star{ }^{\bar{\phi}_{3}} f,
\end{aligned}
$$

where we used right $A_{\star}$-linearity of $L \otimes \mathrm{id}$.

To summarise, if $L: U_{\star} \rightarrow W_{\star}$ is right $A_{\star}$-linear, then $L \otimes$ id is well-defined on $U_{\star} \otimes_{\star} V_{\star}$ and right $A_{\star}$-linear, and hence so is id $\otimes_{\mathcal{R}} L$. In particular, given another right $A_{\star}$-linear map $L^{\prime}: V_{\star}^{\prime} \rightarrow W_{\star}^{\prime}$ we obtain a well-defined right $A_{\star}$-linear map $L \otimes_{\mathcal{R}} L^{\prime}:=(L \otimes \mathrm{id}) \bullet\left(\operatorname{id} \otimes_{\mathcal{R}} L^{\prime}\right)$, which is compatible with the action of $U \operatorname{Vec}^{\mathcal{F}}(\mathcal{M})$ and is quasi-associative [10]:

$$
\left(L \otimes_{\mathcal{R}} L^{\prime}\right) \otimes_{\mathcal{R}} L^{\prime \prime}=\Phi^{-1} \bullet\left(L \otimes_{\mathcal{R}}\left(L^{\prime} \otimes_{\mathcal{R}} L^{\prime \prime}\right)\right) \bullet \Phi .
$$

\subsection{Quantum Lie algebra of diffeomorphisms}

By applying the twist deformation to the Lie algebra of vector fields $\operatorname{Vec}(\mathcal{M})$ on phase space $\mathcal{M}$, we obtain the quantum Lie algebra of nonassociative diffeomorphisms described in [5]. Again we deform the usual Lie bracket of vector fields to the star-bracket

$$
[u, v]_{\star}=\left[\overline{\mathrm{f}}^{\alpha}(u), \overline{\mathrm{f}}_{\alpha}(v)\right] .
$$


Defining the star-product between elements in $U \operatorname{Vec}(\mathcal{M})$ as $\xi \star \zeta:=\overline{\mathrm{f}}^{\alpha}(\xi) \overline{\mathrm{f}}_{\alpha}(\zeta)$, the starbracket equals the deformed commutator

$$
[u, v]_{\star}=u \star v-{ }^{\alpha} v \star u_{\alpha} .
$$

This deformed Lie bracket satisfies the star-antisymmetry property

$$
[u, v]_{\star}=-\left[{ }^{\alpha} v,{ }_{\alpha} u\right]_{\star}
$$

and the star-Jacobi identity

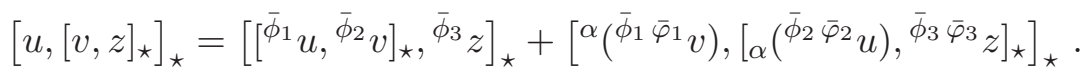

The star-bracket $[,]_{\star}$ makes $\mathrm{Vec}_{\star}$ into the quantum Lie algebra of vector fields.

To implement the action of nonassociative diffeomorphisms on generic differential forms and tensor fields, we need a suitable definition of star-Lie derivative along a vector $u \in V c_{\star}$. From [5] it is a deformation of the ordinary Lie derivative on phase space $\mathcal{M}$ given by

$$
\mathcal{L}_{u}^{\star}(T)=\mathcal{L}_{\overline{\mathrm{f}} \alpha(u)}\left(\overline{\mathrm{f}}_{\alpha}(T)\right)=\mathcal{L}_{\mathrm{D}(u)}(T),
$$

where we introduced the invertible linear map $\mathrm{D}$ on the vector space $U \operatorname{Vec}(\mathcal{M})$ by

$$
\begin{aligned}
\mathrm{D}: U \operatorname{Vec}(\mathcal{M}) & \longrightarrow U \operatorname{Vec}(\mathcal{M}), \\
\xi & \longmapsto \mathrm{D}(\xi):=\overline{\mathrm{f}}^{\alpha}(\xi) \overline{\mathrm{f}}_{\alpha} .
\end{aligned}
$$

With this definition it follows immediately that $\mathcal{L}_{u}^{\star}(v)=[u, v]_{\star}$ for $u, v \in \mathrm{Vec}_{\star}$. Moreover, using the inverse of $(2.9)$ shows that $\mathcal{L}_{\mathrm{D}(\xi)} \bullet \mathcal{L}_{\mathrm{D}(\zeta)}=\mathcal{L}_{\mathrm{D}(\xi \star \zeta)}$, for all $\xi, \zeta \in U \operatorname{Vec}(\mathcal{M})$, so that

$$
\left[\mathcal{L}_{u}^{\star}, \mathcal{L}_{v}^{\star}\right]_{\bullet}=\mathcal{L}_{[u, v]_{\star}}^{\star} .
$$

Thus the star-Lie derivatives provide a representation of the quantum Lie algebra of vector fields on differential forms and tensor fields.

Using (2.9) together with $\Delta(u)=u \otimes 1+1 \otimes u$, the twisted coproducts of $\mathrm{D}(u) \in$ $U \operatorname{Vec}(\mathcal{M})$ are given by

$$
\Delta_{\mathcal{F}}(\mathrm{D}(u))=\mathrm{D}\left(\bar{\phi}_{1} u\right) \bar{\phi}_{2} \otimes \bar{\phi}_{3}+\overline{\mathrm{R}}^{\alpha} \bar{\phi}_{1} \bar{\varphi}_{1} \otimes \mathrm{D}\left(\overline{\mathrm{R}}_{\alpha}\left(\bar{\phi}_{2} \bar{\varphi}_{2} u\right)\right) \bar{\phi}_{3} \bar{\varphi}_{3} .
$$

Using the Leibniz rule for the undeformed Lie derivative $\mathcal{L}_{u}(\omega \wedge \eta)=\mathcal{L}_{u}(\omega) \wedge \eta+\omega \wedge \mathcal{L}_{u}(\eta)$, it follows from (3.68) that the star-Lie derivatives satisfy the deformed Leibniz rule [5]

$$
\mathcal{L}_{u}^{\star}\left(\omega \wedge_{\star} \eta\right)=\mathcal{L}_{\bar{\phi}_{1} u}^{\star}\left({ }^{\phi_{2}} \omega\right) \wedge_{\star}{ }^{\bar{\phi}_{3}} \eta+{ }^{\alpha}\left(\bar{\phi}_{1} \bar{\varphi}_{1} \omega\right) \wedge_{\star} \mathcal{L}_{\alpha}^{\star}\left(\bar{\phi}_{2} \bar{\varphi}_{2} u\right)\left({ }^{\bar{\phi}_{3} \bar{\varphi}_{3}} \eta\right)
$$

on forms $\omega, \eta \in \Omega_{\star}^{\sharp}$. The Leibniz rule for tensor fields is then obtained by replacing differential forms with tensor fields and the deformed exterior product $\wedge_{\star}$ with the deformed tensor product $\otimes_{\star}$. In particular, since $[u, v \star f]_{\star}=\mathcal{L}_{u}^{\star}(v \star f)=\mathcal{L}_{\mathrm{D}(u)}(v \star f)$ for $f \in A_{\star}$, we analogously obtain the Leibniz rule for the quantum Lie bracket of vector fields:

$$
[u, v \star f]_{\star}=\left[\bar{\phi}_{1} u, \bar{\phi}_{2} v\right]_{\star}{ }^{\bar{\phi}_{3}} f+{ }^{\alpha}\left(\bar{\phi}_{1} \bar{\varphi}_{1} v\right) \star \mathcal{L}_{\alpha}^{\star}\left(\bar{\phi}_{2} \bar{\varphi}_{2} u\right)\left(\bar{\phi}_{3} \bar{\varphi}_{3} f\right) .
$$

Since the map D is invertible, as in the noncommutative and associative case $[2,6]$, the symmetry properties of the quasi-Hopf algebra of infinitesimal diffeomorphisms $U \operatorname{Vec}^{\mathcal{F}}(\mathcal{M})$ are equivalently encoded in the quantum Lie algebra of diffeomorphisms $V_{e} c_{\star}$ with bracket $[,]_{\star}$, or in its universal enveloping algebra generated by sums of star-products of elements in $\mathrm{Vec}_{\star}$. 


\section{Nonassociative differential geometry}

\subsection{Connections}

A star-connection is a linear map

$$
\begin{aligned}
\nabla^{\star}: \operatorname{Vec}_{\star} & \longrightarrow \operatorname{Vec}_{\star} \otimes_{\star} \Omega_{\star}^{1} \\
u & \longmapsto \nabla^{\star} u=u^{i} \otimes_{\star} \omega_{i},
\end{aligned}
$$

where $u^{i} \otimes_{\star} \omega_{i} \in \operatorname{Vec}_{\star} \otimes_{\star} \Omega_{\star}^{1}$, which satisfies the right Leibniz rule

$$
\nabla^{\star}(u \star f)=\left({ }^{\bar{\phi}_{1}} \nabla^{\star}\left(\bar{\phi}_{2} u\right)\right) \star^{\bar{\phi}_{3}} f+u \otimes_{\star} \mathrm{d} f
$$

for $u \in \operatorname{Vec}_{\star}$ and $f \in A_{\star}$. The action of $\phi_{a}$ on $\nabla^{\star}$ is the adjoint action (3.35), which in the present instance is readily seen to also define a connection. For this, we calculate

$$
\begin{aligned}
& \phi_{a} \nabla^{\star}(u \star f)={ }^{\phi_{a(1)}}\left(\nabla^{\star}\left({ }^{S\left(\phi_{a_{(2)}}\right)}{ }_{(1)} u \star{ }^{\left.S\left(\phi_{a_{(2)}}{ }{ }^{2}\right) f\right)}\right)\right.
\end{aligned}
$$

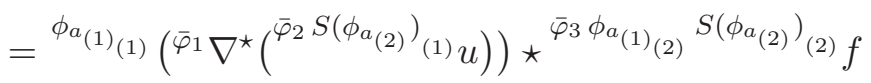

$$
\begin{aligned}
& +{ }_{a_{(1)}(1)} S\left(\phi_{a_{(2)}}\right)_{(1)} u \otimes_{\star}{ }^{\phi_{a_{(1)}(2)}} S\left(\phi_{a_{(2)}}\right)_{(2)} \mathrm{d} f \\
& =\left(\bar{\varphi}_{1}\left(\phi_{a} \nabla^{\star}\right)\left(\bar{\varphi}^{\bar{\varphi}_{2}} u\right)\right) \star^{\bar{\varphi}_{3}} f+{ }^{\phi_{a(1)}} S\left(\phi_{a_{(2)}}\right)\left(u \otimes_{\star} \mathrm{d} f\right) \\
& =\left(\bar{\varphi}_{1}\left(\phi_{a} \nabla^{\star}\right)\left(\bar{\varphi}_{2} u\right)\right) \star^{\bar{\varphi}_{3}} f+\epsilon\left(\phi_{a}\right) u \otimes_{\star} \mathrm{d} f,
\end{aligned}
$$

where in the last line we used (2.15). Now since ${ }^{\phi_{a}} \nabla^{\star}$ will always appear in linear combinations with the other associator legs $\phi_{b}$ and $\phi_{c}$, and since $\epsilon\left(\phi_{a}\right) \phi_{b} \otimes \phi_{c}=1 \otimes 1$, we effectively have the Leibniz rule

$$
{ }^{\phi_{a}} \nabla^{\star}(u \star f)=\left({ }^{\bar{\varphi}_{1}}\left({ }^{\phi_{a}} \nabla^{\star}\right)\left(\bar{\varphi}_{2} u\right)\right) \star^{\bar{\varphi}_{3}} f+u \otimes_{\star} \mathrm{d} f .
$$

More generally, the adjoint action of an element $\xi \in U \operatorname{Vec}^{\mathcal{F}}(\mathcal{M})$ gives the linear map ${ }^{\xi} \nabla^{\star}$ : $\mathrm{Vec}_{\star} \rightarrow \mathrm{Vec}_{\star} \otimes_{\star} \Omega_{\star}^{1}$ which satisfies ${ }^{\xi} \nabla^{\star}(u \star f)=\left(\bar{\phi}_{1} \xi \nabla^{\star}\left(\bar{\phi}_{2} u\right)\right){ }_{\star} \bar{\phi}_{3} f+u \otimes_{\star} \epsilon(\xi) \mathrm{d} f$, i.e., ${ }^{\xi} \nabla^{\star}$ is a connection with respect to the rescaled exterior derivative ${ }^{\xi} \mathrm{d}=\mathcal{L}_{\xi_{(1)}} \mathrm{d} \mathcal{L}_{S\left(\xi_{(2)}\right)}=\epsilon(\xi) \mathrm{d}$.

The connection on vector fields (4.1) uniquely extends to a covariant derivative

$$
\begin{aligned}
\mathrm{d}_{\nabla^{\star}}: \operatorname{Vec}_{\star}^{\sharp} & \longrightarrow \operatorname{Vec}_{\star}^{\sharp+1} \\
u \otimes_{\star} \omega & \longmapsto\left({ }^{\bar{\phi}_{1}} \nabla^{\star}\left(\bar{\phi}_{2} u\right)\right) \wedge_{\star} \bar{\phi}_{3} \omega+u \otimes_{\star} \mathrm{d} \omega
\end{aligned}
$$

on vector fields valued in the exterior algebra $\operatorname{Vec}_{\star}^{\sharp}=\operatorname{Vec}_{\star} \otimes_{\star} \Omega_{\star}^{\sharp}$. It satisfies the graded right Leibniz rule

$$
\mathrm{d}_{\nabla^{\star}}\left(\psi \wedge_{\star} \omega\right)=\left(\bar{\phi}_{1} \mathrm{~d}_{\nabla^{\star}}\left(\bar{\phi}_{2} \psi\right)\right) \wedge_{\star} \bar{\phi}_{3} \omega+(-1)^{|\psi|} \psi \wedge_{\star} \mathrm{d} \omega
$$

for $\psi=u^{i} \otimes_{\star} \omega_{i} \in \operatorname{Vec}_{\star}^{\sharp}$.

The covariant derivative along a vector field $v \in V_{e} c_{\star}$ is defined via the pairing operator as

$$
\nabla_{v}^{\star} u=\left\langle\nabla^{\star} u, v\right\rangle_{\star}=\left\langle\left(u^{i} \otimes_{\star} \omega_{i}\right), v\right\rangle_{\star}={ }^{\phi_{1}} u^{i} \star\left\langle{ }^{\phi_{2}} \omega_{i},{ }^{\phi_{3}} v\right\rangle_{\star} .
$$


From the definition of the pairing (3.27), the Leibniz rule for $\nabla_{v}^{\star}$ comes in the somewhat complicated form that we will need later:

$$
\begin{aligned}
& \nabla_{v}^{\star}(u \star f)=\left\langle\nabla^{\star}(u \star f), v\right\rangle_{\star} \\
&=\left\langle\left(\bar{\phi}_{1} \nabla^{\star}\left(\bar{\phi}_{2} u\right) \star \bar{\phi}_{3} f\right)+\left(u \otimes_{\star} \mathrm{d} f\right), v\right\rangle_{\star} \\
&=\left\langle{ }^{\phi_{1}}\left(\bar{\phi}_{1} \nabla^{\star}\left(\bar{\phi}_{2} u\right)\right) \star\left({ }^{\phi_{2} \bar{\phi}_{3}} f,{ }^{\phi_{3}} v\right)\right\rangle_{\star}+{ }^{\phi_{1}} u \star\left\langle\left({ }^{\phi_{2}}(\mathrm{~d} f),{ }^{\phi_{3}} v\right)\right\rangle_{\star} \\
&=\left\langle{ }^{\phi_{1}}\left(\bar{\phi}_{1} \nabla^{\star}\left(\bar{\phi}_{2} u\right)\right),\left({ }^{\alpha}\left({ }^{\phi_{3}} v\right)\right\rangle_{\star} \star \alpha\right. \\
&\left.\left.\phi^{\phi_{2}} \bar{\phi}_{3} f\right)\right)+{ }^{\phi_{1}} u \star\left\langle{ }^{\phi_{2}}(\mathrm{~d} f),{ }^{\phi_{3}} v\right\rangle_{\star} \\
&=\left(\left\langle\bar{\varphi}_{1} \phi_{1}\left(\bar{\phi}_{1} \nabla^{\star}\left(\bar{\phi}_{2} u\right)\right), \bar{\varphi}_{2}\left({ }^{\alpha}\left({ }^{\phi_{3}} v\right)\right\rangle_{\star}\right) \star{ }^{\bar{\varphi}_{3}}\left({ }_{\alpha}\left({ }^{\phi_{2}} \bar{\phi}_{3} f\right)\right)+{ }^{\phi_{1}} u \star\left\langle\mathrm{d}\left({ }^{\phi_{2}} f\right),{ }^{\phi_{3}} v\right\rangle_{\star} .\right.
\end{aligned}
$$

More generally, we define

$$
\mathrm{d}_{\nabla_{v}^{\star}} \psi:=\left\langle\mathrm{d}_{\nabla_{\star}} \psi, v\right\rangle_{\star}+\mathrm{d}_{\nabla_{\star}}\langle\psi, v\rangle_{\star}
$$

for $\psi=u^{i} \otimes_{\star} \omega_{i} \in \operatorname{Vec}_{\star}^{\sharp}$.

The action of the connection on the basis vectors defines the connection coefficients $\Gamma_{A C}^{B} \in A_{\star}$ through

$$
\nabla^{\star} \partial_{A}=: \partial_{B} \otimes_{\star} \Gamma_{A}^{B}=: \partial_{B} \otimes_{\star}\left(\Gamma_{A C}^{B} \star \mathrm{d} x^{C}\right)
$$

Then we have

$$
\begin{aligned}
\nabla_{A}^{\star} \partial_{B} & :=\left\langle\nabla^{\star} \partial_{B}, \partial_{A}\right\rangle_{\star} \\
& =\left\langle\left(\partial_{C} \otimes_{\star}\left(\Gamma_{B D}^{C} \star \mathrm{d} x^{D}\right)\right), \partial_{A}\right\rangle_{\star} \\
& =\left\langle\left(\partial_{C} \star \Gamma_{B D}^{C}\right) \otimes_{\star} \mathrm{d} x^{D}, \partial_{A}\right\rangle_{\star} \\
& ={ }^{\phi_{1}}\left(\partial_{C} \star \Gamma_{B D}^{C}\right) \star\left\langle{ }^{\phi_{2}} \mathrm{~d} x^{D},{ }^{\phi_{3}} \partial_{A}\right\rangle_{\star} \\
& =\partial_{C} \star \Gamma_{B A}^{C},
\end{aligned}
$$

where we used the definition (3.32), and the contributions from nonassociativity vanish because we used basis vector fields and basis 1-forms. Using the Leibniz rule (4.2) and writing an arbitrary vector field $u$ as $u=\partial_{A} \star u^{A}$ with $u^{A} \in A_{\star}$ one can calculate

$$
\nabla^{\star} u=\partial_{A} \otimes_{\star}\left(\mathrm{d} u^{A}+\Gamma_{B}^{A} \star u^{B}\right)
$$

and more generally

$$
\mathrm{d}_{\nabla^{\star}}\left(\partial_{A} \otimes_{\star} \omega^{A}\right)=\partial_{A} \otimes_{\star}\left(\mathrm{d} \omega^{A}+\Gamma_{B}^{A} \wedge_{\star} \omega^{B}\right)
$$

for $\omega^{A} \in \Omega_{\star}^{\sharp}$.

\subsection{Dual connections}

By considering 1-forms as dual to vector fields, we can define the dual connection ${ }^{\star} \nabla$ on 1 -forms in terms of the connection on vector fields and the exterior derivative as

$$
\left\langle{ }^{\star} \nabla \omega, u\right\rangle_{\star}=\mathrm{d}\langle\omega, u\rangle_{\star}-\left\langle{ }^{\phi_{1}} \omega,{ }^{\phi_{2}} \nabla^{\star}\left({ }^{\phi_{3}} u\right)\right\rangle_{\star} .
$$


Since the pairing is nondegenerate, this defines a connection on the dual bimodule

$$
{ }^{\star} \nabla: \Omega_{\star}^{1} \longrightarrow \Omega_{\star}^{1} \otimes_{\star} \Omega_{\star}^{1} .
$$

This connection acts from the right so that we should more properly write $(\omega)^{\star} \nabla$ rather than ${ }^{\star} \nabla(\omega)$, but this notation is awkward so we refrain from using it. That the action is from the right immediately follows by comparing the $U \mathrm{Vec}^{\mathcal{F}}(\mathcal{M})$-equivariance property (3.36) of the evaluation from the left with the $U \mathrm{Vec}^{\mathcal{F}}(\mathcal{M})$-equivariance property of the evaluation of ${ }^{\star} \nabla$ on $\omega,{ }^{\xi}\left({ }^{\star} \nabla \omega\right)={ }^{\xi}(2){ }^{\star} \nabla\left({ }^{\xi_{(1)}} \omega\right)$, which shows that evaluation is from the right so that the equivariance reads $\xi\left((\omega)^{\star} \nabla\right)=\left(\xi_{(1)} \omega\right)^{\xi_{(2)}} \nabla$. The proof follows from

$$
\begin{aligned}
\left.\xi^{\xi} \nabla \omega, u\right\rangle_{\star} & =\mathrm{d}\left\langle{ }^{\xi_{(1)}} \omega,{ }^{\xi_{(2)}} u\right\rangle_{\star}-\left\langle{ }^{\xi_{(1)} \phi_{1}} \omega,{ }^{\xi_{(2)}(1)}{ }^{\phi_{2}} \nabla^{\star}\left({ }^{\xi_{(2)}(2)}{ }^{\phi_{3}} u\right)\right\rangle_{\star} \\
& =\mathrm{d}\left\langle\xi_{(1)} \omega, \xi_{(2)} u\right\rangle_{\star}-\left\langle{ }^{\phi_{1} \xi_{(1)}(1) \omega},{ }^{\phi_{2} \xi_{(1)}(2)} \nabla^{\star}\left({ }^{\phi_{3} \xi_{(2)}} u\right)\right\rangle_{\star} \\
& =\left\langle{ }^{\xi_{(1)}(2)}{ }^{\star} \nabla\left({ }^{\xi_{(1)}(1)} \omega\right),{ }^{\left.\xi_{(2)} u\right\rangle_{\star},}\right.
\end{aligned}
$$

where in the last line we used $\left\langle{ }^{\xi} \nabla \omega, u\right\rangle_{\star}=\epsilon(\xi) \mathrm{d}\langle\omega, u\rangle_{\star}-\left\langle{ }^{\phi_{1}} \omega,{ }^{\phi_{2}}{ }^{\xi} \nabla^{\star}\left({ }^{\phi_{3}} u\right)\right\rangle_{\star}$, which is easily understood by recalling that $\xi^{\xi} \nabla^{\star}$ is a connection with respect to the rescaled exterior derivative ${ }^{\xi} \mathrm{d}=\epsilon(\xi) \mathrm{d}$.

Correspondingly, the connection ${ }^{\star} \nabla$ satisfies the left Leibniz rule

$$
{ }^{\star} \nabla(f \star \omega)={ }^{\phi_{1}} f \star\left({ }^{\phi_{3} \star} \nabla\left({ }^{\phi_{2}} \omega\right)\right)+\mathrm{d} f \otimes_{\star} \omega
$$

for $f \in A_{\star}$ and $\omega \in \Omega_{\star}^{1}$. The proof follows from the definition (4.14) and the right Leibniz rule for $\nabla^{\star}$ after some associator gymnastics. It uniquely lifts to a connection

$$
\mathrm{d}_{\star} \nabla: \Omega_{\star}^{\sharp} \otimes_{\star} \Omega_{\star}^{1} \longrightarrow \Omega_{\star}^{\sharp+1} \otimes_{\star} \Omega_{\star}^{1} .
$$

Setting $\omega=\mathrm{d} x^{A}$ and $u=\partial_{B}$ in (4.14), so that $\mathrm{d}\left\langle\mathrm{d} x^{A}, \partial_{B}\right\rangle_{\star}=\mathrm{d} \delta^{A}{ }_{B}=0$, we compute

$$
\begin{aligned}
\left\langle{ }^{\star} \nabla\left(\mathrm{d} x^{A}\right), \partial_{B}\right\rangle_{\star} & =-\left\langle\mathrm{d} x^{A}, \nabla^{\star} \partial_{B}\right\rangle_{\star} \\
& =-\left\langle\mathrm{d} x^{A}, \partial_{C} \otimes_{\star} \Gamma_{B}^{C}\right\rangle_{\star} \\
& =-\Gamma_{B}^{A}
\end{aligned}
$$

so that ${ }^{\star} \nabla\left(\mathrm{d} x^{A}\right)=-\Gamma_{B}^{A} \otimes_{\star} \mathrm{d} x^{B}$. Then for $\omega=\omega_{A} \star \mathrm{d} x^{A} \in \Omega_{\star}^{1}$ with $\omega_{A} \in A_{\star}$ we have

$$
\begin{aligned}
{ }^{\star} \nabla \omega & ={ }^{\star} \nabla\left(\omega_{A} \star \mathrm{d} x^{A}\right) \\
& =\omega_{A} \star{ }^{\star} \nabla\left(\mathrm{d} x^{A}\right)+\mathrm{d} \omega_{A} \otimes_{\star} \mathrm{d} x^{A} \\
& =-\omega_{A} \star\left(\Gamma_{B}^{A} \otimes_{\star} \mathrm{d} x^{B}\right)+\mathrm{d} \omega_{A} \otimes_{\star} \mathrm{d} x^{A} \\
& =\left(\mathrm{d} \omega_{B}-\omega_{A} \star \Gamma_{B}^{A}\right) \otimes_{\star} \mathrm{d} x^{B} .
\end{aligned}
$$

More generally, for $\omega_{A} \in \Omega_{\star}^{\sharp}$ we have

$$
\mathrm{d}_{\star} \nabla\left(\omega_{A} \otimes_{\star} \mathrm{d} x^{A}\right)=\left(\mathrm{d} \omega_{A}-\omega_{B} \wedge_{\star} \Gamma_{A}^{B}\right) \otimes_{\star} \mathrm{d} x^{A} .
$$

These results are natural nonassociative generalizations of the usual results in noncommutative differential geometry, since the associator acts trivially on the basis vector fields and basis 1-forms. 


\subsection{Connections on tensor products}

Later on we shall need to compute the action of connections on metric tensors, for which we require a construction of connections on tensor products of $A_{\star}$-bimodules. The general construction is an extension to the nonassociative case of the noncommutative construction in [4] and is provided in ([11], section 4.2). Here we shall give a somewhat simpler and more explicit treatment. Given $A_{\star}$-bimodules $V_{\star}$ and $W_{\star}$, together with connections $\nabla_{V_{\star}}^{\star}$ : $V_{\star} \rightarrow V_{\star} \otimes_{\star} \Omega_{\star}^{1}$ and $\nabla_{W_{\star}}^{\star}: W_{\star} \rightarrow W_{\star} \otimes_{\star} \Omega_{\star}^{1}$, we wish to construct a connection $\nabla_{V_{\star} \otimes_{\star} W_{\star}}:=$ $\nabla_{V_{\star}}^{\star} \oplus_{\star} \nabla_{W_{\star}}^{\star}:\left(V_{\star} \otimes_{\star} W_{\star}\right) \rightarrow\left(V_{\star} \otimes_{\star} W_{\star}\right) \otimes_{\star} \Omega_{\star}^{1}$. Using (3.58) we define

$$
\nabla_{V_{\star}}^{\star} \oplus_{\star} \nabla_{W_{\star}}^{\star}=\nabla_{V_{\star}}^{\star} \otimes \mathrm{id}+\mathrm{id} \otimes_{\mathcal{R}} \nabla_{W_{\star}}^{\star} .
$$

Explicitly, using (3.48) and (3.58) we have

$$
\nabla_{V_{\star} \otimes_{\star} W_{\star}}\left(v \otimes_{\star} w\right)={ }^{\bar{\phi}_{1}} \nabla_{V_{\star}}^{\star}\left(\bar{\phi}_{2} v\right) \otimes_{\star} \bar{\phi}_{3} w+{ }^{\beta \bar{\phi}_{3}} \alpha v \otimes_{\star} \beta{ }^{\bar{\phi}_{1}} \nabla_{W_{\star}}^{\star}\left(\bar{\phi}_{2} \alpha w\right),
$$

where we identify $\left(V_{\star} \otimes_{\star} \Omega_{\star}^{1}\right) \otimes_{\star} W_{\star} \cong\left(V_{\star} \otimes_{\star} W_{\star}\right) \otimes_{\star} \Omega_{\star}^{1}$ via $\left(v \otimes_{\star} \omega\right) \otimes_{\star} w=\left({ }^{\bar{\phi}} 1 v \otimes_{\star} \bar{\phi}_{2} \alpha w\right) \otimes_{\star}$ $\bar{\phi}_{3}{ }_{\alpha} \omega$ for $v \in V_{\star}, w \in W_{\star}$ and $\omega \in \Omega_{\star}^{1}$.

From the general analysis of section 3.6 it follows that this definition is equivariant:

$$
\xi\left(\nabla_{V_{\star}}^{\star} \oplus_{\star} \nabla_{W_{\star}}^{\star}\right)={ }^{\xi} \nabla_{V_{\star}}^{\star} \oplus_{\star}^{\xi} \nabla_{W_{\star}}^{\star}
$$

for any $\xi \in U \operatorname{Vec}^{\mathcal{F}}(\mathcal{M})$. Next we need to check that this definition is well-defined:

$$
\left(\nabla_{V_{\star}}^{\star} \oplus_{\star} \nabla_{W_{\star}}^{\star}\right)\left((v \star f) \otimes_{\star} w\right)=\left(\nabla_{V_{\star}}^{\star} \oplus_{\star} \nabla_{W_{\star}}^{\star}\right)\left({ }^{\rho_{1}} v \otimes_{\star}\left({ }^{\rho_{2}} f \star{ }^{\rho_{3}} w\right)\right) .
$$

Again by the general analysis of section 3.6, we know that this identity holds if the starconnection $\nabla^{\star}$ is substituted by a right $A_{\star}$-linear map $L$, i.e., it holds for the terms which come from the right $A_{\star}$-linear part of the Leibniz rule for $\nabla^{\star}$, so we only need to check the inhomogeneous terms coming from the exterior derivative: on the left-hand side this comes from the application of $\nabla_{V_{\star}}^{\star} \otimes$ id to $(v \star f) \otimes_{\star} w$ which gives $\left(v \otimes_{\star} \mathrm{d} f\right) \otimes_{\star} w$ on using the fact that ${ }^{\phi_{a}} \nabla_{V_{\star}}^{\star}$ is also a connection, whereas on the right-hand side it comes from applying $\tau_{\mathcal{R}} \bullet\left(\nabla_{W_{\star}}^{\star} \otimes \mathrm{id}\right)$ to $\left({ }^{\beta \alpha_{(2)} \rho_{3}} w \star \beta^{\alpha_{(1)} \rho_{2}} f\right) \otimes_{\star} \alpha^{\rho_{1}} v$ which on using the $\mathcal{R}$-matrix identities (2.17) and (2.19) yields

$$
\tau_{\mathcal{R}}\left({ }^{\alpha}\left({ }^{\rho_{2}} \mathrm{~d} f \otimes_{\star}{ }^{\rho_{3}} w\right) \otimes_{\star}{ }^{\rho_{1}} v\right)={ }_{\alpha}{ }^{\rho_{1}} v \otimes_{\star}{ }^{\alpha}\left({ }^{\rho_{2}} \mathrm{~d} f \otimes_{\star}{ }^{\rho_{3}} w\right)=\left(v \otimes_{\star} \mathrm{d} f\right) \otimes_{\star} w
$$

as required. Finally, we show that the map $\nabla_{V_{\star}}^{\star} \oplus_{\star} \nabla_{W_{\star}}^{\star}$ is a connection because it satisfies the Leibniz rule:

$$
\left(\nabla_{V_{\star}}^{\star} \oplus_{\star} \nabla_{W_{\star}}^{\star}\right)\left(\left(v \otimes_{\star} w\right) \star f\right)=\left(\bar{\phi}_{1}\left(\nabla_{V_{\star}}^{\star} \oplus_{\star} \nabla_{W_{\star}}^{\star}\right)\left(\bar{\phi}_{2}\left(v \otimes_{\star} w\right)\right)\right){ }^{\bar{\phi}_{3}} f+\left(v \otimes_{\star} w\right) \otimes_{\star} \mathrm{d} f .
$$

Again it suffices to check the inhomogeneous term, which comes from (id $\left.\otimes_{\mathcal{R}} \nabla_{W_{\star}}^{\star}\right)\left(\left(v \otimes_{\star}\right.\right.$ $w) \star f)$, and the result follows by a completely analogous calculation to (4.26).

We can iterate the twisted sum of connections to arbitrary numbers of tensors products. The nonassociativity of $\oplus_{\star}$ is controlled in the usual way by suitable insertions of the associator [12]:

$$
\left(\nabla_{V_{\star}}^{\star} \oplus_{\star} \nabla_{W_{\star}}^{\star}\right) \oplus_{\star} \nabla_{X_{\star}}^{\star}=\Phi^{-1} \bullet\left(\nabla_{V_{\star}}^{\star} \oplus_{\star}\left(\nabla_{W_{\star}}^{\star} \oplus_{\star} \nabla_{X_{\star}}^{\star}\right)\right) \bullet \Phi .
$$




\subsection{Torsion}

In order to define the torsion $\mathrm{T}^{\star} \in \mathrm{Vec}_{\star} \otimes_{\star} \Omega_{\star}^{2}$ of a connection $\nabla^{\star}$, we first observe that the map

$$
\left\langle\partial_{A} \otimes_{\star} \mathrm{d} x^{A},\right\rangle_{\star}: \mathrm{Vec}_{\star} \longrightarrow \mathrm{Vec}_{\star}
$$

is the identity map; for this, we simply expand any vector field $u$ as $u=\partial_{A} \star u^{A}$, and use the triviality of the associator when acting on $\partial_{A}$ and $\mathrm{d} x^{A}$. Then as in the classical case we define

$$
\begin{aligned}
\mathrm{T}^{\star} & :=\mathrm{d}_{\nabla^{\star}}\left(\partial_{A} \otimes_{\star} \mathrm{d} x^{A}\right) \\
& =\nabla^{\star} \partial_{A} \wedge_{\star} \mathrm{d} x^{A} \\
& =\partial_{B} \otimes_{\star}\left(\Gamma_{A C}^{B} \star\left(\mathrm{d} x^{C} \wedge_{\star} \mathrm{d} x^{A}\right)\right) \\
& =: \partial_{A} \otimes_{\star} \mathrm{T}^{A} .
\end{aligned}
$$

We can also regard the torsion in the usual way as a map $T^{\star}: \mathrm{Vec}_{\star} \otimes_{\star} \mathrm{Vec}_{\star} \rightarrow \mathrm{Vec}_{\star}$ defined by

$$
\mathrm{T}^{\star}(u, v)=\left\langle\mathrm{T}^{\star}, u \otimes_{\star} v\right\rangle_{\star},
$$

where $\left\langle\mathrm{T}^{\star}, u \otimes_{\star} v\right\rangle_{\star}=\partial_{A} \star\left\langle\mathrm{T}^{A}, u \otimes_{\star} v\right\rangle_{\star}$. This map is right $A_{\star}$-linear in its second argument by (3.29), and star-antisymmetric: $\mathrm{T}^{\star}(u, v)=-\mathrm{T}^{\star}\left({ }^{\alpha} v,{ }_{\alpha} u\right)$. This follows form

$$
\left\langle\mathrm{d} x^{A} \wedge_{\star} \mathrm{d} x^{B}, u \otimes_{\star} v\right\rangle_{\star}=\left\langle\mathrm{d} x^{A} \otimes_{\star} \mathrm{d} x^{B}, u \wedge_{\star} v\right\rangle_{\star},
$$

i.e., from $\left\langle{ }^{\alpha} \mathrm{d} x^{B} \otimes_{\star} \alpha \mathrm{d} x^{A}, u \otimes_{\star} v\right\rangle_{\star}=\left\langle\mathrm{d} x^{A} \otimes_{\star} \mathrm{d} x^{B},{ }^{\gamma} v \otimes_{\star} \gamma u\right\rangle_{\star}$. To prove this last equality we recall that the associator is trivial if it acts on the basis 1-forms and apply the definition of the pairing; then reordering we obtain the equivalent expression $\left\langle{ }^{\alpha} \mathrm{d} x^{B},{ }^{\beta} v\right\rangle_{\star} \star$ $\left\langle\beta_{(1)} \alpha \mathrm{d} x^{A}, \beta_{(2)} u\right\rangle_{\star}=\left\langle{ }^{\alpha_{(1)}} \mathrm{d} x^{B},{ }^{\alpha_{(2)} \gamma} v\right\rangle_{\star} \star\left\langle{ }_{\alpha} \mathrm{d} x^{A},{ }_{\gamma} u\right\rangle_{\star}$, which follows from (2.18) and (2.19). Thus we have shown that the map $T^{\star}$ is the torsion tensor $T^{\star} \in \operatorname{hom}_{\star}\left(\operatorname{Vec}_{\star} \wedge_{\star}\right.$ $\left.\mathrm{Vec}_{\star}, \mathrm{Vec}_{\star}\right)$.

In our good basis one easily calculates the torsion components from (4.30) and obtains

$$
\mathrm{T}^{\star}\left(\partial_{A}, \partial_{B}\right)=\partial_{C} \star\left\langle\mathrm{T}^{C}, \partial_{A} \otimes_{\star} \partial_{B}\right\rangle_{\star}=\partial_{C} \star\left(\Gamma_{A B}^{C}-\Gamma_{B A}^{C}\right)=: \partial_{C} \star \mathrm{T}^{C}{ }_{A B} .
$$

The torsion-free condition $\mathbf{T}^{\star}\left(\partial_{A}, \partial_{B}\right)=0$ then results in the symmetric connection coefficients

$$
\Gamma_{A B}^{C}=\Gamma_{B A}^{C} .
$$

We shall now prove the first Cartan structure equation, which in the present context states that the torsion tensor (4.31) can be written in terms of covariant derivatives as

$$
\mathrm{T}^{\star}(u, v)={ }^{\phi_{1}} \nabla_{\phi_{2} v}^{\star}\left({ }^{\phi_{3}} u\right)-{ }^{\phi_{1}} \nabla_{\phi_{\phi_{\alpha}} u}^{\star}\left({ }^{\phi_{3} \alpha} v\right)+[u, v]_{\star}
$$


The expression (4.35) agrees with the definition of torsion from [14]. To prove (4.35), we first check it in our good basis: we set $u=\partial_{A}, v=\partial_{B}$ and easily calculate

$$
\begin{aligned}
& \nabla_{B}^{\star} \partial_{A}-\nabla_{\alpha}^{\star} \partial_{A} \alpha \partial_{B}+\left[\partial_{A}, \partial_{B}\right]_{\star}=\nabla_{B}^{\star} \partial_{A}-\nabla_{A}^{\star} \partial_{B} \\
& =\partial_{C} \star\left(\Gamma_{A B}^{C}-\Gamma_{B A}^{C}\right) \\
& =\mathrm{T}^{\star}\left(\partial_{A}, \partial_{B}\right) \text {, }
\end{aligned}
$$

where we used $\left[\partial_{A}, \partial_{B}\right]_{\star}=0$. The equality (4.35) then follows once we establish that the right-hand side defines a tensor in $\operatorname{hom}_{\star}\left(\mathrm{Vec}_{\star} \wedge_{\star} \mathrm{Vec}_{\star}, \mathrm{Vec}_{\star}\right)$.

For this, it is useful to write the right-hand side of (4.35) as $\boldsymbol{T}^{\star}(u, v)$, where

$$
T^{\star}:=\langle\rangle_{\star} \bullet\left(\nabla^{\star} \otimes \mathrm{id}\right)-\langle\rangle_{\star} \bullet\left(\nabla^{\star} \otimes \mathrm{id}\right) \bullet \tau_{\mathcal{R}}+[\quad]_{\star} .
$$

Here we used the fact that the compositon $\bullet$ is associative since the pairing and the braiding are $U \operatorname{Vec}^{\mathcal{F}}(\mathcal{M})$-equivariant; this implies that the composition $\bullet$ in this case reduces to the usual composition of operators. The associators entering (4.35) are then due to the definition $\left(\nabla^{\star} \otimes\right.$ id $)\left(u \otimes_{\star} v\right)=\bar{\phi}_{1} \nabla^{\star}\left(\bar{\phi}_{2}(u)\right) \otimes_{\star} \bar{\phi}_{3} v$ from $(3.48)$.

As defined in (4.37), the map $\boldsymbol{T}^{\star}$ is linear in both of its arguments because it is a composition of linear maps. A first step in showing that $T^{\star}$ defines a tensor in $\operatorname{hom}_{\star}\left(\operatorname{Vec}_{\star} \wedge_{\star}\right.$ $\left.V e c_{\star}, V e c_{\star}\right)$ is showing that it is well-defined on $V e c_{\star} \otimes_{\star} V e c_{\star}$ :

$$
\boldsymbol{T}^{\star}(u \star f, v)=\boldsymbol{T}^{\star}\left({ }^{\phi_{1}} u,{ }^{\phi_{2}} f \star{ }^{\phi_{3}} v\right)
$$

for all $f \in A_{\star}$ and $u, v \in \operatorname{Vec}_{\star}$, so that we can write $\boldsymbol{T}^{\star}(u, v)=\boldsymbol{T}^{\star}\left(u \otimes_{\star} v\right)$. Explicitly, as before with the sum of connections, we know that (4.38) holds for the terms which come from the right $A_{\star}$-linear part of the Leibniz rule, so we only need to check that the inhomogeneous terms coming from the exterior derivative cancel out. In $\boldsymbol{T}^{\star}(u \star f, v)$ such terms come from ${ }^{\phi_{1}} \nabla_{\phi_{2 v}}^{\star}\left({ }^{\phi_{3}}(u \star f)\right)$, which yields $\left\langle\left(u \otimes_{\star} \mathrm{d} f\right), v\right\rangle_{\star}$, and from

$$
\begin{aligned}
& {[u \star f, v]_{\star}=-\left[{ }^{\alpha} v,{ }_{\alpha}(u \star f)\right]_{\star}} \\
& =-\left[{ }^{\phi_{1} \varphi_{1} \rho_{1} \alpha \beta} v,{ }^{\phi_{2} \varphi_{2} \rho_{2}}{ }_{\alpha} u \star{ }^{\phi_{3} \varphi_{3} \rho_{3}}{ }_{\beta} f\right]_{\star}
\end{aligned}
$$

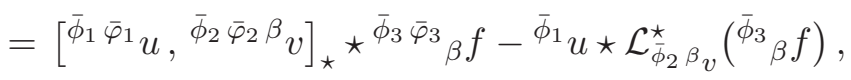

where we used (2.19) and (3.70). By definition of the Lie derivative we have

$$
\mathcal{L}_{u}^{\star}(f)=\mathcal{L}_{\overline{\mathrm{f}} \alpha(u)}\left(\overline{\mathrm{f}}_{\alpha}(f)\right)=\left\langle\overline{\mathrm{f}}^{\alpha}(u), \overline{\mathrm{f}}_{\alpha}(\mathrm{d} f)\right\rangle=\left\langle{ }^{\beta} \mathrm{d} f,{ }_{\beta} u\right\rangle_{\star},
$$

and so $\mathcal{L}_{\bar{\phi}_{2} \beta_{v}}^{\star}\left(\bar{\phi}_{3} \beta f\right)=\left\langle\bar{\phi}_{3} \mathrm{~d} f, \bar{\phi}_{2} v\right\rangle_{\star}$. It follows that the inhomogeneous term in (4.39) can be written as

$$
\bar{\phi}_{1} u \star \mathcal{L}_{\bar{\phi}_{2} \beta}^{\star}\left(\bar{\phi}_{3} \beta f\right)=\left\langle\left(\bar{\varphi}_{1} \phi_{1} u \otimes_{\star} \bar{\varphi}_{2} \phi_{2} \mathrm{~d} f\right), \bar{\varphi}_{3} \phi_{3} v\right\rangle_{\star}=\left\langle\left(u \otimes_{\star} \mathrm{d} f\right), v\right\rangle_{\star}
$$

and hence cancels the appropriate term. Next, it follows immediately that $\boldsymbol{T}^{\star}$ restricts from $V e c_{\star} \otimes_{\star} V e c_{\star}$ to $V e c_{\star} \wedge_{\star} V e c_{\star}$ because it is star-antisymmetric under exchange of its arguments. Finally, we need to check right $A_{\star}$-linearity

$$
\boldsymbol{T}^{\star}\left(\left(u \otimes_{\star} v\right) \star f\right)={ }^{\bar{\phi}_{1}} \boldsymbol{T}^{\star}\left(\bar{\phi}_{2}\left(u \otimes_{\star} v\right)\right) \star{ }^{\bar{\phi}_{3}} f,
$$


which is equivalent to

$$
\boldsymbol{T}^{\star}\left(\phi_{1} u,{ }^{\phi_{2}} v \star{ }^{\phi_{3}} f\right)={ }^{\bar{\phi}_{1} \bar{\varphi}_{1}} \boldsymbol{T}^{\star}\left(\bar{\phi}_{2} u, \bar{\varphi}_{2} v\right) \star{ }^{\bar{\phi}_{3} \bar{\varphi}_{3}} f
$$

Again we just check that the inhomogeneous terms coming from the Leibniz rule cancel out: the contribution to the left-hand side from the first covariant derivative in (4.35) is right $A_{\star}$-linear by the same computation that led to (3.55), while by (2.18) the star-Lie derivative term from (3.70) is cancelled by the inhomogeneous term from

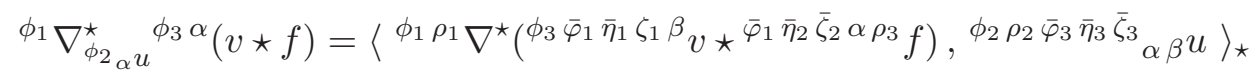

after using (4.40) and (4.8).

\subsection{Curvature}

We proceed by defining the curvature of a connection as in the classical case, i.e., as the square of the covariant derivative, with the composition being the $U \operatorname{Vec}^{\mathcal{F}}(\mathcal{M})$-equivariant --composition of linear maps:

$$
\mathrm{R}^{\star}:=\mathrm{d}_{\nabla^{\star}} \bullet \mathrm{d}_{\nabla^{\star}}: \operatorname{Vec}_{\star} \longrightarrow \operatorname{Vec}_{\star} \otimes_{\star} \Omega_{\star}^{2} .
$$

This definition is well-posed because the linear map $\mathrm{d}_{\nabla^{\star}} \bullet \mathrm{d}_{\nabla^{\star}}$ is right $A_{\star}$-linear and hence defines a tensor $\mathrm{d}_{\nabla^{\star}} \bullet \mathrm{d}_{\nabla^{\star}} \in \operatorname{hom}_{\star}\left(V_{\star}, V_{\star} \otimes_{\star} \Omega_{\star}^{2}\right)$. Right $A_{\star}$-linearity is proven by repeated iteration of the Leibniz rule for $\nabla^{\star}$, giving

$$
\begin{aligned}
& \left(\mathrm{d}_{\nabla^{\star}} \bullet \mathrm{d}_{\nabla^{\star}}\right)(v \star f)={ }^{\phi_{1} \varphi_{1}} \mathrm{~d}_{\nabla^{\star}}\left({ }^{\phi_{2} \varphi_{2}} \nabla^{\star}\left({ }^{\phi_{3}} v \star{ }^{\varphi_{3}} f\right)\right) \\
& ={ }^{\phi_{1} \varphi_{1}} \mathrm{~d}_{\nabla^{\star}}\left({ }^{\phi_{2} \varphi_{2}}\left[{ }^{\bar{\rho}_{1}} \nabla^{\star}\left(\bar{\rho}_{2} \phi_{3} v\right) \star{ }^{\bar{\rho}_{3} \varphi_{3}} f\right]\right)+{ }^{\phi_{1} \varphi_{1}} \mathrm{~d}_{\nabla^{\star}}\left({ }^{\phi_{2} \varphi_{2}}\left({ }^{\phi_{3}} v \otimes_{\star} \mathrm{d}^{\varphi_{3}} f\right)\right) \\
& ={ }^{\phi_{1} \check{\phi}_{1} \varphi_{1}} \mathrm{~d}_{\nabla^{\star}}\left(\phi_{2} \varphi_{2} \bar{\rho}_{1} \nabla^{\star}\left(\bar{\rho}_{2} \phi_{3} \check{\phi}_{3} v\right) \star \check{\phi}_{2} \bar{\rho}_{3} \varphi_{3} f\right)+{ }^{\phi_{1} \varphi_{1}} \mathrm{~d}_{\nabla^{\star}}\left(\varphi_{2} \phi_{3} v \otimes_{\star} \mathrm{d}^{\phi_{2} \varphi_{3}} f\right) \\
& ={ }^{\phi_{1} \check{\phi}_{1} \varphi_{1}} \mathrm{~d}_{\nabla^{\star}}\left(\phi_{2} \varphi_{2} \bar{\rho}_{1} \nabla^{\star}\left(\bar{\rho}_{2} \phi_{3} \check{\phi}_{3} v\right)\right) \star \check{\phi}_{2} \bar{\rho}_{3} \varphi_{3} f+\mathrm{d}_{\nabla^{\star}}\left(v \otimes_{\star} \mathrm{d} f\right) \\
& \text { - }{ }_{1} \check{\phi}_{1} \varphi_{1}\left(\phi_{2} \varphi_{2} \bar{\rho}_{1} \nabla^{\star}\left(\bar{\rho}_{2} \check{\phi}_{3} v\right) \otimes_{\star} \check{\phi}_{2} \bar{\rho}_{3} \varphi_{3}(\mathrm{~d} f)\right) \\
& =\bar{\phi}_{1}\left(\mathrm{~d}_{\nabla^{\star}} \bullet \mathrm{d}_{\nabla^{\star}}\right)\left(\bar{\phi}_{2} v\right) \star \bar{\phi}_{3} f+\mathrm{d}_{\nabla^{\star}}\left(v \otimes_{\star} \mathrm{d} f\right) \\
& -\check{\phi}_{1} \varphi_{1} \phi_{2} \varphi_{2} \bar{\rho}_{1} \nabla^{\star}\left(\bar{\rho}_{2} \phi_{3} \check{\phi}_{3} v\right) \otimes_{\star} \phi_{1} \check{\phi}_{2} \bar{\rho}_{3} \varphi_{3}(\mathrm{~d} f) \\
& =\bar{\phi}_{1}\left(\mathrm{~d}_{\nabla^{\star}} \bullet \mathrm{d}_{\nabla^{\star}}\right)\left(\bar{\phi}_{2} v\right) \star \bar{\phi}_{3} f+\mathrm{d}_{\nabla^{\star}}\left(v \otimes_{\star} \mathrm{d} f\right)-\bar{\rho}^{\bar{\rho}_{1}} \nabla^{\star}\left(\bar{\rho}_{2} v\right) \otimes_{\star} \bar{\rho}_{3}(\mathrm{~d} f) \\
& =\bar{\phi}_{1}\left(\mathrm{~d}_{\nabla^{\star}} \bullet \mathrm{d}_{\nabla^{\star}}\right)\left(\bar{\phi}_{2} v\right) \star{ }^{\bar{\phi}_{3}} f \text {. }
\end{aligned}
$$

For trivial associator this definition of curvature reduces to the noncommutative curvature considered in [4], while the general noncommutative and nonassociative curvature defined in [11] requires an extra braided commutator in the setting of arbitrary quasi-Hopf algebras. 
Acting on a basis vector field $\partial_{A}$ gives

$$
\begin{aligned}
\mathrm{R}^{\star}\left(\partial_{A}\right) & ={ }^{\phi_{1}} \mathrm{~d}_{\nabla^{\star}}\left({ }^{\phi_{2}} \nabla^{\star}\left({ }^{\phi_{3}} \partial_{A}\right)\right) \\
& =\mathrm{d}_{\nabla^{\star}}\left(\nabla^{\star} \partial_{A}\right) \\
& =\mathrm{d}_{\nabla^{\star}}\left(\partial_{B} \otimes_{\star} \Gamma_{A}^{B}\right) \\
& =\bar{\phi}_{1} \nabla^{\star}\left(\bar{\phi}_{2} \partial_{B}\right) \wedge_{\star} \bar{\phi}_{3} \Gamma_{A}^{B}+\partial_{B} \otimes_{\star} \mathrm{d} \Gamma_{A}^{B} \\
& =\left(\nabla^{\star} \partial_{B}\right) \wedge_{\star} \Gamma_{A}^{B}+\partial_{B} \otimes_{\star} \mathrm{d} \Gamma_{A}^{B} \\
& =\left(\partial_{C} \otimes_{\star} \Gamma_{B}^{C}\right) \wedge_{\star} \Gamma_{A}^{B}+\partial_{C} \otimes_{\star} \mathrm{d} \Gamma_{A}^{C} \\
& ={ }^{\phi_{1}} \partial_{C} \otimes_{\star}\left({ }^{\phi_{2}} \Gamma_{B}^{C} \wedge_{\star}{ }^{\phi_{3}} \Gamma_{A}^{B}\right)+\partial_{C} \otimes_{\star} \mathrm{d} \Gamma_{A}^{C} \\
& =\partial_{C} \otimes_{\star}\left(\Gamma_{B}^{C} \wedge_{\star} \Gamma_{A}^{B}\right)+\partial_{C} \otimes_{\star} \mathrm{d} \Gamma_{A}^{C} \\
& =\partial_{C} \otimes_{\star}\left(\mathrm{d} \Gamma_{A}^{C}+\Gamma_{B}^{C} \wedge_{\star} \Gamma_{A}^{B}\right) \\
& =: \partial_{C} \otimes_{\star} \mathrm{R}_{A}^{C} .
\end{aligned}
$$

We used the fact that the associator acts trivially on basis vector fields and that the covariant derivative on form-valued vector fields acts on the form-valued part just as the exterior derivative $\mathrm{d}$ (see (4.5)), which commutes with vector fields (in particular those defining the twist (2.1)). Taking the exterior derivative of the torsion 2-form from (4.30) yields the first Bianchi identity

$$
\mathrm{d}^{A}+\Gamma_{B}^{A} \wedge_{\star} \mathrm{T}^{B}=\mathrm{R}_{B}^{A} \wedge_{\star} \mathrm{d} x^{B},
$$

whereas taking the exterior derivative of the curvature 2-form from (4.47) gives the second Bianchi identity which reads

$$
\mathrm{dR}_{A}^{C}+\Gamma_{B}^{C} \wedge_{\star} \mathrm{R}_{A}^{B}-\mathrm{R}_{B}^{C} \wedge_{\star} \Gamma_{A}^{B}=\Gamma_{B}^{C} \wedge_{\star}\left(\Gamma_{D}^{B} \wedge_{\star} \Gamma_{A}^{D}\right)-{ }^{\phi_{1}} \Gamma_{B}^{C} \wedge_{\star}\left({ }^{\phi_{2}} \Gamma_{D}^{B} \wedge_{\star}{ }^{\phi_{3}} \Gamma_{A}^{D}\right) .
$$

We see that the naive expression for the second Bianchi identity is modified by the associator of connection 1-forms. In the associative case, the right-hand side vanishes and one recovers the usual expression of the second Bianchi identity.

Similarly to the torsion, we can also regard the curvature as the tensor field $\mathrm{R}^{\star} \in$ $\operatorname{hom}_{\star}\left(\operatorname{Vec}_{\star} \otimes_{\star}\left(\operatorname{Vec}_{\star} \wedge_{\star} \operatorname{Vec}_{\star}\right), \operatorname{Vec}_{\star}\right)$ given on vectors $u, v, z \in \operatorname{Vec}_{\star}$ by the vector field

$$
\mathrm{R}^{\star}(z, u, v):=\left\langle\bar{\phi}_{1} \mathrm{R}^{\star}\left(\bar{\phi}_{2} z\right), \bar{\phi}_{3}\left(u \otimes_{\star} v\right)\right\rangle_{\star} .
$$

Indeed from the definition we see that $\mathrm{R}^{\star}(z, u, v)=\mathrm{R}^{\star}\left(z, u \otimes_{\star} v\right)$, and moreover it is not difficult to show that it gives the same result when evaluated on $\left(z \star f, u \otimes_{\star} v\right)$ and on $\left(\phi_{1} z, \phi_{2} f \star \phi_{3}(u \otimes v)\right)$ so that it is well-defined on $\operatorname{Vec}_{\star} \otimes_{\star}\left(\operatorname{Vec}_{\star} \otimes_{\star} \operatorname{Vec}_{\star}\right)$. Hence we can write

$$
\mathrm{R}^{\star}(z, u, v)=\mathrm{R}^{\star}\left(z \otimes_{\star}\left(u \otimes_{\star} v\right)\right) .
$$

This is also consistent with the $U \operatorname{Vec}^{\mathcal{F}}(\mathcal{M})$-action:

$$
\xi\left(\mathrm{R}^{\star}\left(z \otimes_{\star}\left(u \otimes_{\star} v\right)\right)\right)=\xi_{(1)} \mathrm{R}^{\star}\left(\xi_{(2)}\left(z \otimes_{\star}\left(u \otimes_{\star} v\right)\right)\right),
$$


for all $\xi \in U \operatorname{Vec}^{\mathcal{F}}(\mathcal{M})$; this follows by using (4.50) and $\xi_{(1)}\left(\bar{\phi}_{1} \mathrm{R}^{\star}\left(\bar{\phi}_{2} z\right)\right)=$ $\xi_{(1)}{ }_{(1)} \bar{\phi}_{1} \mathrm{R}^{\star}\left({ }^{\xi_{(1)}(2)} \bar{\phi}_{2} z\right)$, and then the quasi-associativity property (2.12) of the coproduct. Finally, the map $\mathrm{R}^{\star}$ is right $A_{\star}$-linear:

$$
\mathrm{R}^{\star}\left(\left(z \otimes_{\star}\left(u \otimes_{\star} v\right)\right) \star f\right)={ }^{\bar{\phi}_{1}} \mathrm{R}^{\star}\left(\bar{\phi}_{2}\left(z \otimes_{\star}\left(u \otimes_{\star} v\right)\right)\right) \star{ }^{\bar{\phi}_{3}} f
$$

We have thus shown that $\mathrm{R}^{\star} \in \operatorname{hom}_{\star}\left(\mathrm{Vec}_{\star} \otimes_{\star}\left(\mathrm{Vec}_{\star} \otimes_{\star} \mathrm{Vec}_{\star}\right), \mathrm{Vec}_{\star}\right)$; since moreover $\mathrm{R}^{\star}(z, u, v)=-\mathrm{R}^{\star}\left(z,{ }^{\alpha} v,{ }_{\alpha} u\right)$, we conclude that $\mathrm{R}^{\star} \in \operatorname{hom}_{\star}\left(\operatorname{Vec}_{\star} \otimes_{\star}\left(\operatorname{Vec}_{\star} \wedge_{\star} \operatorname{Vec}_{\star}\right), \operatorname{Vec}_{\star}\right)$.

One readily extracts the explicit expression for the curvature coefficients with respect to the good basis $\partial_{A}$. Using the star-pairing and (4.47) we get

$$
\begin{aligned}
\mathrm{R}^{\star}\left(\partial_{A}, \partial_{B}, \partial_{C}\right)= & \left\langle\partial_{D} \otimes_{\star} \mathrm{R}_{A}^{D}, \partial_{B} \wedge_{\star} \partial_{C}\right\rangle_{\star} \\
= & \partial_{D} \star\left\langle\mathrm{d} \Gamma_{A}^{D}+\Gamma_{B^{\prime}}^{D} \wedge_{\star} \Gamma_{A}^{B^{\prime}}, \partial_{B} \wedge_{\star} \partial_{C}\right\rangle_{\star} \\
= & \partial_{D} \star\left\langle\left(\left(\partial_{A^{\prime}} \Gamma_{A E}^{D} \star \mathrm{d} x^{A^{\prime}}\right) \wedge_{\star} \mathrm{d} x^{E}\right.\right. \\
& \left.+\left(\Gamma_{B^{\prime} E}^{D} \star \mathrm{d} x^{E}\right) \wedge_{\star}\left(\Gamma_{A F}^{B^{\prime}} \star \mathrm{d} x^{F}\right), \partial_{B} \wedge_{\star} \partial_{C}\right\rangle_{\star} \\
= & \partial_{D} \star\left\langle\partial_{A^{\prime}} \Gamma_{A E}^{D} \star\left(\mathrm{d} x^{A^{\prime}} \wedge_{\star} \mathrm{d} x^{E}\right)\right. \\
& \left.+\Gamma_{B^{\prime} E}^{D} \star\left(\delta^{E} E^{\prime} \Gamma_{A F}^{B^{\prime}}+\mathrm{i} \kappa \mathscr{R}^{E G}{ }_{E^{\prime}}\left(\partial_{G} \Gamma_{A F}^{B^{\prime}}\right)\right) \star\left(\mathrm{d} x^{E^{\prime}} \wedge_{\star} \mathrm{d} x^{F}\right), \partial_{B} \wedge_{\star} \partial_{C}\right\rangle_{\star} \\
= & \partial_{D} \star\left(\partial_{C} \Gamma_{A B}^{D}-\partial_{B} \Gamma_{A C}^{D}-\Gamma_{B^{\prime} E}^{D} \star\left(\delta^{E}{ }_{B} \Gamma_{A C}^{B^{\prime}}+\mathrm{i} \kappa \mathscr{R}^{E G}{ }_{B}\left(\partial_{G} \Gamma_{A C}^{B^{\prime}}\right)\right)\right. \\
& \left.+\Gamma_{B^{\prime} E}^{D} \star\left(\delta^{E} \Gamma_{C B}^{B_{A}^{\prime}}+\mathrm{i} \kappa \mathscr{R}^{E G}{ }_{C}\left(\partial_{G} \Gamma_{A B}^{B^{\prime}}\right)\right)\right) \\
= & \partial_{D} \star \mathrm{R}^{D}{ }_{A B C},
\end{aligned}
$$

where once again we used the fact that the associator acts trivially on the basis vectors and basis 1-forms.

We shall now prove the second Cartan structure equation, which in the present context states that the curvature tensor (4.50) can be written in terms of covariant derivatives as

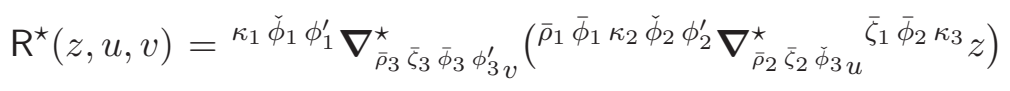

$$
\begin{aligned}
& -{ }^{\kappa_{1} \check{\phi}_{1} \phi_{1}^{\prime}} \nabla_{\bar{\rho}_{3} \bar{\zeta}_{3} \bar{\phi}_{3} \phi_{3 \alpha}^{\prime} u}^{\star}\left(\bar{\rho}_{1} \bar{\phi}_{1} \kappa_{2} \check{\phi}_{2} \phi_{2}^{\prime} \nabla_{\bar{\rho}_{2} \bar{\zeta}_{2} \check{\phi}_{3} \alpha v}^{\star} \bar{\zeta}_{1} \bar{\phi}_{2} \kappa_{3} z\right)+\nabla_{[u, v]_{*}}^{\star} z
\end{aligned}
$$

where to streamline the notation we introduced the bold-face covariant derivative

$$
\nabla_{v}^{\star} u:=\left\langle\bar{\phi}_{1} \nabla^{\star}\left(\bar{\phi}_{2} u\right), \bar{\phi}_{3} v\right\rangle_{\star}
$$

The expression (4.55) for the curvature agrees with that of [14] after taking into account their different conventions; ${ }^{1}$ for trivial associator it reduces to the general expression in [6]. To prove (4.55) we first check it on our good basis by setting $z=\partial_{A}, u=\partial_{B}$ and $v=\partial_{C}$.

\footnotetext{
${ }^{1}$ We are grateful to Michael Fuchs for pointing this out to us.
} 
Then the right-hand side reduces to

$$
\begin{aligned}
\nabla_{C}^{\star} & \left(\nabla_{B}^{\star} \partial_{A}\right)-\nabla_{\alpha}^{\star} \partial_{B}\left(\nabla_{\alpha}^{\star} \partial_{C} \partial_{A}\right)+\nabla_{\left[\partial_{B}, \partial_{C}\right]_{\star} \partial_{A}}^{\star}= \\
= & \nabla_{C}^{\star}\left(\nabla_{B}^{\star} \partial_{A}\right)-\nabla_{B}^{\star}\left(\nabla_{C}^{\star} \partial_{A}\right) \\
= & \nabla_{C}^{\star}\left(\partial_{D} \star \Gamma_{A B}^{D}\right)-\nabla_{B}^{\star}\left(\partial_{D} \star \Gamma_{A C}^{D}\right) \\
= & \left\langle\nabla^{\star} \partial_{D},{ }^{\alpha} \partial_{C}\right\rangle_{\star}{ }_{\alpha} \Gamma_{A B}^{D}+\partial_{D} \star\left\langle\mathrm{d} \Gamma_{A B}^{D}, \partial_{C}\right\rangle_{\star} \\
& -\left\langle\nabla^{\star} \partial_{D},{ }^{\alpha} \partial_{B}\right\rangle_{\star}{ }_{\alpha} \Gamma_{A C}^{D}-\partial_{D} \star\left\langle\mathrm{d} \Gamma_{A C}^{D}, \partial_{B}\right\rangle_{\star} \\
= & \mathrm{R}^{\star}\left(\partial_{A}, \partial_{B}, \partial_{C}\right),
\end{aligned}
$$

where in the third equality we used the Leibniz rule (4.8) while the last equality follows from (4.54). The equality (4.55) for arbitrary vectors then follows once we establish that the right-hand side defines a tensor in $\operatorname{hom}_{\star}\left(\operatorname{Vec}_{\star} \otimes_{\star}\left(\operatorname{Vec}_{\star} \wedge_{\star} \operatorname{Vec}_{\star}\right), \operatorname{Vec}_{\star}\right)$.

For this, as in the case of the torsion, we rewrite the right-hand side of (4.55) as a trilinear map $\boldsymbol{R}^{\star}$ on vectors $z, u$ and $v$, and prove that it is a map in $\operatorname{hom}_{\star}\left(\operatorname{Vec}_{\star} \otimes_{\star}\left(\operatorname{Vec}_{\star} \wedge_{\star}\right.\right.$ $\left.\left.V e c_{\star}\right), V e c_{\star}\right)$. To arrive at the form of $\boldsymbol{R}^{\star}$, for notational clarity we first consider vectors $z, u, v$ on which the associator acts trivially (for example basis vectors $\partial_{A}, \partial_{B}, \partial_{C}$ ). Then we reproduce $\nabla_{v}^{\star}\left(\nabla_{u}^{\star} z\right)$ as the elementary compositions

$$
\begin{aligned}
& z \otimes_{\star}\left(u \otimes_{\star} v\right) \stackrel{\nabla^{\star} \otimes_{\star} \mathrm{id}^{\otimes_{\star} 2}}{\longmapsto} \nabla^{\star} z \otimes_{\star}\left(u \otimes_{\star} v\right) \stackrel{\Phi^{-1}}{\longmapsto}\left(\nabla^{\star} z \otimes_{\star} u\right) \otimes_{\star} v \\
& \stackrel{\langle\rangle_{\star} \otimes_{\star} \text { id }}{\longmapsto} \nabla_{u}^{\star} z \otimes_{\star} v \stackrel{\nabla^{\star} \otimes_{\star} \mathrm{id}}{\longmapsto} \nabla^{\star}\left(\nabla_{u}^{\star} z\right) \otimes_{\star} v \stackrel{\longleftrightarrow\rangle_{\star}}{\longmapsto} \nabla_{v}^{\star}\left(\nabla_{u}^{\star} z\right) .
\end{aligned}
$$

This leads to a definition of $\boldsymbol{R}^{\star}$ written solely in terms of the connection $\nabla^{\star}$, the associator $\Phi^{-1}$, and the equivariant maps studied in section 3 , which reads as

$$
\begin{aligned}
\boldsymbol{R}^{\star}:= & \langle\rangle_{\star} \bullet\left(\nabla^{\star} \otimes \mathrm{id}\right) \bullet\left(\langle\rangle_{\star} \otimes \mathrm{id}\right) \bullet \Phi_{\operatorname{Vec}_{\star} \otimes \Omega_{\star}^{1}, \operatorname{Vec}_{\star}, \operatorname{Vec}_{\star}}^{-1} \bullet\left(\nabla^{\star} \otimes \mathrm{id}^{\otimes 2}\right) \bullet\left(\mathrm{id}^{\otimes 3}-\mathrm{id} \otimes_{\mathcal{R}} \tau_{\mathcal{R}}\right) \\
& +\langle\rangle_{\star} \bullet\left(\nabla^{\star} \otimes \mathrm{id}\right) \bullet\left(\mathrm{id} \otimes_{\mathcal{R}}[]_{\star}\right) .
\end{aligned}
$$

Even though the composition $\bullet$ is nonassociative, there is no ambiguity in this definition because of the equivariance of the maps which are composed and because ${ }^{\phi_{a}} \phi_{b}=0$ (the associator being generated by an abelian subalgebra). For these same reasons, there is the more explicit expression

$$
\begin{aligned}
& \boldsymbol{R}^{\star}=\langle\rangle_{\star} \circ\left({ }^{\phi_{1}} \nabla^{\star} \otimes \mathrm{id}\right) \circ\left(\langle\rangle_{\star} \otimes \mathrm{id}\right) \circ \Phi_{\operatorname{Vec}_{\star} \otimes \Omega_{\star}^{1}, \operatorname{Vec}_{\star}, \operatorname{Vec}_{\star}} \circ\left({ }^{\phi_{2}} \nabla^{\star} \otimes \mathrm{id}^{\otimes 2}\right) \circ \phi_{3} \circ\left(\mathrm{id}^{\otimes 3}-\mathrm{id} \otimes \tau_{\mathcal{R}}\right) \\
& +\langle\rangle_{\star} \circ\left(\nabla^{\star} \otimes \mathrm{id}\right) \circ\left(\mathrm{id} \otimes[]_{\star}\right) \text {. }
\end{aligned}
$$

As sought, explicit evaluation of $\boldsymbol{R}^{\star}$ on $z \otimes_{\star}\left(u \otimes_{\star} v\right)$ gives the right-hand side of (4.55):

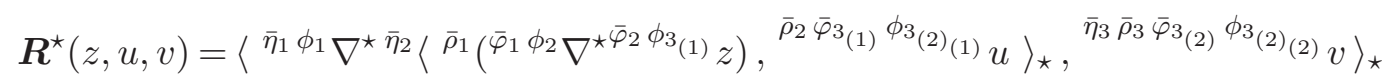

$$
\begin{aligned}
& -\left\langle\bar{\eta}_{1} \phi_{1} \nabla^{\star} \bar{\eta}_{2}\left\langle\bar{\rho}_{1}\left(\bar{\varphi}_{1} \phi_{2} \nabla^{\star \bar{\varphi}_{2} \phi_{3}(1)} z\right),{ }^{\bar{\rho}_{2} \bar{\varphi}_{3(1)} \phi_{3(2)}{ }_{(1)} \alpha} v\right\rangle_{\star},{ }^{\left.\bar{\eta}_{3} \bar{\rho}_{3} \bar{\varphi}_{3(2)}{ }^{\phi_{3}(2)}{ }_{(2)}{ }_{\alpha} u\right\rangle_{\star}}\right. \\
& +\left\langle\bar{\varphi}_{1} \nabla^{\star \bar{\varphi}_{2}} z, \bar{\varphi}_{3}[u, v]_{\star}\right\rangle_{\star}
\end{aligned}
$$

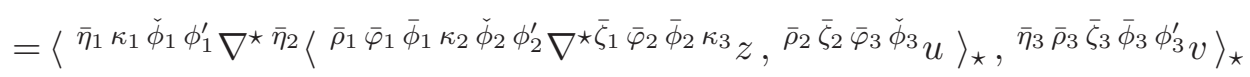

$$
\begin{aligned}
& -\left\langle\bar{\eta}_{1} \kappa_{1} \check{\phi}_{1} \phi_{1}^{\prime} \nabla^{\star \bar{\eta}_{2}}\left\langle\bar{\rho}_{1} \bar{\varphi}_{1} \bar{\phi}_{1} \kappa_{2} \check{\phi}_{2} \phi_{2}^{\prime} \nabla^{\star \bar{\zeta}_{1} \bar{\varphi}_{2} \bar{\phi}_{2} \kappa_{3} z, \bar{\rho}_{2} \bar{\zeta}_{2} \bar{\varphi}_{3} \check{\phi}_{3} \alpha} v\right\rangle_{\star}, \bar{\eta}_{3} \bar{\rho}_{3} \bar{\zeta}_{3} \bar{\phi}_{3} \phi_{3}^{\prime} \alpha u\right\rangle_{\star} \\
& +\left\langle\bar{\varphi}^{\bar{\varphi}_{1}} \nabla^{\star \bar{\varphi}_{2}} z, \bar{\varphi}^{\bar{\varphi}_{3}}[u, v]_{\star}\right\rangle_{\star} .
\end{aligned}
$$


Now the proof that $\boldsymbol{R}^{\star}$ is a map in $\operatorname{hom}_{\star}\left(\operatorname{Vec}_{\star} \otimes_{\star}\left(\operatorname{Vec}_{\star} \wedge_{\star} \operatorname{Vec}_{\star}\right), \operatorname{Vec}_{\star}\right)$ requires as a first step to show that it is a well-defined map on $\operatorname{Vec} c_{\star} \otimes_{\star}\left(\operatorname{Vec}_{\star} \otimes_{\star} V e c_{\star}\right)$ :

$$
\boldsymbol{R}^{\star}(z, u \star f, v)=\boldsymbol{R}^{\star}\left(z,{ }^{\phi_{1}} u,{ }^{\phi_{2}} f \star{ }^{\phi_{3}} v\right),
$$

so that we get a well-defined map $\boldsymbol{R}^{\star}\left(z, u \otimes_{\star} v\right)=\boldsymbol{R}^{\star}(z, u, v)$, and

$$
\boldsymbol{R}^{\star}\left(z \star f, u \otimes_{\star} v\right)=\boldsymbol{R}^{\star}\left({ }^{\phi_{1}} z,{ }^{\phi_{2}} f \star{ }^{\phi_{3}}\left(u \otimes_{\star} v\right)\right),
$$

so that we get a well-defined map $\boldsymbol{R}^{\star}\left(z \otimes_{\star}\left(u \otimes_{\star} v\right)\right)=\boldsymbol{R}^{\star}(z, u, v)$. The star-antisymmetry of $\boldsymbol{R}^{\star}$ under $u \otimes_{\star} v \rightarrow{ }^{\alpha} v \otimes_{\star}{ }_{\alpha} u$ then immediately follows, and this implies that $\boldsymbol{R}^{\star}$ is a linear map from $\operatorname{Vec}_{\star} \otimes_{\star}\left(\mathrm{Vec}_{\star} \wedge_{\star} \mathrm{Vec}_{\star}\right)$ to $\mathrm{Vec}_{\star}$. The final step is to show that $\boldsymbol{R}^{\star} \in$ $\operatorname{hom}_{\star}\left(\operatorname{Vec}_{\star} \otimes_{\star}\left(\operatorname{Vec}_{\star} \wedge_{\star}, \operatorname{Vec}_{\star}\right), \operatorname{Vec}_{\star}\right)$, i.e., that it is right $A_{\star}$-linear:

$$
\boldsymbol{R}^{\star}\left(\left(z \otimes_{\star}\left(u \otimes_{\star} v\right)\right) \star f\right)={ }^{\bar{\phi}_{1}} \boldsymbol{R}^{\star}\left(\bar{\phi}_{2}\left(z \otimes_{\star}\left(u \otimes_{\star} v\right)\right)\right) \star{ }^{\bar{\phi}_{3}} f .
$$

In the following we prove right $A_{\star}$-linearity (4.64); the remaining $A_{\star}$-linearity properties (4.62) and (4.63) can be established with similar techniques.

For this, we note again that if the star-connection $\nabla^{\star}$ and the star-Lie derivative $\mathcal{L}^{\star}=[]_{\star}$ were right $A_{\star}$-linear maps, then the operator (4.59) would also be right $A_{\star^{-}}$ linear because all composite maps would be right $A_{\star}$-linear. Hence as before it suffices to check that the inhomogeneous terms coming from the Leibniz rule for the connection and the Lie derivative cancel out. We denote by Leib* the projector onto the inhomogeneous terms. For example

$$
\operatorname{Leib}^{\star}\left(\nabla^{\star}(u \star f)\right)=u \otimes_{\star} \mathrm{d} f
$$

which induces

$$
\operatorname{Leib}^{\star}\left(\nabla_{v}^{\star}(u \star f)\right)=\left\langle\operatorname{Leib}^{\star}\left(\nabla^{\star}(u \star f)\right), v\right\rangle_{\star}={ }^{\varphi_{1}} u \star\left\langle\varphi^{\varphi_{2}} \mathrm{~d} f,{ }^{\varphi_{3}} v\right\rangle_{\star}=\operatorname{Leib}^{\star}\left(\nabla_{v}^{\star}(u \star f)\right) .
$$

Here we used the fact that in the inhomogeneous term the covariant derivative $\nabla_{v}^{\star}$ from (4.56) acts as a rescaled exterior derivative ${ }^{\bar{\phi}_{1}} \mathrm{~d}=\epsilon\left(\bar{\phi}_{1}\right) \mathrm{d}$, which is $U \operatorname{Vec}^{\mathcal{F}}(\mathcal{M})$ equivariant. Furthermore, from (4.39) we also have

$$
\operatorname{Leib}^{\star}\left([u \star f, v]_{\star}\right)={ }^{\bar{\phi}_{1}} u \star\left\langle{ }^{\bar{\phi}_{3}} \mathrm{~d} f,{ }^{\bar{\phi}_{2}} v\right\rangle_{\star} .
$$

The projector Leib ${ }^{\star}$ in these examples is a linear operator in $u, v$ and $f$. We have to show that $\operatorname{Leib}^{\star}\left(\boldsymbol{R}^{\star}\left(\left(z \otimes_{\star}\left(v \otimes_{\star} u\right)\right) \star f\right)\right)=0$ for all $z, v, u \in \operatorname{Vec}_{\star}$ and $f \in A_{\star}$. Since

$$
\begin{aligned}
\left(z \otimes_{\star}\left(v \otimes_{\star} u\right)\right) \star f & =\varphi_{1} \rho_{1} z \otimes_{\star}\left(\left(\varphi_{2} v \otimes_{\star}{ }^{\rho_{2}} u\right) \star{ }^{\varphi_{3} \rho_{3}} f\right) \\
& =\varphi_{1} \rho_{1} z \otimes_{\star}\left(\zeta_{1} \varphi_{2} v \otimes_{\star}\left({ }^{\zeta_{2} \rho_{2}} u \star{ }^{\zeta_{2} \varphi_{3} \rho_{3}} f\right)\right),
\end{aligned}
$$

this condition is equivalent to $\operatorname{Leib}^{\star}\left(\boldsymbol{R}^{\star}\left(z \otimes_{\star}\left(v \otimes_{\star}(u \star f)\right)\right)\right)=0$ because of linearity in $z, v, u, f$. Hence we check that $\operatorname{Leib}^{\star}\left(\boldsymbol{R}^{\star}(z, v, u \star f)\right)=0$, or equivalently, using starantisymmetry and linearity again, that $\operatorname{Leib}^{\star}\left(\boldsymbol{R}^{\star}(z, u \star f, v)\right)=0$. 
From (4.55) we write

$$
\begin{aligned}
\operatorname{Leib}^{\star}\left(\boldsymbol{R}^{\star}(z, u \star f, v)\right)= & \operatorname{Leib}^{\star}\left(\kappa_{1} \check{\phi}_{1} \phi_{1}^{\prime} \nabla_{\bar{\rho}_{3} \bar{\zeta}_{3} \bar{\phi}_{3} \phi_{3 v}^{\prime}}\left(\bar{\rho}_{1} \bar{\phi}_{1} \kappa_{2} \check{\phi}_{2} \phi_{2}^{\prime} \nabla_{\bar{\rho}_{2} \bar{\zeta}_{2} \check{\phi}_{3}(u \star f)} \bar{\zeta}_{1} \bar{\phi}_{2} \kappa_{3} z\right)\right) \\
& +\operatorname{Leib}^{\star}\left(\nabla_{[u \star f, v]_{\star}}^{\star} z\right)
\end{aligned}
$$

and compare the two contributions. The second contribution in (4.69) is equal to

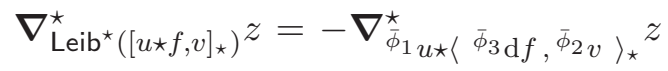

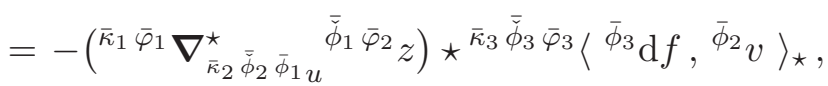

where in the second equality we used the definition (4.56) to rewrite

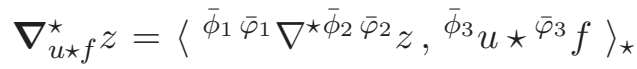

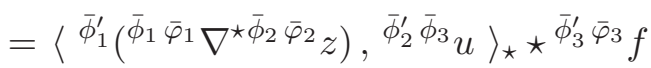

$$
\begin{aligned}
& =\left({ }^{\bar{\kappa}_{1} \bar{\varphi}_{1}} \nabla_{\bar{\kappa}_{2} \bar{\phi}_{2} u} \overline{\bar{\phi}}_{1} \bar{\varphi}_{2} z\right) \star{ }^{\bar{\kappa}_{3} \bar{\phi}_{3} \bar{\varphi}_{3}} f \text {, }
\end{aligned}
$$

and then replace $u \star f$ with ${ }^{\bar{\phi}_{1}} u \star\left\langle\bar{\phi}_{3} \mathrm{~d} f, \bar{\phi}_{2} v\right\rangle_{\star}$. The first contribution in (4.69) can be rewritten without the first three associator legs $\kappa_{a}, \check{\phi}_{a}, \phi_{a}^{\prime}$, because in the inhomoge-

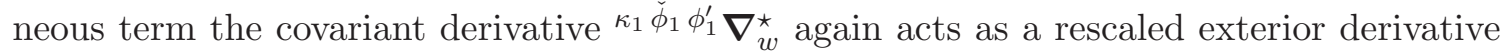
$\kappa_{1} \check{\phi}_{1} \phi_{1}^{\prime} \mathrm{d}=\epsilon\left(\kappa_{1}\right) \epsilon\left(\check{\phi}_{1}\right) \epsilon\left(\phi_{1}^{\prime}\right) \mathrm{d}$, which is $U \operatorname{Vec}^{\mathcal{F}}(\mathcal{M})$-equivariant. Therefore the first contribution in (4.69) equals

$$
\begin{aligned}
& \operatorname{Leib}^{\star}\left(\boldsymbol{\nabla}_{\bar{\rho}_{3} \bar{\phi}_{3}^{\prime} \bar{\zeta}_{3} \bar{\eta}_{3} \bar{\phi}_{3} v}\left(\bar{\rho}_{1} \bar{\phi}_{1}^{\prime} \bar{\phi}_{1} \nabla_{\bar{\rho}_{2} \bar{\zeta}_{2} u \star}^{\star \bar{\phi}_{2}^{\prime} \bar{\eta}_{2} f} \bar{\zeta}_{1} \bar{\eta}_{1} \bar{\phi}_{2} z\right)\right)
\end{aligned}
$$

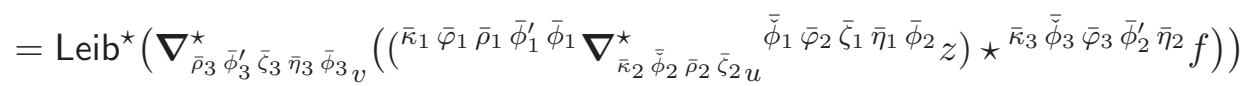

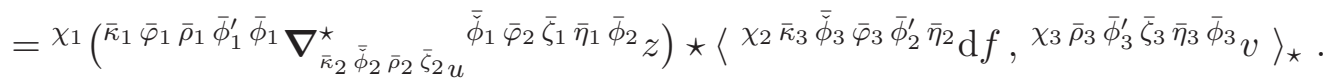

Replacing $(u, f, v)$ with $\left({ }^{\eta_{1}} u, \bar{\eta}_{2} f, \bar{\eta}_{3} v\right)$ in (4.72) gives an action of $\bar{\phi}_{1}^{\prime} \otimes \bar{\eta}_{1} \otimes \bar{\zeta}_{1} \otimes \bar{\phi}_{2}^{\prime} \bar{\zeta}_{2} \bar{\eta}_{2} \otimes$ $\bar{\phi}_{3}^{\prime} \bar{\zeta}_{3} \bar{\eta}_{3}$ which cancels against that of $\left(\left(\Delta_{\mathcal{F}} \otimes \mathrm{id}\right) \Delta_{\mathcal{F}}\left(\chi_{1}\right)\right) \otimes \chi_{2} \otimes \chi_{3}$ and yields

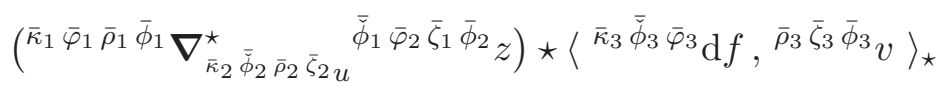

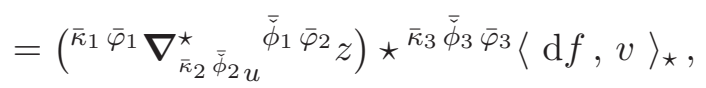

thereby cancelling the contribution (4.70) with the same replacement of $(u, f, v)$. This shows that

$$
\operatorname{Leib}^{\star}\left(\boldsymbol{R}^{\star}\left(z,{ }^{\bar{\eta}_{1}} u \star{ }^{\bar{\eta}_{2}} f,{ }^{\bar{\eta}_{3}} v\right)\right)=0
$$

and hence establishes the right $A_{\star}$-linearity property (4.64) as required. 


\subsection{Ricci tensor}

Since the associator acts trivially on the basis $\mathrm{d} x^{A}$ and its dual $\partial_{A}$, the definition of the Ricci tensor can be given following the noncommutative case studied in [6]. We define

$$
\operatorname{Ric}^{\star}(u, v):=-\left\langle\mathrm{R}^{\star}\left(u, v, \partial_{A}\right), \mathrm{d} x^{A}\right\rangle_{\star},
$$

for all $u, v \in \mathrm{Vec}_{\star}$, where the pairing between a vector field on the left and a form on the right is given by

$$
\langle u, \omega\rangle_{\star}=\left\langle\overline{\mathrm{f}}^{\alpha}(u), \overline{\mathrm{f}}_{\alpha}(\omega)\right\rangle,
$$

similarly to (3.27). The properties of this pairing are completely analogous to those described in section 3.5, by simply interchanging forms and vector fields in all expressions considered there.

We now show that the map (4.75) defines a tensor $\operatorname{Ric}^{\star} \in \operatorname{hom}_{\star}\left(\operatorname{Vec}_{\star} \otimes_{\star} \operatorname{Vec}_{\star}, \operatorname{Vec}_{\star}\right)$. We first prove that it is a map from $\operatorname{Vec}_{\star} \otimes_{\star} \operatorname{Vec}_{\star}$ to $\operatorname{Vec}_{\star}$, so that we can write $\operatorname{Ric}^{\star}(u, v)=$ $\operatorname{Ric}^{\star}\left(u \otimes_{\star} v\right)$; indeed, we have

$$
\begin{aligned}
\operatorname{Ric}^{\star}(u \star f, v) & =-\left\langle\mathrm{R}^{\star}\left(u \star f, v, \partial_{A}\right), \mathrm{d} x^{A}\right\rangle_{\star} \\
& =-\left\langle\mathrm{R}^{\star}\left((u \star f) \otimes_{\star}\left(v \otimes_{\star} \partial_{A}\right)\right), \mathrm{d} x^{A}\right\rangle_{\star} \\
& =-\left\langle\mathrm{R}^{\star}\left({ }^{\phi_{1}} u \otimes_{\star}\left({ }^{\phi_{2}} f \star{ }^{\phi_{3}}\left(v \otimes_{\star} \partial_{A}\right)\right)\right), \mathrm{d} x^{A}\right\rangle_{\star} \\
& =-\left\langle\mathrm{R}^{\star}\left({ }^{\phi_{1}} u \otimes_{\star}\left(\left(^{\phi_{2}} f \star{ }^{\phi_{3}} v\right) \otimes_{\star} \partial_{A}\right)\right), \mathrm{d} x^{A}\right\rangle_{\star} \\
& =\operatorname{Ric}^{\star}\left({ }^{\phi_{1}} u,{ }^{\phi_{2}} f \star{ }^{\phi_{3}} v\right)
\end{aligned}
$$

where in the second equality we used (4.51), and in the fourth equality the fact that the associator acts trivially on $\partial_{A}$. Next we prove compatibility with the $U \operatorname{Vec}^{\mathcal{F}}(\mathcal{M})$-action:

$$
\xi\left(\operatorname{Ric}^{\star}\left(u \otimes_{\star} v\right)\right)=\xi_{(1)} \operatorname{Ric}^{\star}\left(\xi_{(2)}(u \otimes v)\right),
$$

which follows from (4.52), iterated use of (2.12) and from ${ }^{\xi}\left(\partial_{A} \otimes_{\star} \mathrm{d} x^{A}\right)=\epsilon(\xi) \partial_{A} \otimes_{\star} \mathrm{d} x^{A}$; this latter property follows from writing the identity map id : $\mathrm{Vec}_{\star} \rightarrow \mathrm{Vec}_{\star}$ as in (4.29) and using $\xi_{\mathrm{id}}=\epsilon(\xi)$ id, i.e., ${ }^{\xi}(\operatorname{id}(u))=\xi_{(1)} \operatorname{id}\left({ }^{\xi_{(2)}} u\right)=\operatorname{id}\left({ }^{\xi} u\right)$, for all $\xi \in U \operatorname{Vec}^{\mathcal{F}}(\mathcal{M})$ and $u \in \mathrm{Vec}_{\star}$. Finally, we prove right $A_{\star}$-linearity. For this, we notice that

$$
\begin{aligned}
{ }^{\phi_{1}} u \otimes_{\star}\left(\left({ }^{\phi_{2}} v \star{ }^{\phi_{3}} f\right) \otimes_{\star} \partial_{A}\right) & ={ }^{\phi_{1}} u \otimes_{\star}\left({ }^{\phi_{2}} v \otimes_{\star}\left({ }^{\alpha} \partial_{A} \star{ }_{\alpha}{ }^{\phi_{3}} f\right)\right) \\
& ={ }^{\phi_{1}} u \otimes_{\star}\left(\left({ }^{\phi_{2}} v \otimes_{\star}{ }^{\alpha} \partial_{A}\right) \star{ }^{\phi_{3}}{ }_{\alpha} f\right) \\
& =\left(\bar{\varphi}_{1} \phi_{1} u \otimes_{\star} \bar{\varphi}_{2}\left({ }^{\phi_{2}} v \otimes_{\star}{ }^{\alpha} \partial_{A}\right)\right) \star{ }^{\bar{\varphi}_{3} \phi_{3}}{ }_{\alpha} f \\
& =\left(u \otimes_{\star}\left(v \otimes_{\star}{ }^{\alpha} \partial_{A}\right)\right) \star{ }_{\alpha} f
\end{aligned}
$$

where we used the fact that the associator commutes with each leg of the $\mathcal{R}$-matrix and hence it acts trivially on ${ }^{\alpha} \partial_{A}$. Then the proof follows from (4.79) and the centrality of the tensor $\partial_{A} \otimes_{\star} \mathrm{d} x^{A}: f \star\left(\partial_{A} \otimes_{\star} \mathrm{d} x^{A}\right)=\left({ }^{\alpha} \partial_{A} \otimes_{\star}{ }^{\beta} \mathrm{d} x^{A}\right) \star{ }_{\beta \alpha} f=\left(\partial_{A} \otimes_{\star} \mathrm{d} x^{A}\right) \star f$, see (3.16) 
and (3.23), or again (4.29). Explicitly, we have

$$
\begin{aligned}
\operatorname{Ric}^{\star}\left(\left(u \otimes_{\star} v\right) \star f\right) & =\operatorname{Ric}^{\star}\left(\phi_{1} u \otimes_{\star}\left({ }^{\phi_{2}} v \star{ }^{\phi_{3}} f\right)\right) \\
& =-\left\langle\mathrm{R}^{\star}\left({ }^{\phi_{1}} u \otimes_{\star}\left(\left({ }^{\phi_{2}} v \star{ }^{\phi_{3}} f\right) \otimes_{\star} \partial_{A}\right)\right), \mathrm{d} x^{A}\right\rangle_{\star} \\
& =-\left\langle\mathrm{R}^{\star}\left(\left(u \otimes_{\star}\left(v \otimes_{\star}{ }^{\alpha} \partial_{A}\right)\right) \star \alpha f\right), \mathrm{d} x^{A}\right\rangle_{\star} \\
& =-\left\langle\bar{\varphi}_{1} \mathrm{R}^{\star}\left(\bar{\varphi}_{2}\left(u \otimes_{\star}\left(v \otimes_{\star}{ }^{\alpha} \partial_{A}\right)\right)\right) \star{ }^{\bar{\varphi}_{3}}{ }_{\alpha} f, \mathrm{~d} x^{A}\right\rangle_{\star} \\
& =-\left\langle\bar{\varphi}_{1}\left(\mathrm{R}^{\star}\left(\bar{\varphi}_{2}\left(u \otimes_{\star}\left(v \otimes_{\star}{ }^{\alpha} \partial_{A}\right)\right)\right)\right) \star{ }^{\bar{\varphi}_{3}}{ }_{\alpha} f, \mathrm{~d} x^{A}\right\rangle_{\star} \\
& =-\left\langle\bar{\varphi}_{1} \bar{\rho}_{1}\left(\mathrm{R}^{\star}\left(\bar{\varphi}_{2} u \otimes_{\star}\left(\bar{\rho}_{2} v \otimes_{\star}{ }^{\alpha} \partial_{A}\right)\right)\right),{ }^{\bar{\varphi}_{3} \bar{\rho}_{3}}{ }_{\alpha} f \star \mathrm{d} x^{A}\right\rangle_{\star} \\
& =-\left\langle\bar{\varphi}_{1} \bar{\rho}_{1}\left(\mathrm{R}^{\star}\left(\bar{\varphi}_{2} u \otimes_{\star}\left(\bar{\rho}_{2} v \otimes_{\star}{ }^{\alpha} \partial_{A}\right)\right)\right),{ }^{\beta} \mathrm{d} x^{A}\right\rangle_{\star} \star \beta \alpha^{\bar{\varphi}_{3} \bar{\rho}_{3}} f \\
& =-{ }^{\bar{\varphi}_{1} \bar{\rho}_{1}}\left\langle\mathrm{R}^{\star}\left(\bar{\varphi}_{2} u \otimes_{\star}\left(\bar{\rho}_{2} v \otimes_{\star} \partial_{A}\right)\right), \mathrm{d} x^{A}\right\rangle_{\star}{ }^{\bar{\varphi}_{3} \bar{\rho}_{3} f} \\
& =\bar{\varphi}_{1} \bar{\rho}_{1} \operatorname{Ric}^{\star}\left(\bar{\varphi}_{2} u \otimes_{\star} \bar{\rho}_{2} v\right) \star \bar{\varphi}_{3} \bar{\rho}_{3} f \\
& =\bar{\varphi}_{1} \operatorname{Ric}^{\star}\left(\bar{\varphi}_{2}\left(u \otimes_{\star} v\right)\right) \star{ }^{\bar{\varphi}_{3}} f .
\end{aligned}
$$

The coefficients of the Ricci tensor in the coordinate basis are given by

$$
\operatorname{Ric}^{\star}=\operatorname{Ric}_{A D} \star\left(\mathrm{d} x^{D} \otimes_{\star} \mathrm{d} x^{A}\right),
$$

where $\operatorname{Ric}_{B C}:=\operatorname{Ric}^{\star}\left(\partial_{B}, \partial_{C}\right)$; indeed we have

$$
\operatorname{Ric}^{\star}\left(\partial_{B}, \partial_{C}\right)=\left\langle\operatorname{Ric}_{A D} \star\left(\mathrm{d} x^{D} \otimes_{\star} \mathrm{d} x^{A}\right), \partial_{B} \otimes_{\star} \partial_{C}\right\rangle_{\star}=\operatorname{Ric}_{B C}
$$

We use (4.54) together with the fact that the associator acts trivially on basis vectors and basis 1 -forms to calculate explicitly

$$
\begin{aligned}
\operatorname{Ric}_{B C}= & -\left\langle\mathrm{R}^{\star}\left(\partial_{B}, \partial_{C}, \partial_{A}\right), \mathrm{d} x^{A}\right\rangle_{\star} \\
= & -\left\langle\partial_{D} \star \mathrm{R}^{D}{ }_{B C A}, \mathrm{~d} x^{A}\right\rangle_{\star} \\
= & -{ }^{\alpha} \mathrm{R}^{D}{ }_{B C A} \star\left\langle{ }_{\alpha} \partial_{D}, \mathrm{~d} x^{A}\right\rangle_{\star} \\
= & \mathrm{R}^{A}{ }_{B A C}-\mathrm{i} \kappa \mathscr{R}^{E F}{ }_{D} \partial_{E} \mathrm{R}^{D}{ }_{B C A} \star\left\langle\partial_{F}, \mathrm{~d} x^{A}\right\rangle_{\star} \\
= & \mathrm{R}^{A}{ }_{B A C}-\mathrm{i} \kappa \mathscr{R}^{E A}{ }_{D} \partial_{E} \mathrm{R}^{D}{ }_{B C A} \\
= & \partial_{A} \Gamma_{B C}^{A}-\partial_{C} \Gamma_{B A}^{A}+\Gamma_{B^{\prime} A}^{A} \star \Gamma_{B C}^{B^{\prime}}-\Gamma_{B^{\prime} C}^{A} \star \Gamma_{B A}^{B^{\prime}} \\
& +\mathrm{i} \kappa \Gamma_{B^{\prime} E}^{A} \star\left(\mathscr{R}^{E G}{ }_{A}\left(\partial_{G} \Gamma_{B C}^{B^{\prime}}\right)-\mathscr{R}^{E G}{ }_{C}\left(\partial_{G} \Gamma_{B A}^{B^{\prime}}\right)\right) \\
& +\mathrm{i} \kappa \mathscr{R}^{E G}{ }_{A} \partial_{G} \partial_{C} \Gamma_{B E}^{A}-\mathrm{i} \kappa \mathscr{R}^{E G}{ }_{A} \partial_{G}\left(\Gamma_{B^{\prime} E}^{A} \star \Gamma_{B C}^{B^{\prime}}-\Gamma_{B^{\prime} C}^{A} \star \Gamma_{B E}^{B^{\prime}}\right) \\
& +\kappa^{2} \mathscr{R}^{A F}{ }_{D}\left(\mathscr{R}^{E G}{ }_{A} \partial_{F}\left(\Gamma_{B^{\prime} E}^{D} \star \partial_{G} \Gamma_{B C}^{B^{\prime}}\right)-\mathscr{R}^{E G}{ }_{C} \partial_{F}\left(\Gamma_{B^{\prime} E}^{D} \star \partial_{G} \Gamma_{B A}^{B^{\prime}}\right)\right) .
\end{aligned}
$$

This calculation did not use symmetry of $\Gamma_{B C}^{A}$, i.e., the torsion-free condition (4.34). Indeed this is the Ricci tensor of an arbitrary affine connection. 


\section{Nonassociative Riemannian geometry and gravity}

\subsection{Metric and torsion-free connection conditions}

We shall now discuss the metric aspects of nonassociative differential geometry and how it can be used to build a theory of nonassociative gravity. For this, notice first of all that star-symmetric tensors are of the form $T \otimes_{\star} T^{\prime}+{ }^{\alpha} T^{\prime} \otimes_{\star \alpha} T$. A metric tensor is an element $\mathrm{g}^{\star} \in \Omega_{\star}^{1} \otimes_{\star} \Omega_{\star}^{1}$ which can be written in the basis $\mathrm{d} x^{A}$ as $\mathrm{g}^{\star}=\mathrm{g}_{A B} \star\left(\mathrm{d} x^{A} \otimes_{\star} \mathrm{d} x^{B}\right)$ with real-valued components $\mathrm{g}_{A B}=\mathrm{g}_{B A} \in A_{\star}$ (the bracketing here is immaterial due to the basis 1-forms). We can regard it as a map $\mathrm{g}^{\star} \in \mathrm{hom}_{\star}\left(\mathrm{Vec}_{\star} \otimes_{\star} \operatorname{Vec}_{\star}, A_{\star}\right)$ which on vector fields $u, v \in \mathrm{Vec}_{\star}$ gives the function

$$
\mathrm{g}^{\star}(u, v)=\left\langle\mathrm{g}^{\star}, u \otimes_{\star} v\right\rangle_{\star} .
$$

It is star-symmetric: $\mathrm{g}^{\star}(u, v)=\mathrm{g}^{\star}\left({ }^{\alpha} v,{ }_{\alpha} u\right)$, and one easily confirms that $\mathrm{g}^{\star}\left(\partial_{A}, \partial_{B}\right)=\mathrm{g}_{A B}$. As usual we assume that $\mathrm{g}^{\star}$ is nondegenerate: $\mathrm{g}^{\star}(u, v)=0$ for all $v \in \mathrm{Vec}_{\star}$ if and only if $u=0$.

Let us now study the metric compatibility condition for a connection $\nabla^{\star}: \mathrm{Vec}_{\star} \rightarrow$ $V_{e c} \otimes_{\star} \Omega_{\star}^{1}$. The connection $\nabla^{\star}$ gives a connection $\nabla^{\star}: V_{e c} \otimes_{\star} V_{e c} \rightarrow\left(V_{e c} \otimes_{\star} \otimes_{\star} \operatorname{Vec}_{\star}\right) \otimes_{\star} \Omega_{\star}^{1}$ on the tensor product $V e c_{\star} \otimes_{\star} V e c_{\star}$, defined as in section 4.3. The space $\Omega_{\star}^{1} \otimes_{\star} \Omega_{\star}^{1}$ is dual to $\mathrm{Vec}_{\star} \otimes_{\star} \mathrm{Vec}_{\star}$ and hence $\nabla^{\star}: \mathrm{Vec}_{\star} \otimes_{\star} \mathrm{Vec}_{\star} \rightarrow\left(\mathrm{Vec}_{\star} \otimes_{\star} \mathrm{Vec}_{\star}\right) \otimes_{\star} \Omega_{\star}^{1}$ induces a dual connection ${ }^{\star} \nabla: \Omega_{\star}^{1} \otimes_{\star} \Omega_{\star}^{1} \rightarrow\left(\Omega_{\star}^{1} \otimes_{\star} \Omega_{\star}^{1}\right) \otimes_{\star} \Omega_{\star}^{1}$ as in (4.14), now with $\omega \in \Omega_{\star}^{1} \otimes_{\star} \Omega_{\star}^{1}$ and $u \in V_{e c} \otimes_{\star} V_{e c}$. We can then state the metric compatibility condition as

$$
{ }^{\star} \nabla \mathrm{g}^{\star}=0 \text {. }
$$

We shall now show that, as in the classical case, this condition together with the torsion-free condition uniquely determine the connection in terms of the metric.

We start by using (4.14) with $\omega=\mathrm{g}^{\star} \in \Omega_{\star}^{1} \otimes_{\star} \Omega_{\star}^{1}$ and $u=\partial_{A} \otimes_{\star} \partial_{B} \in \operatorname{Vec}_{\star} \otimes_{\star} \operatorname{Vec}_{\star}$ to get

$$
\begin{aligned}
\operatorname{dg}_{A B} & =\mathrm{d}\left\langle\mathrm{g}^{\star}, \partial_{A} \otimes_{\star} \partial_{B}\right\rangle_{\star} \\
& =\left\langle{ }^{\star} \nabla \mathrm{g}^{\star}, \partial_{A} \otimes_{\star} \partial_{B}\right\rangle_{\star}+\left\langle{ }^{\phi_{1}} \mathrm{~g}^{\star},{ }^{\phi_{2}} \nabla^{\star}\left(\phi_{3}\left(\partial_{A} \otimes_{\star} \partial_{B}\right)\right)\right\rangle_{\star} \\
& =\left\langle\mathrm{g}^{\star}, \nabla^{\star}\left(\partial_{A} \otimes_{\star} \partial_{B}\right)\right\rangle_{\star},
\end{aligned}
$$

where we used $\mathrm{g}_{A B}=\mathrm{g}_{B A}$ and the fact that the associators act trivially on the basis vectors. We can write this more explicitly as

$$
\begin{aligned}
\operatorname{dg} A B & =\left\langle\mathrm{g}^{\star}, \nabla^{\star} \partial_{A} \otimes_{\star} \partial_{B}+{ }^{\alpha} \partial_{A} \otimes_{\star \alpha} \nabla^{\star} \partial_{B}\right\rangle_{\star} \\
& =\left\langle\mathrm{g}^{\star}, \nabla^{\star} \partial_{A} \otimes_{\star} \partial_{B}+{ }^{\alpha} \partial_{A} \otimes_{\star} \alpha \nabla^{\star}{ }_{\gamma} \partial_{B}\right\rangle_{\star} \\
& =\left\langle\mathrm{g}^{\star}, \nabla^{\star} \partial_{A} \otimes_{\star} \partial_{B}+{ }^{\alpha} \partial_{A} \otimes_{\star \alpha}\left(\nabla^{\star} \partial_{B}\right)\right\rangle_{\star} \\
& =\left\langle\mathrm{g}^{\star},\left(\partial_{C} \otimes_{\star} \Gamma_{A}^{C}\right) \otimes_{\star} \partial_{B}+{ }^{\alpha} \partial_{A} \otimes_{\star \alpha}\left(\partial_{D} \otimes_{\star} \Gamma_{B}^{D}\right)\right\rangle_{\star} \\
& =\left\langle\mathrm{g}^{\star},\left(\partial_{C} \otimes_{\star} \Gamma_{A}^{C}\right) \otimes_{\star} \partial_{B}+{ }^{\alpha} \partial_{A} \otimes_{\star}\left(\partial_{D} \otimes_{\star \alpha} \Gamma_{B}^{D}\right)\right\rangle_{\star} \\
& =\left\langle\mathrm{g}^{\star}, \partial_{C} \otimes_{\star}\left({ }^{\alpha} \partial_{B} \otimes_{\star \alpha} \Gamma_{A}^{C}\right)+{ }^{\alpha} \partial_{A} \otimes_{\star}\left(\partial_{D} \otimes_{\star \alpha} \Gamma_{B}^{D}\right)\right\rangle_{\star}
\end{aligned}
$$




$$
\begin{aligned}
& =\left\langle\mathrm{g}^{\star}, \partial_{C} \otimes_{\star}\left({ }^{\alpha} \partial_{B} \otimes_{\star \alpha} \Gamma_{A}^{C}\right)+\partial_{D} \otimes_{\star}\left({ }^{\alpha} \partial_{A} \otimes_{\star \alpha} \Gamma_{B}^{D}\right)\right\rangle_{\star} \\
& =\left\langle\mathrm{g}^{\star}, \partial_{C} \otimes_{\star}\left({ }^{\alpha} \partial_{B} \otimes_{\star \alpha} \Gamma_{A}^{C}+{ }^{\alpha} \partial_{A} \otimes_{\star \alpha} \Gamma_{B}^{C}\right)\right\rangle_{\star} \\
& =\left\langle\mathrm{g}_{M C} \star \mathrm{d} x^{M},{ }^{\alpha} \partial_{B} \otimes_{\star \alpha} \Gamma_{A}^{C}+{ }^{\alpha} \partial_{A} \otimes_{\star \alpha} \Gamma_{B}^{C}\right\rangle_{\star} \\
& =\mathrm{g}_{M N} \star\left(\left\langle\mathrm{d} x^{M},{ }^{\alpha} \partial_{B}\right\rangle_{\star}{ }_{\alpha} \Gamma_{A}^{N}+\left\langle\mathrm{d} x^{M},{ }^{\alpha} \partial_{A}\right\rangle_{\star}{ }_{\alpha} \Gamma_{B}^{N}\right),
\end{aligned}
$$

where in the first equality we used (4.23) and the fact that the $\mathcal{R}$-matrix acts trivially on a pair of basis vectors, so that ${ }^{\beta} \partial_{A} \otimes_{\star} \partial_{B}=\partial_{A} \otimes_{\star} \partial_{B}$. In the second equality we used $\xi^{\xi}\left(\nabla^{\star} \partial_{B}\right)=\xi_{(1)} \nabla^{\star}\left(\xi_{(2)} \partial_{B}\right)$ (as for all linear maps acting from the left), the coproduct action (2.19) on the $\mathcal{R}$-matrix, and again triviality of the action of $\mathcal{R}$ as well as of the associator on $\partial_{A} \otimes_{\star} \partial_{B}$. In the sixth line we used the fact that ${ }^{\alpha} \partial_{A}$ is again a basis vector and then star-symmetry of the metric.

We similarly calculate

$$
\begin{aligned}
& \left\langle\mathrm{d} x^{M},{ }^{\alpha} \partial_{B}\right\rangle_{\star} \star{ }_{\alpha} \Gamma_{A}^{N}=\left\langle\mathrm{d} x^{M},{ }^{\alpha} \partial_{B}\right\rangle_{\star}{ }{ }_{\alpha}\left(\Gamma_{A C}^{N} \star \mathrm{d} x^{C}\right) \\
& =\left\langle\mathrm{d} x^{M},{ }^{\beta \gamma} \partial_{B}\right\rangle_{\star}{ }_{\beta} \Gamma_{A C}^{N}{ }^{\star} \gamma \mathrm{d} x^{C} \\
& =\left\langle\mathrm{d} x^{M},{ }^{\beta} \partial_{B}\right\rangle_{\star} \star\left({ }_{\beta} \Gamma_{A C}^{N} \star \mathrm{d} x^{C}\right) \\
& =\left(\left\langle\mathrm{d} x^{M},{ }^{\beta} \partial_{B}\right\rangle_{\star} \star{ }_{\beta} \Gamma_{A C}^{N}\right) \star \mathrm{d} x^{C} \\
& =\left\langle\mathrm{d} x^{M}, \Gamma_{A C}^{N} \star \partial_{B}\right\rangle_{\star} \star \mathrm{d} x^{C},
\end{aligned}
$$

where as usual we used again (2.19) together with ${ }^{\alpha} \partial_{A} \star{ }_{\alpha} \mathrm{d} x^{B}=\partial_{A} \star \mathrm{d} x^{B}$ for our cochain twist (2.1). We can finally write

$$
\mathrm{dg}_{A B}=\mathrm{g}_{M N} \star\left\langle\mathrm{d} x^{M}, \Gamma_{A C}^{N} \star \partial_{B}\right\rangle_{\star} \star \mathrm{d} x^{C}+\mathrm{g}_{M N} \star\left\langle\mathrm{d} x^{M}, \Gamma_{B C}^{N} \star \partial_{A}\right\rangle_{\star} \star \mathrm{d} x^{C} .
$$

We now contract the expression (5.6) with $\partial_{D}$ using the star-pairing to obtain

$$
\partial_{D} \mathrm{~g}_{A B}=\left\langle\mathrm{dg} A B, \partial_{D}\right\rangle_{\star}=\mathrm{g}_{M N} \star\left(\left\langle\mathrm{d} x^{M}, \Gamma_{A D}^{N} \star \partial_{B}\right\rangle_{\star}+\left\langle\mathrm{d} x^{M}, \Gamma_{B D}^{N} \star \partial_{A}\right\rangle_{\star}\right) .
$$

We write the expression (5.7) two more times, with the indices $A, B, D$ cyclically permuted, and consider the combination $\partial_{D} \mathrm{~g}_{A B}+\partial_{A} \mathrm{~g}_{B D}-\partial_{B} \mathrm{~g}_{D A}$. Using the fact that the connection coefficients are symmetric for vanishing torsion (see (4.34)), we obtain

$$
\mathrm{g}_{M N} \star\left\langle\mathrm{d} x^{M}, \Gamma_{A D}^{N} \star \partial_{B}\right\rangle_{\star}=\frac{1}{2}\left(\partial_{D} \mathrm{~g}_{A B}+\partial_{A} \mathrm{~g}_{B D}-\partial_{B} \mathrm{~g}_{A D}\right) .
$$

The left hand side equals $\left\langle\mathrm{g}_{M N} \star \mathrm{d} x^{M} \star \Gamma_{A D}^{N}, \partial_{B}\right\rangle_{\star}$, so star-multiplying (5.8) by any $v^{B} \in A_{\star}$ gives

$$
\left\langle\mathrm{g}_{M N} \star \mathrm{d} x^{M} \star \Gamma_{A D}^{N}, v\right\rangle_{\star}=\frac{1}{2}\left(\partial_{D} \mathrm{~g}_{A B}+\partial_{A} \mathrm{~g}_{B D}-\partial_{B} \mathrm{~g}_{A D}\right) \star v^{B},
$$

with $v=\partial_{B} \star v^{B} \in \mathrm{Vec}_{\star}$. Since the vector field $v$ is arbitrary and the star-pairing is nondegenerate, this shows that

$$
\mathrm{g}_{M N} \star \mathrm{d} x^{M} \star \Gamma_{A D}^{N}=\frac{1}{2}\left(\partial_{D} \mathrm{~g}_{A B}+\partial_{A} \mathrm{~g}_{B D}-\partial_{B} \mathrm{~g}_{A D}\right) \star \mathrm{d} x^{B}
$$


Since moreover the metric $\mathrm{g}^{\star}$ is nondegenerate, the expression (5.10) uniquely defines the torsion-free metric compatible connection in the nonassociative case.

It remains to explicitly solve for the connection coefficients $\Gamma_{A D}^{N}$ from (5.10). For this, we use (3.16) to rewrite (5.10) as

$$
\begin{aligned}
\mathrm{d} x^{M} \star \mathrm{G}_{M N} \star \Gamma_{A D}^{N}= & \mathrm{d} x^{M} \star \frac{1}{2}\left(\partial_{D} \mathrm{~g}_{A M}+\partial_{A} \mathrm{~g}_{M D}-\partial_{M} \mathrm{~g}_{A D}\right. \\
& \left.+\mathrm{i} \kappa \mathscr{R}^{E F}{ }_{M}\left(\partial_{E} \partial_{D} \mathrm{~g}_{A F}+\partial_{E} \partial_{A} \mathrm{~g}_{D F}\right)\right),
\end{aligned}
$$

with

$$
\mathrm{G}_{M N}=\mathrm{g}_{M N}+\mathrm{i} \kappa \mathscr{R}^{E F}{ }_{M} \partial_{E} \mathrm{~g}_{N F},
$$

where the associators act trivially due to the basis vector fields and basis 1-forms. The tensor $\mathrm{G}_{M N}$ is nondegenerate but not symmetric; it can be thought of as a realisation the $R$-flux corrected "effective metric" anticipated from the string theory perspective [14]. We then use the star-pairing to contract (5.11) from the left with $\partial_{C}$ and obtain

$$
\mathrm{G}_{C N} \star \Gamma_{A D}^{N}=\frac{1}{2}\left(\partial_{D} \mathbf{g}_{A C}+\partial_{A} \mathbf{g}_{D C}-\partial_{C} \mathbf{g}_{A D}+\mathrm{i} \kappa \mathscr{R}^{E F}\left(\partial_{E} \partial_{D} \mathbf{g}_{A F}+\partial_{E} \partial_{A} \mathbf{g}_{D F}\right)\right)
$$

We are now faced with the problem of extracting the connection coefficients $\Gamma_{A D}^{N}$ from (5.13).

\subsection{Inversion in $A_{\star}$}

Before tackling the matrix equation (5.13), let us consider a simpler problem involving ordinary functions: start from the equation

$$
h \star g=w,
$$

where $h, g, w \in A_{\star}$ are functions, the star-product is given by (3.6), and $h$ is invertible with respect to the usual pointwise product of functions. From (5.14) we would like to uniquely determine $g$ in terms of $h$ and $w$.

Recalling the factorization (2.3) of the cochain twist $\mathcal{F}$, let $*:=\star_{F}$ be the star-product induced by the twist $F$ from (2.4); this is of course just the canonical associative MoyalWeyl star-product on phase space: $f * g=\overline{\mathbf{f}}^{\alpha}(f) \cdot \overline{\mathbf{f}}_{\alpha}(g)$. All the $R$-flux dependence is contained in the twist $F_{R}$ from (2.5). Let $h^{-1}$ be the usual pointwise inverse of the function $h: h^{-1} \cdot h=h \cdot h^{-1}=1$. Then $h$ is also $*$-invertible [7], and we write $h^{*-1}$ for the $*$-inverse function:

$$
h^{*-1} * h=h * h^{*-1}=1 .
$$

It can be expressed explicitly as a power series [7]

$$
h^{*-1}=h^{-1}+\sum_{n=1}^{\infty} h^{-1} *\left(1-h * h^{-1}\right)^{* n},
$$

where, since $\left(1-h * h^{-1}\right)^{* n}$ is of order $O\left(\hbar^{n}\right)$, in order to get $h^{*-1}$ up to order $O\left(\hbar^{n}\right)$ we only have to compute the finite sum $h^{-1}+\sum_{k=1}^{n} h^{-1} *\left(1-h * h^{-1}\right)^{* k}$. This enables one 
to uniquely determine the function $h^{*-1}$ order by order in $\hbar$. If no $R$-flux is present, the equation (5.14) becomes $h * g=w$ which has the unique solution

$$
g=1 * g=\left(h^{*-1} * h\right) * g=h^{*-1} *(h * g)=h^{*-1} * w .
$$

In the absence of $R$-flux, the problem of determining $g$ from the functions $h$ and $w$ has been solved in a very specific way: by determining a function $h^{*-1}$ whose star-product $*$ with $w$ gives $g$. As similarly observed in [14], in general we cannot solve the equation (5.14) in the nonassociative algebra $A_{\star}$ in this way. If a function $h$ is invertible, and hence $*-$ invertible, then one can recursively construct a right $\star$-inverse $h_{\mathrm{r}}^{\star-1} \in A_{\star}$ for $h$ : writing the power series expansion

$$
h_{\mathrm{r}}^{\star-1}=\sum_{n=0}^{\infty} h_{\mathrm{r}}^{\star-1(n)},
$$

where $h_{\mathrm{r}}^{\star-1(n)}$ is of order $O\left(\kappa^{n}\right)$, by using the fact that $h^{*-1}$ is unique one can solve the equation $h \star h_{\mathrm{r}}^{\star-1}=\overline{\mathrm{f}}_{R}^{\alpha}(h) * \overline{\mathrm{f}}_{R \alpha}\left(h^{\star-1}\right)=1$ order by order in $\kappa$ to obtain the iterative solution

$$
\begin{aligned}
& h_{\mathrm{r}}^{\star-1(0)}=h^{*-1}, \\
& h_{\mathrm{r}}^{\star-1(n)}=-h^{*-1} * \sum_{k=0}^{n-1} \overline{\mathrm{f}}_{R}^{n-k}(h) * \overline{\mathrm{f}}_{R n-k}\left(h^{\star-1(k)}\right), \quad n \geq 1,
\end{aligned}
$$

where the terms of order $O\left(\kappa^{n-k}\right)$ of the twist $F_{R}^{-1}$ are denoted $\overline{\mathrm{f}}_{R}^{n-k} \otimes \overline{\mathrm{f}}_{R n-k}$ (with summation understood). Similarly, one can construct a left $\star$-inverse $h_{1}^{\star-1} \in A_{\star}$ such that $h_{1}^{\star-1} \star h=1$. However, in general

$$
h_{\mathrm{r}}^{\star-1}=\left(h_{1}^{\star-1} \star h\right) \star h_{\mathrm{r}}^{\star-1}={ }^{\phi_{1}} h_{1}^{\star-1} \star\left({ }^{\phi_{2}} h \star{ }^{\phi_{3}} h_{\mathrm{r}}^{\star-1}\right) \neq h_{1}^{\star-1}
$$

so that the left and right $\star$-inverses do not generally coincide. Moreover,

$$
g=1 \star g=\left(h_{1}^{\star-1} \star h\right) \star g={ }^{\phi_{1}} h_{1}^{\star-1} \star\left({ }^{\phi_{2}} h \star{ }^{\phi_{3}} g\right)
$$

and so in general the equation (5.14) cannot be solved by using the left $\star$-inverse.

In any nonassociative unital algebra which is alternative, i.e., for which $(a a) b=a(a b)$ and $(b a) a=b(a a)$, for all algebra elements $a$ and $b$, the theory of inverses is identical to that in associative algebras (see e.g. [28]): inverses of elements when they exist are unique, and equations such as (5.14) have unique solutions exactly as in the associative case. However, the nonassociative algebra $A_{\star}$ is not alternative, see e.g. [18, 23], with the violations always being due to explicit dependence on momentum coordinates $p_{\mu}$ of phase space. The basic counterexample to alternativity in this case is the function $\vec{x}^{2}:=$ $\sum_{\mu=1}^{d} x^{\mu} x^{\mu}$ for which

$$
\vec{x}^{2} \star\left(\vec{x}^{2} \star \vec{x}^{2}\right)=\left(\vec{x}^{2} \star \vec{x}^{2}\right) \star \vec{x}^{2}+3 \mathrm{i} \hbar \kappa^{2} R^{\mu \nu \rho} R^{\lambda \nu \rho} \delta_{\mu \sigma} x^{\sigma} p_{\lambda} .
$$

While the equation (5.14) cannot be solved in general by taking $\star$-inverses in $A_{\star}$, in the spirit of [7] we can regard the star-product operation $h \star g=\overline{\mathrm{f}}^{\alpha}(h) \cdot \overline{\mathrm{f}}_{\alpha}(g)$ as the action 
of the differential operator $h \star=\overline{\mathrm{f}}^{\alpha}(h) \overline{\mathrm{f}}_{\alpha}$ on the function $g$, and we can then consider the inverse of the differential operator $h \star$ with respect to the usual associative composition product of differential operators. We can actually refine this procedure by recalling the factorization $\mathcal{F}^{-1}=F^{-1} F_{R}^{-1}$ and the $*$-inverse expression (5.16) for the Moyal-Weyl twist $F$. We therefore write

$$
h \star g=\overline{\mathbf{f}}_{R}^{\alpha}(h) * \overline{\mathrm{f}}_{R \alpha}(g)
$$

and star-multiply with $*$ from the left by $h^{*-1}$. This gives

$$
h^{*-1} *(h \star g)=h^{*-1} * \overline{\mathrm{f}}_{R}^{\alpha}(h) * \overline{\mathrm{f}}_{R \alpha}(g),
$$

so that by defining the differential operator

$$
\mathrm{Y}_{h}:=\overline{\mathbf{f}}^{\beta}\left(h^{*-1} * \overline{\mathbf{f}}_{R}^{\alpha}(h)\right) \overline{\mathbf{f}}_{\beta} \overline{\mathbf{f}}_{R \alpha}
$$

we have

$$
\mathrm{Y}_{h}(g)=h^{*-1} * \overline{\mathbf{f}}_{R}^{\alpha}(h) * \overline{\mathbf{f}}_{R \alpha}(g)=h^{*-1} *(h \star g) .
$$

The differential operator $\mathrm{Y}_{h}$ can be regarded as a power series expansion in $\kappa$ (or equivalently in the $R$-flux) given by

$$
\begin{aligned}
\mathrm{Y}_{h} & =1+\sum_{n=1}^{\infty} \frac{1}{n !}\left(\frac{\mathrm{i} \kappa}{2}\right)^{n} \varepsilon^{A_{1} B_{1}} \cdots \varepsilon^{A_{n} B_{n}} \overline{\mathrm{f}}^{\beta}\left(h^{*-1} *\left(\boldsymbol{\partial}_{A_{1}} \cdots \boldsymbol{\partial}_{A_{n}} h\right)\right) \overline{\mathbf{f}}_{\beta} \boldsymbol{\partial}_{B_{1}} \cdots \boldsymbol{\partial}_{B_{n}} \\
& =: \sum_{n=0}^{\infty} \frac{(\mathrm{i} \kappa)^{n}}{n !} \mathrm{Y}_{h}^{(n)}
\end{aligned}
$$

with

$$
\left(\boldsymbol{\partial}_{A}\right)=\left(\begin{array}{c}
R^{\mu \nu \rho} p_{\nu} \partial_{\rho} \\
\partial_{\mu}
\end{array}\right) \quad \text { and } \quad\left(\varepsilon^{A B}\right)=\left(\begin{array}{cc}
0 & \mathbb{1}_{d} \\
-\mathbb{1}_{d} & 0
\end{array}\right) .
$$

The operator $\mathrm{Y}_{h}$ is invertible as a formal power series in $\kappa$ because it starts with the zeroth order term $\mathrm{Y}_{h}^{(0)}=1$; the first order term is

$$
\mathrm{Y}_{h}^{(1)}=\frac{1}{2} \overline{\mathrm{f}}^{\beta}\left(h^{*-1} *\left(R^{\mu \nu \rho} p_{\nu} \partial_{\rho} h\right)\right) \overline{\mathrm{f}}_{\beta} \partial_{\mu}-\frac{1}{2} \overline{\mathrm{f}}^{\beta}\left(h^{*-1} *\left(\partial_{\mu} h\right)\right) \overline{\mathrm{f}}_{\beta} R^{\mu \nu \rho} p_{\nu} \partial_{\rho} .
$$

We define

$$
\mathrm{X}_{h}=\sum_{n=0}^{\infty} \frac{(\mathrm{i} \kappa)^{n}}{n !} \mathrm{X}_{h}^{(n)}
$$

as the formal power series in $\kappa$ with coefficients in differential operators that satisfies (with composition $\circ$ of operators understood)

$$
\mathrm{X}_{h} \mathrm{Y}_{h}=\mathrm{id} .
$$

Then we can finally invert (5.14) to obtain the unique solution in terms of $h$ and $w$ :

$$
g=\left(\mathrm{X}_{h} \mathrm{Y}_{h}\right)(g)=\mathrm{X}_{h}\left(\mathrm{Y}_{h}(g)\right)=\mathrm{X}_{h}\left(h^{*-1} * w\right) .
$$


Crucially, this inversion cannot be written in general as $g=\tilde{h} \star w$ where $\tilde{h}$ is a function depending on $h$; the inversion formula (5.32) uses differential operators rather than the nonassociative algebra $A_{\star}$ of functions on its own.

To explicitly construct $\mathrm{X}_{h}$, we expand the left-hand side of (5.31) as a power series in $\kappa$ and equate it order by order with the right-hand side. The order zero term is $\mathrm{X}_{h}^{(0)}=1$, while the $n$-th order equation for $n \geq 1$ is

$$
\sum_{k=0}^{n}\left(\begin{array}{l}
n \\
k
\end{array}\right) \mathrm{X}_{h}^{(n-k)} \mathrm{Y}_{h}^{(k)}=0 .
$$

Since $\mathrm{Y}_{h}^{(0)}=1$, this yields a recursion relation for the differential operators $\mathrm{X}_{h}^{(n)}$ given by

$$
\mathrm{X}_{h}^{(n)}=-\sum_{k=1}^{n}\left(\begin{array}{l}
n \\
k
\end{array}\right) \mathrm{X}_{h}^{(n-k)} \mathrm{Y}_{h}^{(k)} .
$$

One easily shows by induction on $n$ that the solution to the recursion relation (5.34) with the initial condition $\mathrm{X}_{h}^{(0)}=1$ is

$$
\mathrm{X}_{h}^{(n)}=\sum_{|\vec{\lambda}|=n}(-1)^{l(\vec{\lambda})} \frac{n !}{\vec{\lambda} !} \mathrm{Y}_{h}^{(\vec{\lambda})},
$$

where the sum runs over all unordered sequences $\vec{\lambda}=\left(\lambda_{1}, \lambda_{2}, \ldots, \lambda_{l}\right)$ of positive integers $\lambda_{i}>0$ with length $l(\vec{\lambda})=l \leq n$ that partition $n$, i.e., $|\vec{\lambda}|:=\lambda_{1}+\lambda_{2}+\cdots+\lambda_{l}=n$, and we defined

$$
\mathrm{Y}_{h}^{(\vec{\lambda})}=\mathrm{Y}_{h}^{\left(\lambda_{1}\right)} \mathrm{Y}_{h}^{\left(\lambda_{2}\right)} \cdots \mathrm{Y}_{h}^{\left(\lambda_{l}\right)} \quad \text { and } \quad \vec{\lambda} !=\lambda_{1} ! \lambda_{2} ! \cdots \lambda_{l} !
$$

The first few orders are given by

$$
\begin{aligned}
& \mathrm{X}_{h}^{(0)}=1 \\
& \mathrm{X}_{h}^{(1)}=-\mathrm{Y}_{h}^{(1)} \\
& \mathrm{X}_{h}^{(2)}=-\mathrm{Y}_{h}^{(2)}+2 \mathrm{Y}_{h}^{(1)} \mathrm{Y}_{h}^{(1)} \\
& \mathrm{X}_{h}^{(3)}=-\mathrm{Y}_{h}^{(3)}+3 \mathrm{Y}_{h}^{(1)} \mathrm{Y}_{h}^{(2)}+3 \mathrm{Y}_{h}^{(2)} \mathrm{Y}_{h}^{(1)}-6 \mathrm{Y}_{h}^{(1)} \mathrm{Y}_{h}^{(1)} \mathrm{Y}_{h}^{(1)}
\end{aligned}
$$

Altogether, for the power series expansion (5.30) we obtain

$$
\mathrm{X}_{h}=1+\sum_{\vec{\lambda}} \frac{(\mathrm{i} \kappa)^{|\vec{\lambda}|}}{\vec{\lambda} !}(-1)^{l(\vec{\lambda})} \mathrm{Y}_{h}^{(\vec{\lambda})}
$$

where here the sum runs through all unordered finite sequences $\vec{\lambda}$ of positive integers.

Let us look at an explicit example. For the tachyon vertex operators $h(x)=\mathrm{e}^{\mathrm{i} k_{\mu} x^{\mu}}$, using antisymmetry of $R^{\mu \nu \rho}$ we easily obtain from (5.27) the differential operator

$$
\mathrm{Y}_{\mathrm{e}^{\mathrm{i} k_{\mu} x^{\mu}}}=\exp \left(-\kappa R^{\mu \nu \rho} k_{\mu} p_{\nu} \partial_{\rho}\right)
$$


with inverse

$$
\mathrm{X}_{\mathrm{e}^{\mathrm{i} k \mu x^{\mu}}}=\exp \left(\kappa R^{\mu \nu \rho} k_{\mu} p_{\nu} \partial_{\rho}\right) .
$$

Then the equation $\mathrm{e}^{\mathrm{i} k_{\mu} x^{\mu}} \star g=w$ has the unique solution

$$
g(x, p)=\left(\mathrm{e}^{-\mathrm{i} k_{\mu} x^{\mu}} * w\right)(x+\kappa R(k, p), p)=\mathrm{e}^{-\mathrm{i} k_{\mu} x^{\mu}} w\left(x+\kappa R(k, p), p-\frac{\hbar}{2} k\right)
$$

where $R(k, p)^{\mu}:=R^{\mu \nu \rho} k_{\nu} p_{\rho}$. When restricted to the zero momentum leaf of phase space, this yields $g(x, 0)=\mathrm{e}^{-\mathrm{i} k_{\mu} x^{\mu}} w\left(x,-\frac{\hbar}{2} k\right)$, which for functions $w(x)$ depending only on spacetime coordinates further reduces to $g(x, 0)=\mathrm{e}^{-\mathrm{i} k_{\mu} x^{\mu}} w(x)$.

\subsection{Levi-Civita connection}

It is straightforward to extend the analysis of section 5.2 to construct a nonassociative version of the Levi-Civita connection in Riemannian geometry. If the differential operator $\mathrm{Y}_{h}$ is also matrix-valued, then we just have to interpret products as the composition of operators together with matrix multiplication; the algebraic manipulations of section 5.2 are identical because $h, g$ and $w$ were treated there as abstract symbols and not as commuting functions. Hence all formulas are also valid if $h, g$ and $w$ in (5.14) are matrix-valued functions and matrix multiplication is understood in star-products. Therefore we will only sketch the main steps.

Let $\mathrm{G}^{-1}=\left(\mathrm{G}^{M N}\right)$ be the inverse matrix of the matrix $\mathrm{G}=\left(\mathrm{G}_{M N}\right)$ describing the string effective metric: $\mathrm{G}^{M C} \cdot \mathrm{G}_{C N}=\mathrm{G}_{N C} \cdot \mathrm{G}^{C M}=\delta_{N}^{M}$. There is the closed expression

$$
\mathrm{G}^{-1}=\left(\mathbb{1}_{2 d}+\mathrm{i} \kappa \mathrm{g}^{-1} \mathscr{R} \partial \mathrm{g}\right)^{-1} \mathrm{~g}^{-1}
$$

where $\mathscr{R} \partial \mathrm{g}=\left(\mathscr{R}^{E F}{ }_{M} \partial_{E} \mathrm{~g}_{N F}\right)$ while $\mathrm{g}^{-1}=\left(\mathrm{g}^{M C}\right)$ is the inverse of $\mathrm{g}=\left(\mathrm{g}_{C N}\right)$ :

$$
\mathrm{g}^{M C} \cdot \mathrm{g}_{C N}=\mathrm{g}_{N C} \cdot \mathrm{g}^{C M}=\delta_{N}^{M},
$$

and $\left(\mathbb{1}_{2 d}+\mathrm{i} \kappa \mathrm{g}^{-1} \mathscr{R} \partial \mathrm{g}\right)^{-1}$ is understood as a geometric series, so that

$$
\mathrm{G}^{M C}=\mathrm{g}^{M C}-\mathrm{i} \kappa \mathrm{g}^{M N} \mathscr{R}^{A B}{ }_{N}\left(\partial_{A} \mathrm{~g}_{B E}\right) \mathrm{g}^{E C}+O\left(\kappa^{2}\right) .
$$

Let $\mathrm{G}^{*-1}=\left(\mathrm{G}^{* M N}\right)$ be the $*$-inverse of the matrix $\mathrm{G}=\left(\mathrm{G}_{M N}\right)$ :

$$
\mathrm{G}^{* M C} * \mathrm{G}_{C N}=\mathrm{G}_{N C} * \mathrm{G}^{* C M}=\delta_{N}^{M} .
$$

It can be expressed explicitly as a power series in $\hbar$ given by [7]

$$
\mathrm{G}^{* M N}=\mathrm{G}^{M N}+\sum_{n=1}^{\infty} \mathrm{G}^{M C} *\left(\mathbb{1}_{2 d}-\mathrm{G} * \mathrm{G}^{-1}\right)_{C}^{* n}
$$

Let $\mathrm{Y}_{\mathrm{G}}$ be the matrix-valued differential operator defined by

$$
\mathrm{Y}_{\mathrm{G}}:=\left(\mathrm{Y}_{\mathrm{G}} \stackrel{M}{N}\right), \quad \mathrm{Y}_{\mathrm{G}}{ }_{N}^{M}:=\overline{\mathrm{f}}^{\beta}\left(\mathrm{G}^{* M C} * \overline{\mathrm{f}}_{R}^{\alpha}\left(\mathrm{G}_{C N}\right)\right) \overline{\mathrm{f}}_{\beta} \overline{\mathrm{f}}_{R \alpha}=: \sum_{n=0}^{\infty} \frac{(\mathrm{i} \kappa)^{n}}{n !} \mathrm{Y}_{\mathrm{G}}^{(n)} \stackrel{M}{N}
$$


so that acting on a function $g$ gives $\mathrm{Y}_{\mathrm{G}}{ }_{N}^{M}(g)=\mathrm{G}^{* M C} * \overline{\mathrm{f}}_{R}^{\alpha}\left(\mathrm{G}_{C N}\right) * \overline{\mathrm{f}}_{R \alpha}(g)$. The zeroth and first order terms are given by

$$
\begin{aligned}
\mathrm{Y}_{\mathrm{G} N}^{(0) M} & =\delta_{N}^{M} \\
\mathrm{Y}_{\mathrm{G} N}^{(1) M} & =\frac{1}{2} \overline{\mathrm{f}}^{\beta}\left(\mathrm{G}^{* M C} *\left(R^{\mu \nu \rho} p_{\nu} \partial_{\rho} \mathrm{G}_{C N}\right)\right) \overline{\mathrm{f}}_{\beta} \partial_{\mu}-\frac{1}{2} \overline{\mathrm{f}}^{\beta}\left(\mathrm{G}^{* M C} *\left(\partial_{\mu} \mathrm{G}_{C N}\right)\right) \overline{\mathrm{f}}_{\beta} R^{\mu \nu \rho} p_{\nu} \partial_{\rho} \\
& =\frac{1}{2} \varepsilon^{A B} \overline{\mathrm{f}}^{\beta}\left(\mathrm{G}^{* S C(0)} * \boldsymbol{\partial}_{A} \mathrm{~g}_{C M}\right) \overline{\mathrm{f}}_{\beta} \partial_{B}
\end{aligned}
$$

where in the last equality we used the notation (5.28).

Following the formalism of section 5.2, we determine from (5.13) the connection coefficients

$$
\Gamma_{A D}^{S}=\mathrm{G}^{* S C} * \mathrm{~W}_{C A D}+\sum_{\vec{\lambda}} \frac{(\mathrm{i} \kappa)^{|\vec{\lambda}|}}{\vec{\lambda} !}(-1)^{l(\vec{\lambda})} \mathrm{Y}_{\mathrm{G}}^{(\vec{\lambda}) S}{ }_{M}\left(\mathrm{G}^{* M C} * \mathrm{~W}_{C A D}\right)
$$

where

$$
\mathrm{W}_{C A D}=\frac{1}{2}\left(\partial_{D} \mathrm{~g}_{A C}+\partial_{A} \mathrm{~g}_{D C}-\partial_{C} \mathrm{~g}_{A D}+\mathrm{i} \kappa \mathscr{R}^{E F}{ }_{C}\left(\partial_{E} \partial_{D} \mathrm{~g}_{A F}+\partial_{E} \partial_{A} \mathrm{~g}_{D F}\right)\right)
$$

and

$$
\mathrm{Y}_{\mathrm{G}}^{(\vec{\lambda}) S}{ }_{M}^{S}=\mathrm{Y}_{\mathrm{G}}^{\left(\lambda_{1}\right) N_{M}}{ }_{M} \circ \mathrm{Y}_{\mathrm{G}}^{\left(\lambda_{2}\right) N_{2}}{ }_{N_{1}} \circ \cdots \circ \mathrm{Y}_{\mathrm{G}}^{\left(\lambda_{l}\right) S} N_{l-1}
$$

for $\vec{\lambda}=\left(\lambda_{1}, \lambda_{2}, \ldots, \lambda_{l}\right)$. We also recall from section 5.2 the notation $\vec{\lambda} !=\lambda_{1} ! \lambda_{2} ! \cdots \lambda_{l}$ ! and $|\vec{\lambda}|=\lambda_{1}+\lambda_{2}+\cdots+\lambda_{l}$, and that the sum in (5.49) runs over all finite sequences $\vec{\lambda}$ of positive integers. In particular, for $|\vec{\lambda}|=1$ the only contribution to the sum is given by the term $\mathrm{Y}_{\mathrm{G} N}^{(1)}$ in $(5.48)$.

In order to understand better the expansion (5.49) of the Levi-Civita connection, we will now extract the leading non-trivial terms. For this, we have to expand every tensor entering into this expression up to first order in $\kappa$ and first order in $\hbar$; we expect non-trivial nonassociativity contributions in the $O(\kappa \hbar)=O\left(\ell_{s}^{3}\right)$ terms. For any tensor $T$ we write

$$
T=\sum_{n=0}^{\infty} T^{(n)} \quad \text { with } \quad T^{(n)}=\sum_{m=0}^{\infty} T^{(n, m)}
$$

where by $T^{(n)}$ we understand the term in the power series expansion of $T$ which is of $n$-th order in $\kappa$, and $T^{(n, m)}$ is the term in the double power series expansion of $T$ which is $n$-th order in $\kappa$ and $m$-th order in $\hbar$. We write (5.50) as

$$
\begin{aligned}
\mathrm{W}_{C A D} & =\mathrm{g}_{C M} \Gamma_{A D}^{\mathrm{LC} M}+\mathrm{i} \kappa \mathscr{R}^{E F}{ }_{C} \partial_{E}\left(\mathrm{~g}_{F M} \Gamma_{A D}^{\mathrm{LC} M}\right) \\
& =: \mathrm{W}_{C A D}^{(0)}+\mathrm{W}_{C A D}^{(1)},
\end{aligned}
$$

where

$$
\Gamma_{A D}^{\mathrm{LC} M}=\frac{1}{2} \mathrm{~g}^{M Q}\left(\partial_{D} \mathrm{~g}_{A Q}+\partial_{A} \mathrm{~g}_{D Q}-\partial_{Q} \mathrm{~g}_{A D}\right)
$$

is the usual classical Levi-Civita connection, which is zeroth order in $\kappa$ and zeroth order in $\hbar$ if the metric $\mathrm{g}_{M N}$ is independent of $\kappa$ and $\hbar$. Then the definition in (5.53) is exact in $\kappa$, 
i.e., there are no higher order terms $\left(\mathrm{W}_{C A D}^{(n)}=0\right.$ for $\left.n \geq 2\right)$. The effective metric has the expansion

$$
\begin{aligned}
\mathrm{G}_{M N} & =\mathrm{g}_{M N}+\mathrm{i} \kappa \mathscr{R}^{E F}{ }_{M} \partial_{E} \mathrm{~g}_{N F} \\
& =: \mathrm{G}_{M N}^{(0)}+\mathrm{G}_{M N}^{(1)}, \\
\mathrm{G}^{S C} & =\mathrm{g}^{S C}-\mathrm{i} \kappa \mathrm{g}^{S M} \mathscr{R}^{E F}{ }_{M}\left(\partial_{E} \mathrm{~g}_{N F}\right) \mathrm{g}^{N C}+O\left(\kappa^{2}\right) \\
& =: \mathrm{G}^{S C(0)}+\mathrm{G}^{S C(1)}+O\left(\kappa^{2}\right) .
\end{aligned}
$$

Recalling (5.48) we can now write the expansion of $\Gamma_{A D}^{S}$ in (5.49) up to first order in $\kappa$ and first order in $\hbar$ as

$$
\begin{aligned}
\Gamma_{A D}^{S(0)}= & \mathrm{G}^{* S C(0)} * \mathrm{~W}_{C A D}^{(0)} \\
=: & \Gamma_{A D}^{S(0,0)}+\Gamma_{A D}^{S(0,1)}+O\left(\hbar^{2}\right), \\
\Gamma_{A D}^{S(1)}= & \mathrm{G}^{* S C(0)} * \mathrm{~W}_{C A D}^{(1)}+\mathrm{G}^{* S C(1)} * \mathrm{~W}_{C A D}^{(0)} \\
& -\frac{\mathrm{i} \kappa}{2} \varepsilon^{K L} \mathrm{G}^{* S Q(0)} * \partial_{K} \mathrm{~g}_{Q M} * \partial_{L}\left(\mathrm{G}^{* M C(0)} * \mathrm{~W}_{C A D}^{(0)}\right) \\
=: & \Gamma_{A D}^{S(1,0)}+\Gamma_{A D}^{S(1,1)}+O\left(\hbar^{2}\right) .
\end{aligned}
$$

To explicitly calculate these terms we observe from (5.46) that

$$
\mathrm{G}^{* S C}=2 \mathrm{G}^{S C}-\mathrm{G}^{S P} * \mathrm{G}_{P Q} * \mathrm{G}^{Q C}+O\left(\hbar^{2}\right),
$$

so that

$$
\begin{aligned}
\mathrm{G}^{* S C(0)}= & 2 \mathrm{~g}^{S C}-\mathrm{g}^{S P} * \mathrm{~g}_{P Q} * \mathrm{~g}^{Q C}+O\left(\hbar^{2}\right) \\
= & \mathrm{g}^{S C}-\frac{\mathrm{i} \hbar}{2}\left(\partial_{\mu} \mathrm{g}^{S P} \tilde{\partial}^{\mu} \mathrm{g}_{P Q}-\tilde{\partial}^{\mu} \mathrm{g}^{S P} \partial_{\mu} \mathrm{g}_{P Q}\right) \mathrm{g}^{Q C}+O\left(\hbar^{2}\right) \\
= & \mathrm{G}^{* S C(0,0)}+\mathrm{G}^{* S C(0,1)}+O\left(\hbar^{2}\right), \\
\mathrm{G}^{* S C(1)}= & 2 \mathrm{G}^{S C(1)}-\mathrm{g}^{S P} * \mathrm{~g}_{P Q} * \mathrm{G}^{Q C(1)}-\mathrm{g}^{S P} * \mathrm{G}_{P Q}^{(1)} * \mathrm{~g}^{Q C}-\mathrm{G}^{S P(1)} * \mathrm{~g}_{P Q} * \mathrm{~g}^{Q C}+O\left(\hbar^{2}\right) \\
= & -\mathrm{i} \kappa \mathscr{R}^{E F}{ }_{M} \mathrm{~g}^{S M}\left(\partial_{E} \mathrm{~g}_{N F}\right) \mathrm{g}^{N C} \\
& -\frac{\hbar \kappa}{2} \mathscr{R}^{E F}{ }_{M}\left(\partial _ { E } \mathrm { g } _ { N F } \left(\mathrm{~g}^{Q M} \mathrm{~g}^{N C}\left(\partial_{\mu} \mathrm{g}^{S P} \tilde{\partial}^{\mu} \mathrm{g}_{P Q}-\tilde{\partial}^{\mu} \mathrm{g}^{S P} \partial_{\mu} \mathrm{g}_{P Q}\right)\right.\right. \\
& \left.+\mathrm{g}^{S M} \mathrm{~g}^{Q C}\left(\partial_{\mu} \mathrm{g}^{N P} \tilde{\partial}^{\mu} \mathrm{g}_{P Q}-\tilde{\partial}^{\mu} \mathrm{g}^{N P} \partial_{\mu} \mathrm{g}_{P Q}\right)+\partial_{\mu} \mathrm{g}^{S M} \tilde{\partial}^{\mu} \mathrm{g}^{N C}-\tilde{\partial}^{\mu} \mathrm{g}^{S M} \partial_{\mu} \mathrm{g}^{N C}\right) \\
& -\left(\partial_{\mu} \partial_{E} \mathrm{~g}_{N F}\right)\left(\mathrm{g}^{S M} \tilde{\partial}^{\mu} \mathrm{g}^{N C}-\mathrm{g}^{N C} \tilde{\partial}^{\mu} \mathrm{g}^{S M}\right) \\
& \left.+\left(\tilde{\partial}^{\mu} \partial_{E} \mathrm{~g}_{N F}\right)\left(\mathrm{g}{ }^{S M} \partial_{\mu} \mathrm{g}^{N C}-\mathrm{g}^{N C} \partial_{\mu} \mathrm{g}^{S M}\right)\right)+O\left(\hbar^{2}\right) \\
=: & \mathrm{G}^{* S C(1,0)}+\mathrm{G}^{* S C(1,1)}+O\left(\hbar^{2}\right) .
\end{aligned}
$$

We can now compute the first non-trivial terms of the Levi-Civita connection as defined 
in (5.57) and (5.58). We obtain

$$
\begin{aligned}
& \Gamma_{A D}^{S(0,0)}=\Gamma_{A D}^{\mathrm{LC} S}, \\
& \Gamma_{A D}^{S(0,1)}=\frac{\mathrm{i} \hbar}{2}\left(-\left(\partial_{\mu} \mathrm{g}^{S P} \tilde{\partial}^{\mu} \mathrm{g}_{P Q}-\tilde{\partial}^{\mu} \mathrm{g} P P \partial_{\mu} \mathrm{g}_{P Q}\right) \Gamma_{A D}^{\mathrm{LC} Q}\right. \\
& \left.+\partial_{\mu} \mathrm{g}^{S C} \tilde{\partial}^{\mu}\left(\mathrm{g}_{C M} \Gamma_{A D}^{\mathrm{LC} M}\right)-\tilde{\partial}^{\mu} \mathrm{g} C \partial_{\mu}\left(\mathrm{g}_{C M} \Gamma_{A D}^{\mathrm{LC} M}\right)\right) \\
& =-\frac{\mathrm{i} \hbar}{2} \mathrm{~g}^{S P}\left(\left(\partial_{\mu} \mathrm{g}_{P Q}\right) \tilde{\partial}^{\mu} \Gamma_{A D}^{\mathrm{LC} Q}-\left(\tilde{\partial}^{\mu} \mathrm{g}_{P Q}\right) \partial_{\mu} \Gamma_{A D}^{\mathrm{LC} Q}\right), \\
& \Gamma_{A D}^{S(1,0)}=\mathrm{i} \kappa R^{\alpha \beta \gamma}\left(\tilde{\mathrm{g}}_{\gamma}^{S} \mathrm{~g}_{\beta N}\left(\partial_{\alpha} \Gamma_{A D}^{\mathrm{LC} N}\right)-\mathrm{g}^{S M} p_{\beta}\left(\partial_{\gamma} \mathrm{g}_{M N}\right) \partial_{\alpha} \Gamma_{A D}^{\mathrm{LC} N}\right), \\
& \Gamma_{A D}^{S(1,1)}=\frac{\hbar \kappa}{2} R^{\alpha \beta \gamma}\left[-\partial_{\mu} \tilde{\mathrm{g}}_{\gamma}^{S} \tilde{\partial}^{\mu} \partial_{\alpha}\left(\mathrm{g}_{\beta N} \Gamma_{A D}^{\mathrm{LC} N}\right)+\tilde{\partial}^{\mu} \tilde{\mathrm{g}}_{\gamma}^{S} \partial_{\mu} \partial_{\alpha}\left(\mathrm{g}_{\beta N} \Gamma_{A D}^{\mathrm{LC} N}\right)\right. \\
& +\left(\partial_{\mu} \mathrm{g}^{S P} \tilde{\partial}^{\mu} \mathrm{g}_{P Q}-\tilde{\partial}^{\mu} \mathrm{g}^{S P} \partial_{\mu} \mathrm{g}_{P Q}\right) \tilde{\mathrm{g}}_{\gamma}^{Q} \partial_{\alpha}\left(\mathrm{g}_{\beta N} \Gamma_{A D}^{\mathrm{LC} N}\right) \\
& +\partial_{\mu}\left(\tilde{\mathrm{g}}_{\gamma}^{S} \mathrm{~g}^{N C} \partial_{\alpha} \mathrm{g}_{N \beta}\right) \tilde{\partial}^{\mu}\left(\mathrm{g}_{C T} \Gamma_{A D}^{\mathrm{LC} T}\right)-\tilde{\partial}^{\mu}\left(\tilde{\mathrm{g}}_{\gamma}^{S} \mathrm{~g}^{N C} \partial_{\alpha} \mathrm{g}_{N \beta}\right) \partial_{\mu}\left(\mathrm{g}_{C T} \Gamma_{A D}^{\mathrm{LC} T}\right) \\
& -\partial_{\alpha} \mathrm{g}_{N \beta}\left(\tilde{\mathrm{g}}_{\gamma}^{S}\left(\partial_{\mu} \mathrm{g}^{S P} \tilde{\partial}^{\mu} \mathrm{g}_{P Q}-\tilde{\partial}^{\mu} \mathrm{g}^{S P} \partial_{\mu} \mathrm{g}_{P Q}\right) \Gamma_{A D}^{\mathrm{LC} N}\right. \\
& +\tilde{\mathrm{g}}_{\gamma}^{S}\left(\partial_{\mu} \mathrm{g}^{N P} \tilde{\partial}^{\mu} \mathrm{g}_{P Q}-\tilde{\partial}^{\mu} \mathrm{g}^{N P} \partial_{\mu} \mathrm{g}_{P Q}\right) \Gamma_{A D}^{\mathrm{LC} Q} \\
& \left.+\left(\partial_{\mu} \tilde{\mathrm{g}}_{\gamma}^{S} \tilde{\partial}^{\mu} \mathrm{g}^{N C}-\tilde{\partial}^{\mu} \tilde{\mathrm{g}}_{\gamma}^{S} \partial_{\mu} \mathrm{g}^{N C}\right) \mathrm{g}_{C T} \Gamma_{A D}^{\mathrm{LC} T}\right) \\
& +\left(\partial_{\mu} \partial_{\alpha} \mathrm{g}_{N \beta}\right)\left(\tilde{\mathrm{g}}_{\gamma}^{S}\left(\tilde{\partial}^{\mu} \mathrm{g}^{N C}\right) \mathrm{g}_{C T} \Gamma_{A D}^{\mathrm{LC} T}-\left(\tilde{\partial}^{\mu} \tilde{\mathrm{g}}_{\gamma}^{S}\right) \Gamma_{A D}^{\mathrm{LC} N}\right) \\
& -\left(\tilde{\partial}^{\mu} \partial_{\alpha} \mathrm{g}_{N \beta}\right)\left(\tilde{\mathrm{g}}_{\gamma}^{S}\left(\partial_{\mu} \mathrm{g}^{N C}\right) \mathrm{g}_{C T} \Gamma_{A D}^{\mathrm{LC} T}-\left(\partial_{\mu} \tilde{\mathrm{g}}_{\gamma}^{S}\right) \Gamma_{A D}^{\mathrm{LC} N}\right) \\
& +p_{\beta}\left(\partial_{\mu}\left(\mathrm{g}^{S Q} \partial_{\gamma} \mathrm{g}_{Q P}\right) \tilde{\partial}^{\mu} \partial_{\alpha} \Gamma_{A D}^{\mathrm{LC} P}-\tilde{\partial}^{\mu}\left(\mathrm{g}^{S Q} \partial_{\gamma} \mathrm{g}_{Q P}\right) \partial_{\mu} \partial_{\alpha} \Gamma_{A D}^{\mathrm{LC} P}\right) \\
& +p_{\beta} \mathrm{g}^{S Q}\left(\partial_{\gamma} \mathrm{g}_{Q P}\right) \partial_{\alpha}\left(\partial_{\mu} \mathrm{g}^{P C} \tilde{\partial}^{\mu}\left(\mathrm{g}_{C T} \Gamma_{A D}^{\mathrm{LC} T}\right)-\tilde{\partial}^{\mu} \mathrm{g}^{P C} \partial_{\mu}\left(\mathrm{g}_{C T} \Gamma_{A D}^{\mathrm{LC} T}\right)\right. \\
& \left.-\left(\partial_{\mu} \mathrm{g}^{P X} \tilde{\partial}^{\mu} \mathrm{g}_{X Y}-\tilde{\partial}^{\mu} \mathrm{g}^{P X} \partial_{\mu} \mathrm{g} X Y\right) \Gamma_{A D}^{\mathrm{LC} Y}\right) \\
& +p_{\beta}\left(\partial_{\mu} \mathrm{g}^{S Q} \tilde{\partial}^{\mu} \partial_{\gamma} \mathrm{g}_{Q P}-\tilde{\partial}^{\mu} \mathrm{g} S Q \partial_{\mu} \partial_{\gamma} \mathrm{g}_{Q P}\right) \partial_{\alpha} \Gamma_{A D}^{\mathrm{LC} P} \\
& -p_{\beta}\left(\partial_{\mu} \mathrm{g}^{S M} \tilde{\partial}^{\mu} \mathrm{g}_{M N}-\tilde{\partial}^{\mu} \mathrm{g}^{S M} \partial_{\mu} \mathrm{g}_{M N}\right) \mathrm{g}^{N Q}\left(\partial_{\gamma} \mathrm{g}_{Q P}\right) \partial_{\alpha} \Gamma_{A D}^{\mathrm{LC} P} \\
& \left.+\left(\partial_{\alpha} \mathrm{g}^{S Q}\right)\left(\partial_{\beta} \mathrm{g}_{Q P}\right) \partial_{\gamma} \Gamma_{A D}^{\mathrm{LC} P}\right] \text {, }
\end{aligned}
$$

where $\tilde{\mathrm{g}}_{\gamma}^{S}=\mathrm{g}^{S M} \delta_{M, \tilde{x}_{\gamma}}$ is the part of the inverse metric tensor $\mathrm{g}^{M N}$ with at least one index in momentum space.

We offer the following remarks on the expanded Levi-Civita connection:

1. Terms that are of type $(0,1)$ and $(1,0)$, i.e., proportional to $\hbar$ or to $\kappa$ alone, are imaginary; this is analogous to what happens in gravity theories on Moyal-Weyl spaces [7]. On the other hand, the term of type $(1,1)$, i.e., proportional to $\hbar \kappa=\frac{\ell_{s}^{3}}{6}$, is real; it represents the non-trivial nonassociativity contribution.

2. If we restrict ourselves to a metric that does not depend on the momenta $p_{\mu}$, then (5.63) vanishes and all terms but $\frac{\ell_{s}^{3}}{6} R^{\alpha \beta \gamma}\left(\partial_{\alpha} \mathrm{g}^{S Q}\right)\left(\partial_{\beta} \mathrm{g}_{Q P}\right) \partial_{\gamma} \Gamma_{A D}^{\mathrm{LC} P}$ in (5.65) vanish. This remaining term is just the associator acting on a product of classical metric tensors and the classical Levi-Civita connection (5.54), as is anticipated from the way in which we extracted the connection coefficients $\Gamma_{A D}^{S}$ from (5.13). 
3. If we restrict to a metric with no indices in momentum space, i.e., $\tilde{\mathrm{g}}_{\gamma}^{S}=0$, then many terms in (5.65) vanish. The terms that remain are those linear in momenta $p_{\beta}$ and the associator term $\frac{\ell_{s}^{3}}{6} R^{\alpha \beta \gamma}\left(\partial_{\alpha} \mathrm{g} S Q\right)\left(\partial_{\beta} \mathrm{g}_{Q P}\right) \partial_{\gamma} \Gamma_{A D}^{\mathrm{LC} P}$. If we further restrict to a momentum-independent metric and constrain it to the zero momentum leaf in phase space, we obtain a real-valued Levi-Civita connection on spacetime which is independent of $\hbar$ and with a non-trivial $R$-flux dependence due to nonassociativity. However, we must keep the momentum arbitrary for the time being as such terms will make non-trivial contributions to the Ricci tensor below.

\subsection{Einstein equations}

Given an arbitrary metric tensor $\mathrm{g}$ on phase space $\mathcal{M}$ with nonassociative deformation induced by a constant $R$-flux, we have constructed its unique Levi-Civita connection in section 5.3. Recalling the definition of the Ricci tensor from section 4.6, we can therefore consider the vacuum Einstein equations on this nonassociative deformation of $\mathcal{M}$. They read $\operatorname{Ric}^{\star}=0$, or in components as

$$
\operatorname{Ric}_{B C}=0
$$

This equation is a deformation in $\kappa$ and $\hbar$ of the usual vacuum Einstein equations for gravity. It is easy to see that the flat space metric $\mathrm{g}_{A B}=\eta_{A B}$ gives a vanishing Levi-Civita connection and hence solves the vacuum equations (5.66). Indeed, in this case $\mathrm{G}_{A B}=\eta_{A B}$ and all star-products reduce to the usual pointwise products, because there is no dependence on the phase space coordinates $x$ and $p$ at all.

A more general solution can be easily obtained by considering metrics $g_{A B}(p)$ that depend only on the momentum coordinates. For these metrics we have $\mathrm{G}_{A B}=\mathrm{g}_{A B}$ and the usual inverse $\mathrm{G}^{A B}=\mathrm{g}^{A B}$ is also the $\star$-inverse. Indeed here too all star-products drop out because the twist $F_{R}$ always involves vector fields $\partial_{\mu}$ and so acts trivially. Moreover, the Moyal-Weyl twist $F$ also acts trivially on functions that depend only on the momentum coordinates: each summand in (2.4) contains always at least one vector field $\partial_{\mu}$ that acts trivially in this case. This implies that if a metric $\mathrm{g}_{A B}(p)$ solves the vacuum Einstein equations in the classical case, then it remains a solution of the vacuum Einstein equations also when the $R$-flux is turned on and hence it is also a solution of (5.66). See [3] for further details in the noncommutative case.

\subsection{Spacetime field equations}

Recall that our original motivation was to obtain a nonassociative theory of gravity on spacetime. The correct way in which to obtain a reduction to spacetime dynamics from the nonassociative phase space formalism was explained in [5]: we start from tensors on $M=\mathbb{R}^{d}$, lift them to tensors on $\mathcal{M}=T^{*} M=\mathbb{R}^{d} \times\left(\mathbb{R}^{d}\right)^{*}$, construct new composite tensors using the nonassociative deformation of the geometry of $\mathcal{M}$, reorder the result using the associator, and then project back to $M$. The lift from $M$ to $\mathcal{M}$ for functions and more generally for forms is just the pullback of forms using the canonical projection $\pi: \mathcal{M}=$ $T^{*} M \rightarrow M$. In the opposite direction, using the embedding $\sigma: M \rightarrow \mathcal{M}=\mathbb{R}^{d} \times\left(\mathbb{R}^{d}\right)^{*}$ 
given by the zero section $x \mapsto \sigma(x)=(x, 0)$, we pull back forms on $\mathcal{M}$ to forms on $M$. For example, the $n$-product of functions on $M$ defined in ([5], eq. (3.7)) immediately extends to the $n$-exterior product of forms on $M$ as

$$
\wedge_{\star}^{(n)}\left(\omega_{1}, \omega_{2}, \ldots, \omega_{n}\right):=\sigma^{*}\left[\left(\cdots\left(\left(\pi^{*} \omega_{1} \wedge_{\star} \pi^{*} \omega_{2}\right) \wedge_{\star} \pi^{*} \omega_{3}\right) \wedge_{\star} \cdots\right) \wedge_{\star} \pi^{*} \omega_{n}\right] .
$$

The lifts of vector fields are obtained by considering a foliation of $\mathcal{M}$ via constant momentum leaves, with each leaf being diffeomorphic to $M$. Explicitly, the coordinate basis vector field $\partial_{\mu}$ on $M$ lifts to the coordinate basis vector field $\partial_{\mu}$ on $\mathcal{M}$, and more generally $v^{\mu}(x) \partial_{\mu} \mapsto \pi^{*}\left(v^{\mu}\right)(x, p) \partial_{\mu}$, where $\pi^{*}\left(v^{\mu}\right)(x, p)=\left(v^{\mu}\right)(\pi(x, p))=v^{\mu}(x)$. In the opposite direction, vector fields on $\mathcal{M}$ are projected to vector fields on $M$ via the zero section $\sigma: M \rightarrow \mathcal{M}$ as $v^{\mu}(x, p) \partial_{\mu}+\tilde{v}_{\mu}(x, p) \tilde{\partial}^{\mu} \mapsto v^{\mu}(x, 0) \partial_{\mu}$.

The lift of a metric tensor on $M$ to a metric tensor on $\mathcal{M}$ requires an additional structure: a nondegenerate bilinear form on the cotangent bundle $\mathcal{M}=T^{*} M$, i.e., a bilinear form on each cotangent space $T_{x}^{*} M$, which we denote by $\mathrm{h}(x)^{\mu \nu} \mathrm{d} \tilde{x}_{\mu} \otimes \mathrm{d} \tilde{x}_{\nu}$. Then a metric $\mathrm{g}_{\mu \nu}(x) \mathrm{d} x^{\mu} \otimes \mathrm{d} x^{\nu}$ on $M$ is lifted to the metric $\hat{\mathrm{g}}_{M N} \mathrm{~d} x^{M} \otimes \mathrm{d} x^{N}$ on $\mathcal{M}$ given by

$$
\left(\hat{\mathrm{g}}_{M N}(x)\right)=\left(\begin{array}{cc}
\mathrm{g}_{\mu \nu}(x) & 0 \\
0 & \mathrm{~h}^{\mu \nu}(x)
\end{array}\right) .
$$

Next we rewrite $\hat{\mathrm{g}}_{M N} \mathrm{~d} x^{M} \otimes \mathrm{d} x^{N}$ in terms of the star-tensor product as

$$
\hat{\mathrm{g}}_{M N} \mathrm{~d} x^{M} \otimes \mathrm{d} x^{N}=\mathrm{g}_{M N} \star\left(\mathrm{d} x^{M} \otimes_{\star} \mathrm{d} x^{N}\right) .
$$

We thus obtain metric coefficients that have a linear correction in the $R$-flux given by

$$
\left(\mathrm{g}_{M N}(x)\right)=\left(\begin{array}{cc}
\mathrm{g}_{\mu \nu}(x) & \frac{\mathrm{i} \kappa}{2} R^{\sigma \nu \alpha} \partial_{\sigma} \mathrm{g}_{\mu \alpha} \\
\frac{\mathrm{i} \kappa}{2} R^{\sigma \mu \alpha} \partial_{\sigma} \mathrm{g}_{\alpha \nu} & \mathrm{h}^{\mu \nu}(x)
\end{array}\right) .
$$

Then the Ricci tensor on spacetime is the pullback

$$
\operatorname{Ric}^{\star \circ}:=\sigma^{*}\left(\operatorname{Ric}^{\star}\right)
$$

Recalling the expansion (4.81) of the Ricci tensor in the good basis, we obtain

$$
\operatorname{Ric}^{\star \circ}=\operatorname{Ric}_{\mu \nu}^{\circ} \mathrm{d} x^{\mu} \otimes \mathrm{d} x^{\nu},
$$

where the products are the usual undeformed products because the 3 -tensor product in a good basis is the usual tensor product:

$$
\otimes_{\star}^{(3)}\left(f, \mathrm{~d} x^{\mu}, \mathrm{d} x^{\nu}\right):=\sigma^{*}\left(\left(\pi^{*} f \otimes_{\star} \pi^{*} \mathrm{~d} x^{\mu}\right) \otimes_{\star} \pi^{*} \mathrm{~d} x^{\nu}\right)=f \mathrm{~d} x^{\mu} \otimes \mathrm{d} x^{\nu} .
$$

Comparing (5.71) with (4.81) and (5.72) leads to the simple result that the nonassociative spacetime Ricci tensor is obtained form the phase space Ricci tensor simply by restricting the components $\operatorname{Ric}_{M N}$ to spacetime directions and setting the momentum dependence to zero:

$$
\operatorname{Ric}_{\mu \nu}^{\circ}(x)=\sigma^{*}\left(\operatorname{Ric}_{\mu \nu}\right)(x, p)=\operatorname{Ric}_{\mu \nu}(x, 0) .
$$


The spacetime vacuum equations for nonassociative gravity then read as

$$
\operatorname{Ric}_{\mu \nu}^{\circ}=0 \text {. }
$$

We observe that the flat metric $\mathrm{g}_{\mu \nu}(x)=\eta_{\mu \nu}, \mathrm{h}^{\mu \nu}(x)=\eta^{\mu \nu}$ is a solution of (5.75). More generally, every solution of the phase space Einstein equations (5.66) leads to a solution of (5.75). On the other hand, not all solutions of (5.75) can be lifted to solutions of the phase space vacuum Einstein equations (5.66). Whether or not such a condition on solutions should be imposed, i.e., that the dynamics is completely determined on phase space, is presently unclear and should be ultimately prescribed by which procedure correctly matches the expectations from non-geometric string theory. We do not address further this salient point in the present paper.

Recalling our discussion from section 2.3 , it is also interesting to examine projections of the field equations (5.66) with respect to other polarisations of phase space in the $R$ flux frame. For instance, we could alternatively choose to foliate phase space with respect to constant position leaves rather than constant momentum leaves, and hence to reduce the dynamics from nonassociative phase space onto momentum space. This corresponds to embedding momentum space $\widetilde{M}$, with local coordinates $\tilde{x}_{\mu}=p_{\mu}$, in phase space via $\tilde{\sigma}: \widetilde{M} \rightarrow \mathcal{M}, \tilde{x} \mapsto \tilde{\sigma}(\tilde{x})=(0, \tilde{x})$. Correspondingly, we can restrict the classical metric to the same block diagonal form (5.68), but with the components now dependent only on momentum. By our general discussion from section 5.4 it follows that there are no $R$-flux corrections to the classical Ricci tensor on momentum space, so that momentum space geometry is uncorrected by stringy contributions; in particular, the string effective metric (5.12) coincides with the classical metric. This would appear to imply the expected result that there are no nonassociative or noncommutative corrections to the spacetime field equations in a geometric ( $H$-flux or $f$-flux) frame obtained by an $\mathrm{O}(d, d)$-rotation of the $R$-flux frame. It would be interesting to understand how this perspective ties in precisely with the possibility of Born geometry and dynamical phase space discussed in [21] using curved momentum space geometry (see [5] for further discussion of this latter point).

\subsection{First order corrections}

We will now study the vacuum Einstein equations (5.66) and (5.75) in more detail by determining the first non-trivial correction terms to the classical Einstein equations. For this, we expand the Ricci tensor from (4.83) as

$$
\begin{aligned}
\operatorname{Ric}_{B C}= & \partial_{A} \Gamma_{B C}^{A}-\partial_{C} \Gamma_{B A}^{A}+\Gamma_{B^{\prime} A}^{A} \star \Gamma_{B C}^{B^{\prime}}-\Gamma_{B^{\prime} C}^{A} \star \Gamma_{B A}^{B^{\prime}}-\mathrm{i} \kappa \mathscr{R}^{E G}{ }_{C} \Gamma_{B^{\prime} E}^{A} \star \partial_{G} \Gamma_{B A}^{B^{\prime}} \\
& +\mathrm{i} \kappa \mathscr{R}^{E G}{ }_{A}\left(\partial_{G} \partial_{C} \Gamma_{B E}^{A}-\partial_{G} \Gamma_{B^{\prime} E}^{A} \star \Gamma_{B C}^{B^{\prime}}+\partial_{G} \Gamma_{B^{\prime} C}^{A} \star \Gamma_{B E}^{B^{\prime}}+\Gamma_{B^{\prime} C}^{A} \star \partial_{G} \Gamma_{B E}^{B^{\prime}}\right) \\
& +O\left(\kappa^{2}\right) \\
= & \operatorname{Ric}_{B C}^{(0,0)}+\operatorname{Ric}_{B C}^{(0,1)}+\operatorname{Ric}_{B C}^{(1,0)}+\operatorname{Ric}_{B C}^{(1,1)}+O\left(\kappa^{2}, \hbar^{2}\right)
\end{aligned}
$$

by expanding the star-products $\star$ and using the expansion of the Levi-Civita connection from section 5.3. For the undeformed contribution we obtain the usual Ricci tensor of the classical Levi-Civita connection (5.54):

$$
\operatorname{Ric}_{B C}^{(0,0)}=\operatorname{Ric}_{B C}^{\mathrm{LC} C}:=\partial_{A} \Gamma_{B C}^{\mathrm{LC} A}-\partial_{C} \Gamma_{B A}^{\mathrm{LC} A}+\Gamma_{B^{\prime} A}^{\mathrm{LC} A} \Gamma_{B C}^{\mathrm{LC} B^{\prime}}-\Gamma_{B^{\prime} C}^{\mathrm{LC} A} \Gamma_{B A}^{\mathrm{LC} B^{\prime}}
$$


For the order $\hbar$ contribution we have

$$
\begin{aligned}
\operatorname{Ric}_{B C}^{(0,1)}= & \partial_{A} \Gamma_{B C}^{A(0,1)}-\partial_{C} \Gamma_{B A}^{A(0,1)}+\Gamma_{B^{\prime} A}^{A(0,1)} \Gamma_{B C}^{\mathrm{LC} B^{\prime}}+\Gamma_{B^{\prime} A}^{\mathrm{LC} A} \Gamma_{B C}^{B^{\prime}(0,1)} \\
& +\frac{\mathrm{i} \hbar}{2}\left(\partial_{\mu} \Gamma_{B^{\prime} A}^{\mathrm{LC} A} \tilde{\partial}^{\mu} \Gamma_{B C}^{\mathrm{LC} C B^{\prime}}-\tilde{\partial}^{\mu} \Gamma_{B^{\prime} A}^{\mathrm{L} C} \partial_{\mu} \Gamma_{B C}^{\mathrm{LC} C B^{\prime}}\right)-\Gamma_{B^{\prime} C}^{A(0,1)} \Gamma_{B A}^{\mathrm{LC} B^{\prime}} \\
& -\Gamma_{B^{\prime} C}^{\mathrm{LC} A} \Gamma_{B A}^{B^{\prime}(0,1)}-\frac{\mathrm{i} \hbar}{2}\left(\partial_{\mu} \Gamma_{B^{\prime} C}^{\mathrm{LC} A} \tilde{\partial}^{\mu} \Gamma_{B A}^{\mathrm{L} C B^{\prime}}-\tilde{\partial}^{\mu} \Gamma_{B^{\prime} C}^{\mathrm{LC} A} \partial_{\mu} \Gamma_{B A}^{\mathrm{LC} B^{\prime}}\right),
\end{aligned}
$$

where to obtain the explicit expression in terms of the classical metric tensor and LeviCivita connection one has to insert (5.63) in (5.78). Notice that $\operatorname{Ric}_{B C}^{(0,1)}$ is imaginary. Likewise, the order $\kappa$ contribution is given by

$$
\begin{aligned}
\operatorname{Ric}_{B C}^{(1,0)}= & \partial_{A} \Gamma_{B C}^{A(1,0)}-\partial_{C} \Gamma_{B A}^{A(1,0)}+\Gamma_{B^{\prime} A}^{A(1,0)} \Gamma_{B C}^{\mathrm{LC} B^{\prime}}+\Gamma_{B^{\prime} A}^{\mathrm{LC} A} \Gamma_{B C}^{B^{\prime}(1,0)} \\
& +\mathrm{i} \kappa R^{\alpha \beta \gamma} p_{\beta}\left(\partial_{\gamma} \Gamma_{B^{\prime} A}^{\mathrm{LC} A} \partial_{\alpha} \Gamma_{B C}^{\mathrm{LC} B^{\prime}}-\partial_{\gamma} \Gamma_{B^{\prime} C}^{\mathrm{LC} A} \partial_{\alpha} \Gamma_{B A}^{\mathrm{LC} B^{\prime}}\right) \\
& -\Gamma_{B^{\prime} C}^{A(1,0)} \Gamma_{B A}^{\mathrm{LC} B_{B^{\prime}}}-\Gamma_{B^{\prime} C}^{\mathrm{LC} A} \Gamma_{B A}^{B^{\prime}(1,0)}-\mathrm{i} \kappa R^{\alpha \beta \gamma} \delta_{C, \tilde{x}_{\gamma}} \Gamma_{B^{\prime} \alpha}^{\mathrm{LC} A} \partial_{\beta} \Gamma_{B A}^{\mathrm{LC} B^{\prime}} \\
& +\mathrm{i} \kappa R^{\alpha \beta \gamma} \delta_{A, \tilde{x}_{\gamma}}\left(\partial_{\beta} \partial_{C} \Gamma_{B \alpha}^{\mathrm{LC} A}-\partial_{\beta} \Gamma_{B^{\prime} \alpha}^{\mathrm{LC} A} \Gamma_{B C}^{\mathrm{LC} B^{\prime}}+\partial_{\beta} \Gamma_{B^{\prime} C}^{\mathrm{LC} A} \Gamma_{B \alpha}^{\mathrm{LC} B^{\prime}}+\Gamma_{B^{\prime} C}^{\mathrm{LC} A} \partial_{\beta} \Gamma_{B \alpha}^{\mathrm{LC} B^{\prime}}\right),
\end{aligned}
$$

where here one has to insert (5.64) to obtain the explicit expression in terms of classical quantities. Notice that $\operatorname{Ric}_{B C}^{(1,0)}$ is also imaginary.

Finally, the order $\kappa \hbar=\frac{\ell_{s}^{3}}{6}$ contribution is given by

$$
\begin{aligned}
& \operatorname{Ric}_{B C}^{(1,1)}=\partial_{A} \Gamma_{B C}^{A(1,1)}-\partial_{C} \Gamma_{B A}^{A(1,1)}+\Gamma_{B^{\prime} A}^{A(1,0)} \Gamma_{B C}^{B^{\prime}(0,1)}+\Gamma_{B^{\prime} A}^{A(0,1)} \Gamma_{B C}^{B^{\prime}(1,0)} \\
& +\Gamma_{B^{\prime} A}^{\mathrm{LC} A} \Gamma_{B C}^{B^{\prime}(1,1)}+\Gamma_{B^{\prime} A}^{A(1,1)} \Gamma_{B C}^{\mathrm{LC} C B^{\prime}}-\Gamma_{B^{\prime} C}^{A(1,0)} \Gamma_{B A}^{B^{\prime}(0,1)} \\
& -\Gamma_{B^{\prime} C}^{A(0,1)} \Gamma_{B A}^{B^{\prime}(1,0)}-\Gamma_{B^{\prime} C}^{\mathrm{L} C A} \Gamma_{B A}^{B^{\prime}(1,1)}-\Gamma_{B^{\prime} C}^{A(1,1)} \Gamma_{B A}^{\mathrm{LC} B^{\prime}} \\
& +\frac{\mathrm{i} \hbar}{2}\left(\partial_{\mu} \Gamma_{B^{\prime} A}^{A(1,0)} \tilde{\partial}^{\mu} \Gamma_{B C}^{\mathrm{LC} B^{\prime}}+\partial_{\mu} \Gamma_{B^{\prime} A}^{\mathrm{LC} A} \tilde{\partial}^{\mu} \Gamma_{B C}^{B^{\prime}(1,0)}\right. \\
& \left.-\tilde{\partial}^{\mu} \Gamma_{B^{\prime} A}^{A(1,0)} \partial_{\mu} \Gamma_{B C}^{\mathrm{LC} B^{\prime}}-\tilde{\partial}^{\mu} \Gamma_{B^{\prime} A}^{\mathrm{LC} A} \partial_{\mu} \Gamma_{B C}^{B^{\prime}(1,0)}\right) \\
& -\frac{\mathrm{i} \hbar}{2}\left(\partial_{\mu} \Gamma_{B^{\prime} C}^{A(1,0)} \tilde{\partial}^{\mu} \Gamma_{B A}^{\mathrm{LC} C B^{\prime}}+\partial_{\mu} \Gamma_{B^{\prime} C}^{\mathrm{LC} A} \tilde{\partial}^{\mu} \Gamma_{B A}^{B^{\prime}(1,0)}\right. \\
& \left.-\tilde{\partial}^{\mu} \Gamma_{B^{\prime} C}^{A(1,0)} \partial_{\mu} \Gamma_{B A}^{\mathrm{LC} B^{\prime}}-\tilde{\partial}^{\mu} \Gamma_{B^{\prime} C}^{\mathrm{LC} A} \partial_{\mu} \Gamma_{B A}^{B^{\prime}(1,0)}\right) \\
& +\mathrm{i} \kappa R^{\alpha \beta \gamma} p_{\beta}\left(\partial_{\gamma} \Gamma_{B^{\prime} A}^{A(0,1)} \partial_{\alpha} \Gamma_{B C}^{\mathrm{LC} B^{\prime}}+\partial_{\gamma} \Gamma_{B^{\prime} A}^{\mathrm{LC} A} \partial_{\alpha} \Gamma_{B C}^{B^{\prime}(0,1)}\right. \\
& \left.-\partial_{\gamma} \Gamma_{B^{\prime} C}^{A(0,1)} \partial_{\alpha} \Gamma_{B A}^{\mathrm{LC} B^{\prime}}-\partial_{\gamma} \Gamma_{B^{\prime} C}^{\mathrm{LC} A} \partial_{\alpha} \Gamma_{B A}^{B^{\prime}(0,1)}\right) \\
& -\frac{\kappa \hbar}{2} R^{\alpha \beta \gamma} p_{\beta}\left(\partial_{\mu} \partial_{\gamma} \Gamma_{B^{\prime} A}^{\mathrm{LC} A} \tilde{\partial}^{\mu} \partial_{\alpha} \Gamma_{B C}^{\mathrm{LC} B^{\prime}}-\tilde{\partial}^{\mu} \partial_{\gamma} \Gamma_{B^{\prime} A}^{\mathrm{LC} A} \partial_{\mu} \partial_{\alpha} \Gamma_{B C}^{\mathrm{LCC} B^{\prime}}\right. \\
& \left.-\partial_{\mu} \partial_{\gamma} \Gamma_{B^{\prime} C}^{\mathrm{LC} A} \tilde{\partial}^{\mu} \partial_{\alpha} \Gamma_{B A}^{\mathrm{LC} B^{\prime}}+\tilde{\partial}^{\mu} \partial_{\gamma} \Gamma_{B^{\prime} C}^{\mathrm{LC} A} \partial_{\mu} \partial_{\alpha} \Gamma_{B A}^{\mathrm{LC} B^{\prime}}\right) \\
& -\mathrm{i} \kappa R^{\alpha \beta \gamma}\left(\delta_{A, \tilde{x}_{\gamma}}\left(\partial_{\beta} \Gamma_{B^{\prime} \alpha}^{\mathrm{LC} A} \Gamma_{B C}^{B^{\prime}(0,1)}+\partial_{\beta} \Gamma_{B^{\prime} \alpha}^{A(0,1)} \Gamma_{B C}^{\mathrm{LC} B^{\prime}}\right)\right. \\
& \left.+\delta_{C, \tilde{x}_{\gamma}}\left(\Gamma_{B^{\prime} \alpha}^{\mathrm{LC} A} \partial_{\beta} \Gamma_{B A}^{B^{\prime}(0,1)}+\Gamma_{B^{\prime} \alpha}^{A(0,1)} \partial_{\beta} \Gamma_{B A}^{\mathrm{LCC} B^{\prime}}\right)\right) \\
& +\frac{\kappa \hbar}{2} R^{\alpha \beta \gamma}\left(\delta_{A, \tilde{x}_{\gamma}}\left(\partial_{\mu} \partial_{\beta} \Gamma_{B^{\prime} \alpha}^{\mathrm{LC} A} \tilde{\partial}^{\mu} \Gamma_{B C}^{\mathrm{LCC} B^{\prime}}-\tilde{\partial}^{\mu} \partial_{\beta} \Gamma_{B^{\prime} \alpha}^{\mathrm{LC} A} \partial_{\mu} \Gamma_{B C}^{\mathrm{LC} B^{\prime}}\right)\right. \\
& \left.+\delta_{C, \tilde{x}_{\gamma}}\left(\partial_{\mu} \Gamma_{B^{\prime} \alpha}^{\mathrm{LC} A} \tilde{\partial}^{\mu} \partial_{\beta} \Gamma_{B A}^{\mathrm{LC} B^{\prime}}-\tilde{\partial}^{\mu} \Gamma_{B^{\prime} \alpha}^{\mathrm{LC} A} \partial_{\mu} \partial_{\beta} \Gamma_{B A}^{\mathrm{LC} B^{\prime}}\right)\right) \\
& +\mathrm{i} \kappa R^{\alpha \beta \gamma} \delta_{A, \tilde{x}_{\gamma}}\left(\partial_{\beta} \partial_{C} \Gamma_{B \alpha}^{A(0,1)}+\partial_{\beta} \Gamma_{B^{\prime} C}^{\mathrm{LC} A} \Gamma_{B \alpha}^{B^{\prime}(0,1)}+\partial_{\beta} \Gamma_{B^{\prime} C}^{A(0,1)} \Gamma_{B \alpha}^{\mathrm{LC} B^{\prime}}\right. \\
& \left.+\Gamma_{B^{\prime} C}^{\mathrm{LC} A} \partial_{\beta} \Gamma_{B \alpha}^{B^{\prime}(0,1)}+\Gamma_{B^{\prime} C}^{A(0,1)} \partial_{\beta} \Gamma_{B \alpha}^{\mathrm{LC} B^{\prime}}\right) \\
& -\frac{\kappa \hbar}{2} R^{\alpha \beta \gamma} \delta_{A, \tilde{x}_{\gamma}}\left(\partial_{\mu} \partial_{\beta} \Gamma_{B^{\prime} C}^{\mathrm{LC} A} \tilde{\partial}^{\mu} \Gamma_{B \alpha}^{\mathrm{LC} B^{\prime}}-\tilde{\partial}^{\mu} \partial_{\beta} \Gamma_{B^{\prime} C}^{\mathrm{LC} A} \partial_{\mu} \Gamma_{B \alpha}^{\mathrm{LC} B^{\prime}}\right. \\
& \left.+\partial_{\mu} \Gamma_{B^{\prime} C}^{\mathrm{LC} A} \tilde{\partial}^{\mu} \partial_{\beta} \Gamma_{B \alpha}^{\mathrm{LC} B^{\prime}}-\tilde{\partial}^{\mu} \Gamma_{B^{\prime} C}^{\mathrm{LC} A} \partial_{\mu} \partial_{\beta} \Gamma_{B \alpha}^{\mathrm{LC} B^{\prime}}\right),
\end{aligned}
$$


where again the explicit expression in terms of the classical metric and connection is obtained after inserting (5.63)-(5.65). Like the undeformed contribution (5.77), the expression (5.80) is real.

We now consider metrics of the form (5.70) with the natural choice $\mathrm{h}^{\mu \nu}(x)=\eta^{\mu \nu}$. The pointwise inverse metric $\mathrm{g}^{M N}$ has an expansion in $\kappa$, which up to first order is given by

$$
\left(\mathrm{g}^{M N}(x)\right)=\left(\begin{array}{cc}
\mathrm{g}^{\mu \nu}(x) & -\frac{\mathrm{i} \kappa}{2} R^{\alpha \nu \gamma} \mathrm{g}^{\mu \rho} \partial_{\alpha} \mathrm{g}_{\rho \gamma} \\
-\frac{\mathrm{i} \kappa}{2} R^{\alpha \mu \gamma} \partial_{\alpha} \mathrm{g}_{\gamma \rho} \mathrm{g}^{\rho \nu} & \eta_{\mu \nu}
\end{array}\right)+O\left(\kappa^{2}\right)
$$

One caveat is that the $\kappa$-dependence of (5.70) and (5.81) will now reorder the expansion of the Levi-Civita connection in (5.62)-(5.65); for example, the classical contributions $\Gamma_{A D}^{S(0,0)}=\Gamma_{A D}^{\mathrm{LC} S}$ in $(5.62)$ will receive both type $(0,0)$ and $(1,0)$ terms. These additional contributions can be easily accounted for by using the fact that there is no momentum dependence in (5.70) and (5.81), and our results below take this reordering into account.

After summing up all expressions, the proper expansion of the connection coefficients is as follows: for the classical contribution $\Gamma_{A D}^{S(0,0)}$ the only non-zero components of the classical Levi-Civita connection in this case are

$$
\Gamma_{\mu \nu}^{\mathrm{LC} \rho}=\frac{1}{2} \mathbf{g}^{\rho \sigma}\left(\partial_{\mu} \mathrm{g}_{\sigma \nu}+\partial_{\nu} \mathrm{g}_{\mu \sigma}-\partial_{\sigma} \mathrm{g}_{\mu \nu}\right)
$$

Using (5.63) one can check that all contributions $\Gamma_{A D}^{S(0,1)}$ vanish. The only non-zero components of the corrections $\Gamma_{A D}^{S(1,0)}$ from (5.64) are

$$
\begin{aligned}
\Gamma_{\mu \nu}^{\rho(1,0)} & =-\mathrm{i} \kappa R^{\alpha \beta \gamma} p_{\beta} \mathrm{g}^{\rho \sigma}\left(\partial_{\gamma} \mathrm{g}_{\sigma \tau}\right) \partial_{\alpha} \Gamma_{\mu \nu}^{\mathrm{LC} \tau}, \\
\Gamma_{\mu \nu}^{\tilde{x}_{\rho}(1,0)} & =-\frac{\mathrm{i} \kappa}{2} R^{\alpha \rho \sigma} \mathrm{g}_{\gamma \sigma} \partial_{\alpha} \Gamma_{\mu \nu}^{\mathrm{LC} \gamma}, \\
\Gamma_{\tilde{x}_{\mu}, \nu}^{\rho(1,0)} & =\frac{\mathrm{i} \kappa}{2} R^{\alpha \mu \gamma} \mathrm{g}^{\sigma \rho} \partial_{\alpha}\left(\mathrm{g}_{\sigma \tau} \Gamma_{\gamma \nu}^{\mathrm{LC} \tau}\right), \\
\Gamma_{\mu, \tilde{x}_{\nu}}^{\rho(1,0)} & =\frac{\mathrm{i} \kappa}{2} R^{\alpha \nu \gamma} \mathrm{g}^{\rho \sigma} \partial_{\alpha}\left(\mathrm{g}_{\sigma \tau} \Gamma_{\mu \gamma}^{\mathrm{LC} \tau}\right),
\end{aligned}
$$

while the remaining correction terms $\Gamma_{A D}^{S(1,1)}$ from (5.65) have non-vanishing contributions

$$
\Gamma_{\mu \nu}^{\rho(1,1)}=\frac{\hbar \kappa}{2} R^{\alpha \beta \gamma}\left(\partial_{\alpha} \mathbf{g}^{\rho \sigma}\right)\left(\partial_{\beta} \mathbf{g}_{\sigma \tau}\right) \partial_{\gamma} \Gamma_{\mu \nu}^{\mathrm{LC} \tau}
$$

The non-zero components of the classical Ricci tensor are then

$$
\mathrm{Ric}_{\mu \nu}^{\mathrm{LC}}=\partial_{\rho} \Gamma_{\mu \nu}^{\mathrm{LC} \rho}-\partial_{\nu} \Gamma_{\mu \rho}^{\mathrm{LC} \rho}+\Gamma_{\sigma \rho}^{\mathrm{LC} \rho} \Gamma_{\mu \nu}^{\mathrm{LC} \sigma}-\Gamma_{\sigma \nu}^{\mathrm{LC} \rho} \Gamma_{\mu \rho}^{\mathrm{LC} \sigma}
$$

Although the expanded formula for $\operatorname{Ric}_{B C}$ appears to be unwieldy and very difficult to analyse in general, in this case all correction terms $\mathrm{Ric}_{B C}^{(0,1)}$ vanish, while the non-zero 
components of $\operatorname{Ric}_{B C}^{(1,0)}$ are

$$
\begin{aligned}
\operatorname{Ric}_{\mu \nu}^{(1,0)}= & \mathrm{i} \kappa R^{\alpha \beta \gamma} p_{\beta}\left(-\partial_{\rho}\left(\mathrm{g}^{\rho \sigma}\left(\partial_{\gamma} \mathrm{g}_{\sigma \tau}\right) \partial_{\alpha} \Gamma_{\mu \nu}^{\mathrm{LC} \tau}\right)+\partial_{\nu}\left(\mathrm{g}^{\rho \sigma}\left(\partial_{\gamma} \mathrm{g}_{\sigma \tau}\right) \partial_{\alpha} \Gamma_{\mu \rho}^{\mathrm{LC} \tau}\right)\right. \\
& -\Gamma_{\mu \nu}^{\mathrm{LC} \omega} \mathrm{g}^{\rho \sigma}\left(\partial_{\gamma} \mathrm{g}_{\sigma \tau}\right) \partial_{\alpha} \Gamma_{\omega \rho}^{\mathrm{LC} \tau}-\Gamma_{\rho \omega}^{\mathrm{LC} \rho} \mathrm{g}^{\omega \sigma}\left(\partial_{\gamma} \mathrm{g}_{\sigma \tau}\right) \partial_{\alpha} \Gamma_{\mu \nu}^{\mathrm{LC} \tau} \\
& +\Gamma_{\mu \rho}^{\mathrm{LC} \omega} \mathrm{g}^{\rho \sigma}\left(\partial_{\gamma} \mathrm{g}_{\sigma \tau}\right) \partial_{\alpha} \Gamma_{\omega \nu}^{\mathrm{LC} \tau}+\Gamma_{\omega \nu}^{\mathrm{LC} \rho} \mathrm{g}^{\omega \sigma}\left(\partial_{\gamma} \mathrm{g}_{\sigma \tau}\right) \partial_{\alpha} \Gamma_{\mu \rho}^{\mathrm{LC} \tau} \\
& \left.+\left(\partial_{\gamma} \Gamma_{\sigma \rho}^{\mathrm{LC} \rho}\right) \partial_{\alpha} \Gamma_{\mu \nu}^{\mathrm{LC} \sigma}-\left(\partial_{\gamma} \Gamma_{\sigma \nu}^{\mathrm{LC} \rho}\right) \partial_{\alpha} \Gamma_{\mu \rho}^{\mathrm{LC} \sigma}\right) \\
\operatorname{Ric}_{\mu, \tilde{x}_{\nu}}^{(1,0)}= & \frac{\mathrm{i} \kappa}{2} R^{\alpha \nu \gamma}\left(\partial_{\rho}\left(\mathrm{g}^{\rho \sigma} \partial_{\alpha}\left(\mathrm{g}_{\sigma \tau} \Gamma_{\gamma \mu}^{\mathrm{LC} \tau}\right)\right)+\mathrm{g}^{\rho \sigma} \Gamma_{\rho \omega}^{\mathrm{LC} \omega} \partial_{\alpha}\left(\mathrm{g}_{\sigma \tau} \Gamma_{\gamma \mu}^{\mathrm{LC} \tau}\right)\right. \\
& \left.-\mathrm{g}^{\rho \sigma} \Gamma_{\mu \rho}^{\mathrm{LC} \omega} \partial_{\alpha}\left(\mathrm{g}_{\sigma \tau} \Gamma_{\gamma \omega}^{\mathrm{LC} \tau}\right)+2 \Gamma_{\sigma \alpha}^{\mathrm{LC} \rho} \partial_{\gamma} \Gamma_{\mu \rho}^{\mathrm{LC} \sigma}\right) \\
\operatorname{Ric}_{\tilde{x}_{\mu}, \nu}^{(1,0)}= & \frac{\mathrm{i} \kappa}{2} R^{\alpha \mu \gamma}\left(\partial_{\rho}\left(\mathrm{g}^{\rho \sigma} \partial_{\alpha}\left(\mathrm{g}_{\sigma \tau} \Gamma_{\gamma \nu}^{\mathrm{LC} \tau}\right)\right)-\partial_{\nu}\left(\mathrm{g}^{\rho \sigma} \partial_{\alpha}\left(\mathrm{g}_{\sigma \tau} \Gamma_{\gamma \rho}^{\mathrm{LC} \tau}\right)\right)\right. \\
& \left.+\mathrm{g}^{\rho \sigma} \Gamma_{\rho \omega}^{\mathrm{LC} \omega} \partial_{\alpha}\left(\mathrm{g}_{\sigma \tau} \Gamma_{\gamma \nu}^{\mathrm{LC} \tau}\right)-\mathrm{g}^{\rho \sigma} \Gamma_{\rho \nu}^{\mathrm{LC} \omega} \partial_{\alpha}\left(\mathrm{g}_{\sigma \tau} \Gamma_{\gamma \omega}^{\mathrm{LC} \tau}\right)\right) .
\end{aligned}
$$

Notice that all terms of type $(1,0)$ are imaginary. Finally, the only non-zero components of $\operatorname{Ric}_{B C}^{(1,1)}$ are given by

$$
\begin{aligned}
\operatorname{Ric}_{\mu \nu}^{(1,1)}= & \frac{\hbar \kappa}{2} R^{\alpha \beta \gamma}\left(\partial_{\rho}\left(\partial_{\alpha} \mathrm{g}^{\rho \sigma}\left(\partial_{\beta} \mathrm{g}_{\sigma \tau}\right) \partial_{\gamma} \Gamma_{\mu \nu}^{\mathrm{LC} \tau}\right)-\partial_{\nu}\left(\partial_{\alpha} \mathrm{g}^{\rho \sigma}\left(\partial_{\beta} \mathrm{g}_{\sigma \tau}\right) \partial_{\gamma} \Gamma_{\mu \rho}^{\mathrm{LC} \tau}\right)\right. \\
& +\partial_{\gamma} \mathrm{g}_{\tau \omega}\left(\partial_{\alpha}\left(\mathrm{g}^{\sigma \tau} \Gamma_{\sigma \nu}^{\mathrm{LC} \rho}\right) \partial_{\beta} \Gamma_{\mu \rho}^{\mathrm{LC} \omega}-\partial_{\alpha}\left(\mathrm{g}^{\sigma \tau} \Gamma_{\sigma \rho}^{\mathrm{LC} \rho}\right) \partial_{\beta} \Gamma_{\mu \nu}^{\mathrm{LC} \omega}\right. \\
& +\left(\Gamma_{\mu \rho}^{\mathrm{LC} \sigma} \partial_{\alpha} \mathrm{g}^{\rho \tau}-\partial_{\alpha} \Gamma_{\mu \rho}^{\mathrm{LC} \sigma} \mathrm{g}^{\rho \tau}\right) \partial_{\beta} \Gamma_{\sigma \nu}^{\mathrm{LC} \omega} \\
& \left.\left.-\left(\Gamma_{\mu \nu}^{\mathrm{LC} \sigma} \partial_{\alpha} \mathrm{g}^{\rho \tau}-\partial_{\alpha} \Gamma_{\mu \nu}^{\mathrm{LC} \sigma} \mathrm{g}^{\rho \tau}\right) \partial_{\beta} \Gamma_{\sigma \rho}^{\mathrm{LC} \omega}\right)\right)
\end{aligned}
$$

Now we apply the reduction described in section 5.5, and altogether we find for the Ricci tensor $\mathrm{Ric}_{\mu \nu}^{\circ}$ on spacetime $M$ up to first order:

$$
\begin{aligned}
\operatorname{Ric}_{\mu \nu}^{\circ}= & \operatorname{Ric}_{\mu \nu}^{\mathrm{LC}}+\frac{\ell_{s}^{3}}{12} R^{\alpha \beta \gamma}\left(\partial_{\rho}\left(\partial_{\alpha} \mathrm{g}^{\rho \sigma}\left(\partial_{\beta} \mathrm{g}_{\sigma \tau}\right) \partial_{\gamma} \Gamma_{\mu \nu}^{\mathrm{LC} \tau}\right)-\partial_{\nu}\left(\partial_{\alpha} \mathrm{g}^{\rho \sigma}\left(\partial_{\beta} \mathrm{g}_{\sigma \tau}\right) \partial_{\gamma} \Gamma_{\mu \rho}^{\mathrm{LC} \tau}\right)\right. \\
& +\partial_{\gamma} \mathrm{g}_{\tau \omega}\left(\partial_{\alpha}\left(\mathrm{g}^{\sigma \tau} \Gamma_{\sigma \nu}^{\mathrm{LC} \rho}\right) \partial_{\beta} \Gamma_{\mu \rho}^{\mathrm{LC} \omega}-\partial_{\alpha}\left(\mathrm{g}^{\sigma \tau} \Gamma_{\sigma \rho}^{\mathrm{LC} \rho}\right) \partial_{\beta} \Gamma_{\mu \nu}^{\mathrm{LC} \omega}\right. \\
& +\left(\Gamma_{\mu \rho}^{\mathrm{LC} \sigma} \partial_{\alpha} \mathrm{g}^{\rho \tau}-\partial_{\alpha} \Gamma_{\mu \rho}^{\mathrm{LC} \sigma} \mathrm{g}^{\rho \tau}\right) \partial_{\beta} \Gamma_{\sigma \nu}^{\mathrm{LC} \omega} \\
& \left.\left.-\left(\Gamma_{\mu \nu}^{\mathrm{LC} \sigma} \partial_{\alpha} \mathrm{g}^{\rho \tau}-\partial_{\alpha} \Gamma_{\mu \nu}^{\mathrm{LC} \sigma} \mathrm{g}^{\rho \tau}\right) \partial_{\beta} \Gamma_{\sigma \rho}^{\mathrm{LC} \omega}\right)\right)
\end{aligned}
$$

for $\mu, \nu=1, \ldots, d$. One readily checks that the linear $R$-flux correction to the classical Ricci tensor is not a total derivative: while the first two lines of the correction in (5.90) are total derivatives, the last two lines are not. The consistent reduction to spacetime has thus achieved two remarkable and desirable features: not only do we find that the nonassociative $R$-flux gravitational corrections lead to non-trivial dynamical consequences on spacetime, but they are also independent of $\hbar$ and real-valued, in contrast to what happens in the usual metric formulations of noncommutative gravity [7]. Notice that this latter feature in itself singles out the zero momentum leaf among all constant momentum leaves: pulling back to a leaf of constant momentum $p=p^{\circ}$ generally gives a non-vanishing imaginary contribution $\left.\operatorname{Ric}_{\mu \nu}^{(1,0)}\right|_{p=p^{\circ}}$ from (5.86) to the spacetime Ricci tensor (5.90); indeed, 
in that case the pullback via the phase space star-product $\star$ yields associative Moyal-Weyl star-product deformations of the usual closed string scattering amplitudes with constant bivector $\theta^{\alpha \beta}=2 \kappa R^{\alpha \beta \gamma} p_{\gamma}^{\circ}[5]$, and $\left.\operatorname{Ric}_{\mu \nu}^{(1,0)}\right|_{p=p^{\circ}}$ coincides with the first order contribution to the noncommutative Ricci tensor from [7]. The potential physical significance of the $p^{\circ} \neq 0$ leaves is discussed in [5].

It would be interesting to confirm explicitly that these features all persist to higher orders, and to find explicit solutions of the spacetime vacuum equations (5.75) for nonassociative gravity. Note that these equations are linear in the $R$-flux, whereas the $H$-flux modified Einstein equations at leading order in string worldsheet perturbation theory and for constant dilaton read $\mathrm{Ric}_{\mu \nu}^{\mathrm{LC}}=\frac{1}{4} H_{\mu \alpha \beta} H_{\nu}{ }^{\alpha \beta}$ which by T-duality would naively imply that the leading corrections should be of quadratic order in the $R$-flux. Here the first non-trivial contribution to the spacetime curvature tensor is of order $O(\kappa \hbar)$ which is the order in which the first nonassociative contributions appear. As this is a second order contribution when one expands the twist element (2.3), it is natural that the curvature (and torsion) have corrections at this order.

\section{Conclusions}

In this paper we have provided and developed a formalism leading to a consistent approach to nonassociative gravity induced by locally non-geometric constant $R$-flux backgrounds of string theory in the parabolic phase space model of [25]. The construction relied on the proper characterization of tensor fields in nonassociative geometry as well as their covariance under the quasi-Hopf algebra generated by infinitesimal diffeomorphisms on twisted nonassociative phase space. The unique Levi-Civita connection of any metric g has been determined at all orders in the nonassociative deformation parameters. The vacuum Einstein equations have been obtained also at all orders, and the first order corrections to the classical equations explicitly calculated, which is the order at which the corresponding string theory calculations are reliable.

We have then pulled back the vacuum Einstein equations on phase space $\mathcal{M}$ to spacetime $M$ via the zero momentum section $\sigma: M \rightarrow \mathcal{M}$. General covariance of these latter equations is on the one hand guaranteed by the geometric pullback operation. On the other hand, it could be studied explicitly by considering the projection of the quantum Lie algebra of nonassociative diffeomorphisms from section 3.7 to the zero momentum leaf, as pursued in [5], where it was illustrated how nonassociativity survives in the action of diffeomorphisms on spacetime. Ultimately, these symmetries should be compared to the classical diffeomorphism symmetries of closed string theory and to the generalised diffeomorphism symmetries of double field theory.

Further insights into this nonassociative theory of gravity on spacetime should be obtained by studying the pullbacks to spacetime also of the torsion and the Riemann curvature tensors. Additional investigations relating the curved phase space geometry to the curved spacetime geometry, and in particular the other possible spacetime geometries obtained by considering different foliations of the manifold $\mathcal{M}$, and not only those defined by constant momentum leaves and constant position leaves, are left for future work. These investi- 
gations, and the construction of a dynamical action principle for nonassociative gravity, should clarify the expected relevance in the contexts of closed string theory and double field theory of the field equations we have obtained, and in particular their interpretations as low-energy effective field equations of closed string theory.

\section{Acknowledgments}

We thank Ralph Blumenhagen, Leonardo Castellani, Michael Fuchs, Chris Hull, Dieter Lüst, Emanuel Malek, Eric Plauschinn, Alexander Schenkel and Peter Schupp for helpful discussions. This work was supported in part by the Action MP1405 QSPACE from the European Cooperation in Science and Technology (COST). The work of P.A. and R.J.S. was supported in part by the Research Support Fund of the Edinburgh Mathematical Society, and by the Consolidated Grant ST/L000334/1 from the U.K. Science and Technology Facilities Council. The work of P.A. is partially supported by INFN, CSN4, Iniziativa Specifica GSS. P.A. is also affiliated to INdAM, GNFM (Istituto Nazionale di Alta Matematica, Gruppo Nazionale di Fisica Matematica). The work of M.D.C. is supported by Project ON171031 of the Serbian Ministry of Education and Science.

Open Access. This article is distributed under the terms of the Creative Commons Attribution License (CC-BY 4.0), which permits any use, distribution and reproduction in any medium, provided the original author(s) and source are credited.

\section{References}

[1] D. Andriot, M. Larfors, D. Lüst and P. Patalong, (Non-)commutative closed string on T-dual toroidal backgrounds, JHEP 06 (2013) 021 [arXiv:1211.6437] [INSPIRE].

[2] P. Aschieri, Noncommutative Riemannian geometry and gravity, in preparation.

[3] P. Aschieri and L. Castellani, Noncommutative gravity solutions,

J. Geom. Phys. 60 (2010) 375 [arXiv:0906.2774] [InSPIRE].

[4] P. Aschieri and A. Schenkel, Noncommutative connections on bimodules and Drinfeld twist deformation, Adv. Theor. Math. Phys. 18 (2014) 513 [arXiv:1210.0241] [INSPIRE].

[5] P. Aschieri and R.J. Szabo, Triproducts, nonassociative star products and geometry of R-flux string compactifications, J. Phys. Conf. Ser. 634 (2015) 012004 [arXiv:1504.03915] [INSPIRE].

[6] P. Aschieri, M. Dimitrijević, F. Meyer and J. Wess, Noncommutative geometry and gravity, Class. Quant. Grav. 23 (2006) 1883 [hep-th/0510059] [INSPIRE].

[7] P. Aschieri, C. Blohmann, M. Dimitrijević, F. Meyer, P. Schupp and J. Wess, A Gravity theory on noncommutative spaces, Class. Quant. Grav. 22 (2005) 3511 [hep-th/0504183] [INSPIRE].

[8] I. Bakas and D. Lüst, 3-cocycles, non-associative star-products and the magnetic paradigm of R-flux string vacua, JHEP 01 (2014) 171 [arXiv:1309.3172] [INSPIRE].

[9] I. Bakas and D. Lüst, T-duality, quotients and currents for non-geometric closed strings, Fortsch. Phys. 63 (2015) 543 [arXiv:1505.04004] [INSPIRE]. 
[10] G.E. Barnes, A. Schenkel and R.J. Szabo, Nonassociative geometry in quasi-Hopf representation categories I: Bimodules and their internal homomorphisms,

J. Geom. Phys. 89 (2014) 111 [arXiv:1409.6331] [InSPIRE].

[11] G.E. Barnes, A. Schenkel and R.J. Szabo, Nonassociative geometry in quasi-Hopf representation categories II: Connections and curvature, J. Geom. Phys. 106 (2016) 234 [arXiv: 1507.02792] [INSPIRE].

[12] G.E. Barnes, A. Schenkel and R.J. Szabo, Working with nonassociative geometry and field theory, PoS (CORFU2015) 081 [arXiv: 1601.07353] [INSPIRE].

[13] C.D.A. Blair, Non-commutativity and non-associativity of the doubled string in non-geometric backgrounds, JHEP 06 (2015) 091 [arXiv: 1405.2283] [INSPIRE].

[14] R. Blumenhagen and M. Fuchs, Towards a theory of nonassociative gravity, JHEP 07 (2016) 019 [arXiv: 1604.03253] [INSPIRE].

[15] R. Blumenhagen and E. Plauschinn, Nonassociative gravity in string theory?, J. Phys. A 44 (2011) 015401 [arXiv:1010.1263] [inSPIRE].

[16] R. Blumenhagen, A. Deser, D. Lüst, E. Plauschinn and F. Rennecke, Non-geometric fluxes, asymmetric strings and nonassociative geometry, J. Phys. A 44 (2011) 385401 [arXiv:1106.0316] [INSPIRE].

[17] R. Blumenhagen, M. Fuchs, F. Haßler, D. Lüst and R. Sun, Non-associative deformations of geometry in double field theory, JHEP 04 (2014) 141 [arXiv: 1312.0719] [INSPIRE].

[18] M. Bojowald, S. Brahma, U.Büyükçam and T. Strobl, Monopole star products are non-alternative, JHEP 04 (2017) 028 [arXiv: 1610.08359] [INSPIRE].

[19] C. Condeescu, I. Florakis and D. Lüst, Asymmetric orbifolds, non-geometric fluxes and non-commutativity in closed string theory, JHEP 04 (2012) 121 [arXiv:1202.6366] [INSPIRE].

[20] V.G. Drinfeld, Quasi-Hopf algebras, Alg. Anal. 1N6 (1989) 114.

[21] L. Freidel, R.G. Leigh and D. Minic, Born reciprocity in string theory and the nature of spacetime, Phys. Lett. B 730 (2014) 302 [arXiv:1307.7080] [InSPIRE].

[22] C. Kassel, Quantum Groups, Springer, New York U.S.A. (1995).

[23] V.G. Kupriyanov and R.J. Szabo, $G_{2}$-structures and quantization of non-geometric M-theory backgrounds, JHEP 02 (2017) 099 [arXiv: 1701.02574] [INSPIRE].

[24] V.G. Kupriyanov and D.V. Vassilevich, Nonassociative Weyl star products, JHEP 09 (2015) 103 [arXiv: 1506.02329] [INSPIRE].

[25] D. Lüst, T-duality and closed string non-commutative (doubled) geometry, JHEP 12 (2010) 084 [arXiv:1010.1361] [INSPIRE].

[26] D. Mylonas, P. Schupp and R.J. Szabo, Membrane $\sigma$-models and quantization of non-geometric flux backgrounds, JHEP 09 (2012) 012 [arXiv:1207.0926] [INSPIRE].

[27] D. Mylonas, P. Schupp and R.J. Szabo, Non-geometric fluxes, quasi-Hopf twist deformations and nonassociative quantum mechanics, J. Math. Phys. 55 (2014) 122301 [arXiv:1312.1621] [INSPIRE].

[28] R.D. Schafer, An Introduction to Nonassociative Algebras, Dover Publications, New York U.S.A. (1995). 